EPJ manuscript No.

(will be inserted by the editor)

\title{
JETS AND QCD: A Historical Review of the Discovery of the Quark and Gluon Jets and its Impact on $Q C D^{\star}$
}

\author{
A.Ali ${ }^{1}$ and G.Kramer ${ }^{2}$ \\ 1 DESY, D-22603 Hamburg (Germany) \\ 2 Universität Hamburg, D-22761 Hamburg (Germany)
}

\begin{abstract}
The observation of quark and gluon jets has played a crucial role in establishing Quantum Chromodynamics [QCD] as the theory of the strong interactions within the Standard Model of particle physics. The jets, narrowly collimated bundles of hadrons, reflect configurations of quarks and gluons at short distances. Thus, by analysing energy and angular distributions of the jets experimentally, the properties of the basic constituents of matter and the strong forces acting between them can be explored. In this review, which is primarily a description of the discovery of the quark and gluon jets and the impact of their observation on Quantum Chromodynamics, we elaborate, in particular, the role of the gluons as the carriers of the strong force. Focusing on these basic points, jets in $e^{+} e^{-}$collisions will be in the foreground of the discussion and we will concentrate on the theory that was contemporary with the relevant experiments at the electron-positron colliders. In addition we will delineate the role of jets as tools for exploring other particle aspects in $e p$ and $p p / p \bar{p}$ collisions - quark and gluon densities in protons, measurements of the QCD coupling, fundamental 2-2 quark/gluon scattering processes, but also the impact of jet decays of top quarks, and $W^{ \pm}, Z$ bosons on the electroweak sector. The presentation to a large extent is formulated in a non-technical language with the intent to recall the significant steps historically and convey the significance of this field also to communities beyond high energy physics.
\end{abstract}

\section{Introduction}

Quantum Chromodynamics [QCD], the theory of the strong interactions within the Standard Model of particle physics 112/314, describes the building blocks of strongly interacting particles, like proton, neutron and many others, and the forces acting between them. The fundamental building blocks of these particles are the spin- $1 / 2$ quarks $q$ [5]6, which come in three families. Their masses cover a large range 7]. The three lightest quarks $u, d, s$ weigh only a small fraction of the proton mass, the charm quark $c$ just about the proton mass while the two heavy quarks $b, t$ weigh more

\footnotetext{
* In Memoriam: Hans Joos
} 
than 5 and 180 times the proton mass, respectively. Baryons, like proton and neutron, are composed of three quarks $q q q$, while mesons, like pions, are bound states $q \bar{q}$ of quark-antiquark pairs.

Since the spin and the spatial $S$-wave functions of the lightest baryons are symmetric under the exchange of quarks, the Pauli principle demands the quarks to be labelled by new charges, called colours, which discriminate between the three components of the baryons 89910 . Rephrased within the $\mathrm{SU}(3)_{C}$ symmetry group for three colour degrees of freedom, the colour wave function is antisymmetric. This threefold antisymmetric combination of colours renders baryons non-coloured, i.e. they are white $\mathrm{SU}(3)_{C}$ singlets; summing up the quark colours symmetrically in mesons, these hadrons are white too. By reducing the lifetime of the neutral pion by a factor $3^{2}=9$, the three-colour extension reconciles the prediction of the quark model with the experimental measurement, a crucial point in establishing the colour charges.

Equivalently to the electric charges in electrodynamics, the colour charges of the quarks can serve as sources for force fields, which bind the quarks within the mesons and baryons [11. Eight such gluon fields $g$ are predicted by the non-abelian gauge group $\mathrm{SU}(3)_{C}$. Like the photon field they are vector fields with spin $=1$, but in contrast to the photon they carry colour charges themselves, mediating colour flips of quarks by absorption or emission. This theory, Quantum Chromodynamics, is theoretically described by a non-abelian Yang-Mills gauge theory 12. Gluons couple to each other, giving rise to three- and four-gluon interactions. These selfinteractions of the gluons have profound consequences for the QCD coupling. While virtual fermionic quarks render the vacuum colour-diamagnetic, the large contribution of virtual bosonic gluons renders the vacuum finally colour-paramagnetic. Thus, in contrast to the electric coupling, the QCD coupling decreases with decreasing distance and the quarks and gluons become asymptotically free [3/4]. Quarks and gluons therefore interact weakly at short distances while the strength of their interactions grows with increasing distance, suggesting the permanent confinement of particles carrying non-zero colour charges 13 .

Quarks can be seen in the scattering of electrons or neutrinos off nucleons. The final-state pattern of these processes reveals that the leptons scatter off point-like, nearly massless spin- $1 / 2$ constituents of the nucleons which carry the electric and weak charges of the quarks. Gluons inside nucleons, which do not carry electric nor weak charges, manifest themselves only indirectly. Half of the momentum of fast moving nucleons cannot be accounted for by the spectrum of the quarks alone, and it must be attributed to gluons as flavour-neutral constituents [14. In addition, the quark spectrum is modified by gluon bremsstrahlung if the energy of the impinging leptons is raised from low to high values 15 .

However, QCD suggests another method to unravel its basic constituents. As a result of asymptotic freedom, quarks and gluons move as quasi-free particles, called partons [16, at short distances. When these coloured objects are accelerated in scattering processes, they develop bremsstrahlung cascades of narrowly collimated gluons and quark-antiquark pairs, which finally transform to equally well collimated hadrons at distances at the colour confinement radius of about $1 \mathrm{fm}\left[10^{-13} \mathrm{~cm}\right]$. Thus, the quarks and gluons at small distances map themselves into jets of hadrons at large distances. Since the quanta associated with the confinement forces are soft, their impact on the energies and momenta of the jets is small so that the configurations of high-energy quarks and gluons at short distances are truly reflected in the energy and angular distributions of the jets. Since these jets can be observed experimentally, the properties of quarks and gluons can be determined experimentally by jet analyses, such as their spins, flavour and colour charges, and their basic interactions.

It should be stressed here that the field of jet physics and QCD owes a great deal of gratitude to the development and successful operations of high energy colliders, in 
particular, electron-positron colliders. Starting from SPEAR at SLAC, which started the physics runs in 1972 and had a maximum beam energy of $4 \mathrm{GeV}$, the subsequently built $e^{+} e^{-}$colliders DORIS (physics start 1973; maximum beam energy $5.6 \mathrm{GeV}$ ) and PETRA (physics start 1978; maximum beam energy $23.4 \mathrm{GeV}$ ) at DESY, PEP (physics start 1980; maximum beam energy $15 \mathrm{GeV}$ ) and SLC (physics start 1989; maximum beam energy $50 \mathrm{GeV}$ ) at SLAC, TRISTAN at KEK (physics start 1987; maximum beam energy $32 \mathrm{GeV}$ ), and LEP (physics start 1989; maximum beam energy 104.6 GeV) at CERN, saw the main jet activity and detailed tests of QCD. The results from these machines are the primary focus of this review and are discussed in the first six chapters. However, in a long epilogue, described in chapter 7, entitled jets as tools, we have discussed some selected results related to QCD and jets, which have come out from the electron-proton collider HERA (physics start 1992; maximum $e^{+} / e^{-}$beam energy $27.6 \mathrm{GeV}$ and maximum proton energy $920 \mathrm{GeV}$ ) at DESY, and the hadron colliders Tevatron (physics start 1987; maximum $p$ and $\bar{p}$ energy $980 \mathrm{GeV}$ ) at Fermilab and finally the LHC (physics start 2010; maximum proton beam energy so far $3.5 \mathrm{TeV}$ ). It is not our mandate to discuss the technical aspects of these machines, which will take us too far afield from the main focus, namely historical development of jets and QCD from the theoretical and experimental points of view. For the interested readership of this review, the high energy machine related aspects are summarised concisely in Reviews of Particle Physics by the Particle Data Group (PDG). Many of these colliders, in fact all the $e^{+} e^{-}$colliders, are no longer working in particle physics, and for these we refer to the 1996 PDG review [17], while for the others to the 2010 PDG review [7.

Quite early, the final states in $e^{+} e^{-}$annihilation to hadrons had been predicted to consist [primarily] of two jets evolving from a quark-antiquark pair produced in the collision process 181920 :

$$
e^{+} e^{-} \rightarrow q \bar{q} \rightarrow 2 \text { jets }
$$

Experimental evidence for these quark jets was first provided at the $e^{+} e^{-}$collider SPEAR [21|22] by demonstrating that the hadrons in the final states were not isotropically distributed but accumulated near the central event axis specified by the momenta of the quarks [23. At PETRA energies $(12 \leq \sqrt{s} \leq 46.6 \mathrm{GeV})$ the two jets could be recognised without any sophisticated analysis, $c f$. Fig 1 (left-side frame). Angular distributions and charge analyses finally proved the jets to be associated with spin-1/2 quarks indeed.

First indirect evidence of gluons was provided by the PLUTO collaboration at the $e^{+} e^{-}$collider DORIS [24] from the decay $\Upsilon(1 S) \rightarrow g g g$. However, as $\Upsilon(1 S)$ has a mass of $9.46 \mathrm{GeV}$, significant non-perturbative contributions had to be taken into account. PLUTO used their 2-jet data below the resonance to extract the $q^{*} \rightarrow$ hadrons fragmentation and used this to estimate also the fragmentation $g^{*} \rightarrow$ hadrons. With this, their analysis was in agreement with the expectations from the underlying process $\Upsilon(1 S) \rightarrow g g g$. Gluon jets were later discovered unambiguously at the $e^{+} e^{-}$collider PETRA 25]26 27/28] running at higher energy (typically $30 \mathrm{GeV}$ ). A 3-jet event from the very early PETRA data 29 is shown in Fig. 1](right-side frame). Such events had been predicted theoretically [30] for configurations in which the quark pair produced in $e^{+} e^{-}$annihilation radiates a hard non-collinear gluon:

$$
e^{+} e^{-} \rightarrow q \bar{q} g \rightarrow 3 \text { jets }
$$

This bremsstrahlung mechanism is characteristic for gauge theories and it is familiar from electrodynamics where charges accelerated in collision processes emit photons, as in electron-positron scattering $e^{+} e^{-} \rightarrow e^{+} e^{-} \gamma$, for example. Bremsstrahlung gluons in QCD which transform to hadron jets generate characteristic patterns in the 


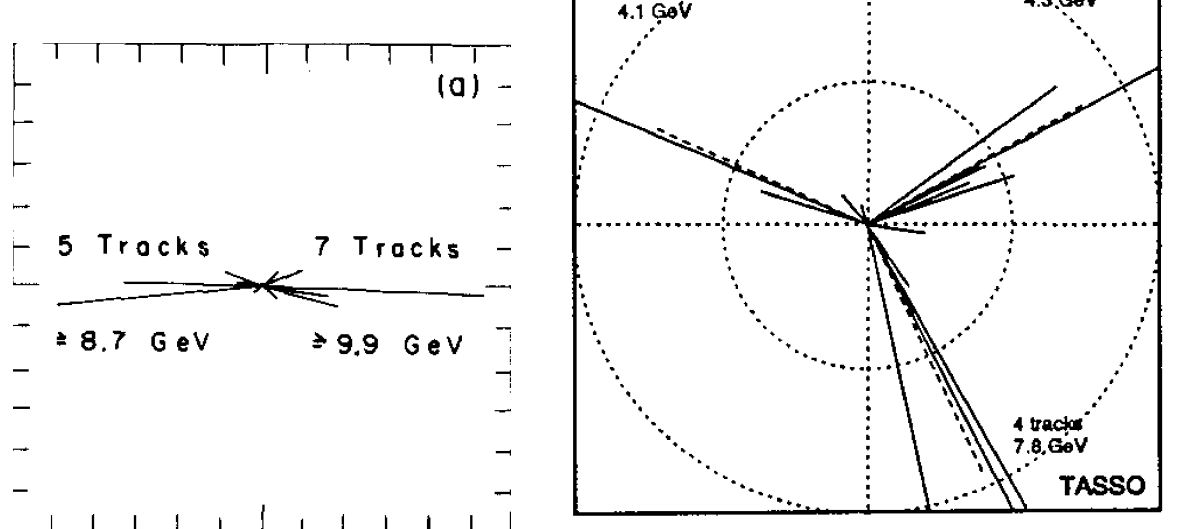

Fig. 1. Observation of (a) 2-jet final states in electron-positron annihilation to hadrons: $e^{+} e^{-} \rightarrow q \bar{q} \rightarrow 2$ jets (TASSO 25); and (b) 3-jet final states in gluon bremsstrahlung off quarks in $e^{+} e^{-}$annihilation: $e^{+} e^{-} \rightarrow q \bar{q} g \rightarrow 3$ jets in the TASSO detector [29].

final states which allow one to prove the existence of gluons: With increasing energy the primary quark jets become narrower; the events become flat and "Mercedes-Starlike" ( $Y$-shaped); and finally three well separated jets emerge. Detailed comparison of the event structure with the underlying theory (QCD) required apart from the perturbative (hard) processes also modeling of the non-perturbative (soft) features of the quark and gluon fragmentation. Hence, event generators, incorporating the perturbative and non-perturbative aspects of QCD were necessary to relate the emerging jet distributions to the predictions derived from gluon bremsstrahlung in QCD. References 31.32 illustrate the early use of such event generators, taking the form of Monte Carlo simulations to match the theretical calculations with the experimental measurements, which were state-of-the-art tools at that time, and which helped in establishing the properties of the quark and gluon jets. To avoid any confusion, Monte Carlo in the present context is a numerical computational technique to calculate multi-dimensional distributions of a process in which events are generated randomly but weighted to reflect the underlying dynamics. Dedicated experiments at PETRA and PEP and theoretical progress in the 80's greatly consolidated jet physics and led to quantitative tests of QCD. A more modern view of the use of Monte Carlo programs, in particular, their role as tools in hard hadronic collisions can be found in recent reviews, for example 33 .

The program to establish QCD in studying quark and gluon jets was naturally continued at the $e^{+} e^{-}$collider LEP, see, for example, 34], where the increased energy could be exploited to measure the gluon self-interactions in multijet events,

$$
e^{+} e^{-} \rightarrow q \bar{q} q^{\prime} \bar{q}^{\prime}, \quad q \bar{q} g g \rightarrow 4 j e t s,
$$

with the production amplitudes dominated by the $q \bar{q} g g$ states, which included the virtual gluon splitting, e.g. $g^{*} \rightarrow g g$. By measuring energy and angular distributions of these 4jet-events the colour charge of gluons could be determined, the crucial element for generating asymptotic freedom in QCD. Correspondingly, the variation of the quark/gluon coupling could be examined for a large range of energies, though experiments at PEP, PETRA and TRISTAN had already confirmed the running of $\alpha_{s}\left(Q^{2}\right)$ in agreement with the renormalisation group $(\mathrm{RG})$ equation. 
The quark/gluon jet phenomena were also indicated at the $p p$ collider ISR 35], before high-energy jets were unambiguously isolated at the $S p \bar{p} S$ [36]. Since then, jets in hadronic collisions have become precision tools in not only testing QCD and the electroweak physics at the highest available energies (such as at the Tevatron and the LHC), but also in searching for phenomena beyond-the-Standard-Model (BSM), such as dijet resonances and quark substructure. By the same token, jet phenomena observed at hadron-hadron and lepton-hadron colliders have provided fundamental information on the quark and gluon densities (parton distribution functions) of the proton. We shall review this towards the end of this paper, but for now concentrate on the general development of jet physics and QCD which took place in the context of $e^{+} e^{-}$colliders.

Quark and gluon processes at short distances can be treated, due to asymptotic freedom of QCD, in perturbative expansions for the weakly interacting fields. Therefore the basic short-distance processes as well as the evolution of the quark/gluon cascades are well controlled theoretically. However, the final transition from the quark/gluon configurations to hadrons is a long-distance process governed by large values of the QCD coupling which cannot be treated in perturbation theory and which, so far, cannot be analysed rigorously. Instead, QCD-inspired models have been developed which parametrise the transition phenomenologically. Two alternative approaches have been worked out in detail. In the first picture a quark moving out of the short-distance regime generates a string-like gluonic flux tube which breaks up repeatedly by spontaneous quark-antiquark creation when its length approaches about $1 \mathrm{fm}$. This mechanism generates a jet of collimated hadrons with energy and direction corresponding to the initial high-energy quark 37. Gluons had been treated analogously 3132, or they were assumed to generate kinks, local depositions of energy and momentum in the strings stretched between quarks and antiquarks [38. Alternatively in cluster fragmentation, after splitting all final gluons in a quark/gluon cascade to quarks and antiquarks, $q \bar{q}$ pairs with low invariant masses transform to hadronic resonances which eventually decay to the standard low-mass mesons and baryons [39].

After the important work of Ref. [40] (see, also [414243]), numerous methods have been proposed, with steadily increasing refinement, to reconstruct the jets experimentally. One class consists of algorithms based on sequential jet recombination. Particles are sequentially combined if their distance in momentum space falls below a pre-set minimum. Typical examples are the JADE algorithm [44, where the distance is defined by the invariant mass of pairs, developed later to algorithms based on transverse momenta $k_{t}$. A second class is built by cone algorithms in which particles belonging to pre-defined cones are grouped into jets. Originally introduced to regulate singularities in infrared and collinear quark-gluon configurations [4546], they have been developed to a standard method in hadron collider analyses.

The original jet analyses at PETRA were based on independent-jet fragmentation [31/32, providing a valid tool for reconstructing the quark/gluon configurations at small distances in $e^{+} e^{-}$annihilation. Subtle effects observed later in the hadron distributions between jets, were interpreted as string effect 3847], or explained alternatively by additional soft gluon radiation with angular ordering [4]. PYTHIA 4950, HERWIG 51] and SHERPA [52] are modern versions of Monte Carlo programs which are used in present jet analyses.

The connection of jets with QCD has been extensively treated in the literature under theoretical and experimental aspects, see e.g. [53. [55. This review will summarise the basic concepts of jet physics, intended to describe how jet physics has been exploited to establish QCD as the non-abelian quark/gluon gauge field theory of the strong interactions. Addressing also communities outside high energy physics, the review is presented mostly in a non-technical language, giving a qualitative account of theoretical and experimental developments which have dramatically changed 
the earlier picture of the strong forces in particle physics. In doing this, we have included some landmark measurements in a chronological order as they were reported. The same remark applies to the discussion of the theoretical aspects, and we have emphasized only works which were contemporary with the discoveries. The picture now is based on a few fundamental principles summarised succinctly in Quantum Chromodynamics.

The topics on which we concentrate are the non-perturbative and perturbative elements of quark/gluon jets, including experimental and phenomenological methods to define the jets. Early evidence and indirect indications of quark and gluon jets in $e^{+} e^{-}$annihilation to quarks at SPEAR and $\Upsilon$ decays to gluons at DORIS will be reviewed. In the central core of this paper, we will describe the theoretical basis of the discovery of gluons in the three-jet events at PETRA and the measurement of their properties. The picture will be completed with LEP. Finally we will demonstrate in a few examples how jets can be used as tools for measuring other parameters and fundamental processes of QCD, the gluon content of nucleons, QCD Rutherford scattering, etc., but also how to exploit jets for identifying electroweak $W, Z$ and Higgs bosons, top-quark physics, and search for new phenomena, in particular possible substructure of partons. Such problems have been addressed at HERA and the Tevatron, and they will play an important role at the LHC. However, despite discussing some of the most recent measurements in jets and QCD, this is not a review of the up-to-date theoretical advances. We have included some of these topics to introduce the readers to the vast areas of particle physics research in which jets and QCD have branched out, but emphasize that this article aims primarily at providing a historical perspective.

This paper is organised in 8 sections and the main topics discussed are as follows: Fragmentation properties of quarks and gluons (section 2), discovery of quark jets at SPEAR and the first application of perturbative QCD to derive the 2-jet cross section in $e^{+} e^{-}$annihilation (section 3 ), gluon jets in $\Upsilon$ decays and the basic partonic process $\Upsilon \rightarrow g g g$ (section 4), jets in QCD and at PETRA and PEP (section 5), jets and QCD studies at LEP (section 6), jets as tools, with applications in Deep Inelastic Scattering Processes, $\gamma \gamma$ collisions, and hard hadronic collisions at the Tevatron and the LHC (section 7). A brief summary (section 8) will conclude this review.

\section{The fragmentation of quarks and gluons}

Quarks and gluons move, due to asymptotic freedom of QCD, as quasi-free particles at short distances of the order of $10^{-15} \mathrm{~cm}\left(10^{-2} \mathrm{fm}\right)$ in the femto-universe. When these coloured objects separate to more than of the order of $1 \mathrm{fm}$, confinement forces become effective which bind the quarks and gluons in hadrons. The hadronisation proceeds through the formation of jets in high energy processes which is driven by two dynamical mechanisms. These mechanisms can be explicated most easily in $e^{+} e^{-}$ annihilation to hadrons, $e^{+} e^{-} \rightarrow q \bar{q}, q \bar{q} g, \ldots, c f$. Fig.2. (i) Quarks which are suddenly accelerated in the production process at short distance and time of the order of $1 / E \ll$ $1 \mathrm{fm}$, will radiate gluons preferentially into a cone of small aperture, $d N / d \Theta^{2} \sim$ $1 / \Theta^{2}$. Subsequently the gluons may split into two gluons or quark-antiquark pairs, and, repeatedly, quarks and gluons again into quark and gluon pairs, so that the original quark fragments finally into a quark/gluon cascade within a narrow cone. (ii) When the coloured quarks on the way out of the femto-universe to large distances separate to more than $1 \mathrm{fm}$, a gluonic flux tube of narrow transverse dimensions builds up which fragments into ordinary hadrons. Similar mechanisms lead to the hadronisation of gluons. In total, the perturbative quark/gluon cascade as well as the partons fragmenting non-perturbatively into hadrons generate jets of particles 

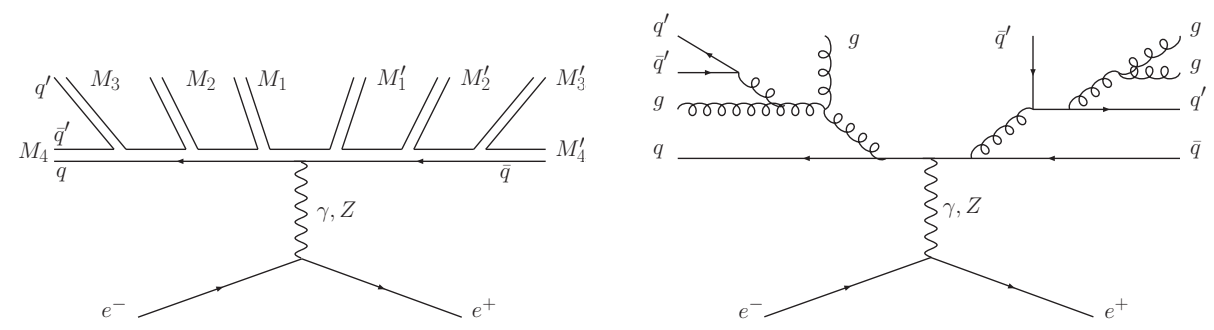

Fig. 2. (a) Quark fragmentation to hadrons induced by confinement forces; (b) Quark/gluon cascades at high energies in QCD.

preserving, in momentum and energy, the original kinematic characteristics of their parent partons.

\subsection{Quark fragmentation}

When quarks and antiquarks in high energetic processes, like $e^{+} e^{-} \rightarrow q \bar{q}$, separate from each other, a linear gluonic flux tube is expected to build up, with energy density of about $1 \mathrm{GeV} / \mathrm{fm}$ and small transverse size, which will confine the two coloured objects. For sufficiently large separations $R \sim 1 \mathrm{fm}$, enough energy will be accumulated in the flux tube so that new quark-antiquark pairs can be created spontaneously and the flux tube breaks up. This expectation is borne out by lattice analyses [56] which, in static approximation, support this picture qualitatively. Beyond the short-distance regime, the potential between heavy quarks rises linearly with distance, $V(R)=\sigma R$ with $\sigma \approx 1 \mathrm{GeV} / \mathrm{fm}$. However, when the distance between the heavy quarks exceeds a value of about $1.2 \mathrm{fm}$, light quark pairs $q \bar{q}$ are created and the heavy-quark $[Q \bar{Q}]$ system breaks up into two separate mesons $[Q \bar{q}]$ and $[\bar{Q} q]$.

Repeating this break-up process, adjacent quarks and antiquarks coalesce to hadrons with small transverse momenta of the order of $350 \mathrm{MeV}$ so that narrow jets of collimated hadrons are generated [37/38. If the partition of energies in $q \rightarrow h_{\left[q \bar{q}^{\prime}\right]}+q^{\prime}$ scales with the energy of the primary quark, the number density of hadrons $D(z)$ observed with energy $z=E^{h} / E_{q}$ obeys, for a single species, the recursion formula [37]

$$
D(z)=f(1-z)+\int_{z}^{1} f(\eta) F(z / \eta) \frac{d \eta}{\eta},
$$

with $f(\zeta)$ denoting the break-up probability for fractional energy $\zeta$. This equation states that the primary meson might be the first in rank primary meson, with probability $f(1-z) d z$, or if not, then the first-rank primary meson has left a momentum fraction $\eta$ with probability $f(\eta) d \eta$, and in this remaining cascade the probability to find $z$ in $d z$ is $F(z / \eta) d z / \eta$. Dividing out by $d z$ gives the above equation. The probability $f(\zeta)$, which must be determined experimentally, is generally parametrised as a polynomial $\sim(1-\zeta)^{\beta}$ or as an exponential $\sim \zeta^{-1}(1-\zeta)^{\beta} \exp [-\alpha / \zeta]$ in string fragmentation.

From this picture two important consequences can be derived.

(i) Solving the evolution equation generates a pole in $z \rightarrow 0$, most easily seen for polynomial probabilities,

$$
D(z) \rightarrow \frac{\text { const }}{z} \text { for } z \rightarrow 0
$$

Thus the fragmentation mechanism predicts a large number of soft (low energy) hadrons in the jets, i.e. a long constant plateau in the rapidity $y=\log z^{-1}$. 
(ii) Summing up the hadron charges in the jets reflects the charge of the parent quark. If $u, d, s$ quark pairs were created in the flux tube spontaneously with equal probabilities, the sum would measure the charge exactly. However, since $s$-quarks weigh a little more than $u$ - and $d$-quarks, the probabilities for spontaneous quarkpair creation deviate from $1 / 3$ by a small amount and a small fraction of the charge leaks into the plateau:

$$
\left\langle Q_{q}\right\rangle=\sum_{h \in j e t} e^{h}=e_{q}-\gamma .
$$

In the parton model language 37 one finds $\gamma \approx 0.067$, i.e. $\left\langle Q_{u}\right\rangle=0.60,\left\langle Q_{d}\right\rangle=$ $\left\langle Q_{s}\right\rangle=-0.40$. The close relation to the ideal values $+2 / 3$ and $-1 / 3$ therefore allows the efficient tagging of the parent quark charges in the jets. In practise, things are more involved in perturbative QCD. Jet-charge studies have been undertaken extensively at LEP [57, where a lot of data are available at the $Z$ peak and flavour-tagged results 58 , distinguishing between the light-quark, charm and bottom contributions, have been obtained.

The light quark fragmentation to mesons can effectively be described by the fragmentation function

$$
D(z)=(1+\beta) \frac{1}{z}(1-z)^{\beta} \text { with } \beta \sim 0.2,
$$

for small jet energies $\sim 7 \mathrm{GeV}$. For higher energies QCD predicts a stronger fall-off of the spectrum. [For a detailed overview of quark fragmentation to various types of mesons and baryons see [5960.]

The fragmentation function of the heavy $c, b$-quarks behaves rather differently. It was recognized very early 6162 that a heavy flavoured meson (containing a charm or bottom quark and a light antiquark) retains a good fraction of the momentum of the primordial heavy quark. Thus, due to the inertia of the heavy quarks, the fragmentation function of a heavy quark should be much harder than that of a light hadron. Estimating the size of the transition amplitude by the energy transfer in the break-up process $Q \rightarrow h_{[Q \bar{q}]}+q$, the fragmentation function behaves, for example, as 63

$$
D_{Q}(z) \sim \frac{1}{z\left[1-\frac{1}{z}-\frac{\epsilon_{Q}}{1-z}\right]^{2}} \text { with } \epsilon_{Q} \sim \Lambda^{2} / M_{Q}^{2},
$$

with $\Lambda \sim 200 \mathrm{MeV}$ denoting the strong interaction scale. The spectrum develops a narrow maximum near $z_{0} \sim 1-\sqrt{\epsilon_{Q}}$. This form describes the essential characteristics of the hard spectra of $Q$-flavoured mesons in the heavy $c, b$ jets with $M_{c} \sim 1.5$ $\mathrm{GeV}$ and $M_{b} \sim 4.5 \mathrm{GeV}$. For more recent works on the heavy quark fragmentation, see 64,65,66,67,68,69].

It should be pointed out that at higher energies, where the heavy quarks are produced with momenta much larger than the heavy quark mass, one expects important perturbative QCD corrections, enhanced by the powers of the logarithms of the transverse momenta to the heavy quark mass, which modify the shape of the fragmentation functions. These effects can be implemented using the framework of an evolution equation which is discussed later in this review. They have to be incorporated in the analysis of data.

After the discovery of quark jets in 1975 in $e^{+} e^{-} \rightarrow q \bar{q}$ at SLAC, detailed studies in understanding the hadronisation process, and hence the energy-momentum profiles of the quark jets, were initiated in 1977 by Feynman and Field [37. In their approach, the initial quarks and antiquarks produced in $e^{+} e^{-}$annihilation fragmented independently in a cascade process, $q \rightarrow q+\left(\bar{q}^{\prime} q^{\prime}\right) \rightarrow h_{\left(q \bar{q}^{\prime}\right)}+q^{\prime}$, conserving the charge and 
other flavour quantum numbers at each step of this cascade. To determine the energymomentum profile, light-cone variables $p=\left(p_{+}, p_{-}, \mathbf{p}_{T}\right)$ were used with $p_{-} \ll p_{+}$, where $p_{ \pm}=E \pm p_{\|}$. The fragmentation $q \rightarrow h+q^{\prime}$ is then affected through a primordial fragmentation function

$$
f_{q}^{h}(z)=1-a+3 a(1-z)^{2}, \quad z=\frac{\left(E+p_{\|}\right)_{\mathrm{h}}}{\left(E+p_{\|}\right)_{\mathrm{q}}},
$$

with $a$ an adjustable energy-independent parameter, fixed by data. As already discussed, this gives rise to an scale-invariant longitudinal energy distribution of hadrons in a jet. Heavy quark fragmentation (for example of a charm quark into a $D$ meson) is encoded by a different primordial $c \rightarrow D$ fragmentation function, as already discussed in this section above. The $\mathbf{p}_{T}$-distribution $\left(\mathbf{p}_{T}\right.$ is the transverse momentum measured with respect to the jet-axis, which can be identified with the direction of the fragmenting quark, for the time being) was implemented in terms of a Gaussian function, characterised by $\sigma_{q} \simeq 0.35 \mathrm{GeV}$, also determined phenomenologically: $g\left(p_{T}^{2}\right)=\left(2 \sigma_{q}^{2}\right)^{-1} \mathrm{e}^{-p_{T}^{2} / 2 \sigma_{q}^{2}}$. Like the flavour quantum numbers, $\mathbf{p}_{T}$ is locally compensated, implying that an $r^{\text {th }}$-rank primary meson has a momentum $\mathbf{k}_{T}(r)$, with $\mathbf{k}_{T}(r)=\mathbf{q}_{T r}-\mathbf{q}_{T(r-1)}$. The striking feature of the Feynman-Field jet is its simple algorithm with the phenomenological profile determined in terms of a few parameters, which provided an adequate description of the non-perturbative aspects of jets initiated by quarks.

\subsection{Gluon fragmentation}

The fragmentation of gluon jets follows rules similar to quarks. Two paths had been chosen in the analysis of PETRA jets. The properties of $g$-jets may be described either as a nearly flavour-neutral average [31] of $u, d$ and, with reduced probability, $s$-quark jets, or, alternatively, gluon jets may be reinterpreted as a superposition of quark and antiquark jet pairs [32] with the spectra derived from the $g \rightarrow q \bar{q}$ splitting function. In any case, the transition from gluons to quarks $g \rightarrow q \bar{q}$ in creating the leading particle will soften the gluon fragmentation compared with the quark fragmentation, accounted for effectively by raising the power fall-off towards $z \rightarrow 1$ of the fragmentation function of the order of $\sim 1.5$.

\subsection{Hadronisation Models}

Quark and gluon configurations created at small distances must transform to bundles of hadrons due to confinement at large distances. The transformation requires nonperturbative mechanisms and, therefore, cannot be treated rigorously at the present time. Instead, models have been developed which describe this step at various levels of sophistication.

\subsubsection{Independent jet fragmentation}

Gluonic flux tubes, built up when coloured objects separate, may hadronize into a jet of collimated hadrons as argued earlier. While the basic picture had first been described for quark jets [37, gluon jets can be analysed similarly when the gluons are either treated globally as partons 31] or split into quark-antiquark pairs, fragmented incoherently again [32. Implementing energy-momentum conservation in the 
overall event was an unsatisfactory element of the model. Nevertheless, independent jet fragmentation has a simple and transparent structure including a small number of parameters. The picture could account quite successfully for the essential properties of two- and three-jet events in $e^{+} e^{-}$annihilation at PETRA and PEP. Thus, it had initially been the right theoretical tool for proving experimentally the gluonic nature of the third jet in three-jet events.

\subsubsection{String model}

Apart from the different choice of the primordial splitting function $f(\zeta)$, motivated by covariance and side-independence of the beginning of the break-up sequence, quark jets in the string model 38 are not very different from independent fragmentation schemes. However, gluons are incorporated quite differently. They generate kinks which locally transfer energy and momentum to the strings stretched between quarks and antiquarks. A small number of hadrons is boosted from the segment between quark and antiquark jets to the segments between quark or antiquark and gluon jets. This string effect has been observed experimentally as reshuffling of hadrons between jets, discussed later.

\subsubsection{Cluster hadronisation}

Quite a different hadronisation mechanism is based on colour pre-confinement [70. Neighbouring coloured partons in cascades arrange themselves in colour-neutral islands with preferentially small invariant masses. In practise, the quark/gluon partons in cascades are evolved down to low invariant masses of the order of several $\Lambda_{\mathrm{QCD}}=O(200 \mathrm{MeV})$, where $\Lambda_{\mathrm{QCD}}$ is the scale parameter specific to QCD and appears in the argument of $\alpha_{s}\left(Q^{2}\right)$. Splitting the gluons in the final step into $q \bar{q}$ pairs, neighbouring quarks and antiquarks form the colourless clusters which may finally decay to standard hadrons according to phase space rules [39]. The reduced number of radiated gluons off heavy quarks and the small number of large invariant masses in the tail of the distribution of the colour-neutral clusters can be covered by nonperturbative string-type compliments to the cluster hadronisation scheme.

Based on these schemes QCD event generators have been constructed which describe hadron spectra at a high level of accuracy. While the prototypes had originally been developed for hadron production in $e^{+} e^{-}$annihilation, the event generators have been expanded to proton-(anti)proton collisions and complimented by programs for lepton-nucleon collisions. The modern versions of PYTHIA 4950, HERWIG [51], SHERPA [52] and others involve the cascading of quarks/gluons in the final and the $p / \bar{p}$ initial states, and string or cluster hadronisation in the final states. For multijet final states, frequently produced at high energies in colliders, elaborate techniques have been developed, based on the relation $71 \operatorname{PS}\left(Q^{2}\right)=$ $\operatorname{ME}\left(Q^{2}\right) \times$ Sudakov factor $\left[Q_{\max }^{2} \rightarrow Q^{2}\right]$, to accomplish smooth transitions between quark/gluon parton showers (PS) and well separated multijet final states described by fixed-order perturbation theory matrix elements (ME) squared.

\subsection{Inclusive jet measures}

In this section we discuss some inclusive jet measures which have played an important role in the quantitative tests of QCD. The first of these is the observable called 
sphericity which played a central role in the discovery of quarks jets at SPEAR. In its tensorial form it is defined as follows [23]:

$$
S_{\alpha \beta}=\frac{\sum_{i} p_{i \alpha} p_{i \beta}}{\sum_{i} \mathbf{p}_{\mathbf{i}}^{2}}
$$

which can be diagonalised obtaining the principal axes $\mathbf{n}_{\mathbf{1}}, \mathbf{n}_{\mathbf{2}}$ and $\mathbf{n}_{\mathbf{3}}$ with corresponding eigenvalues $Q_{1}, Q_{2}$ and $Q_{3}$. The $Q_{i}$ can be ordered $Q_{1}<Q_{2}<Q_{3}$ and normalised so that $Q_{1}+Q_{2}+Q_{3}=1$. Then the squares of the transverse momenta are minimal with respect to the axis $\mathbf{n}_{\mathbf{3}}$ and the sphericity $S$ is given by

$$
S=\frac{3}{2}\left(1-Q_{3}\right)=\frac{3}{2}\left(Q_{1}+Q_{2}\right)
$$

with the sphericity axis equal to $\mathbf{n}_{\mathbf{3}}$. For events with two particles with equal and opposite momenta (ideal two-jet event) we have $S=0$ and $S \rightarrow 1$ for completely isotropic events. Because of the normalisation $Q_{1}+Q_{2}+Q_{3}=1$ only two of the eigenvalues are needed to characterise an event. For example one can take in addition to $\mathrm{S}$ the so-called aplanarity $A p$, which is

$$
A p=\frac{3}{2} Q_{1}=\frac{3}{2} \min \frac{\sum_{i}\left|\mathbf{p}_{\mathbf{i} \mathbf{T}, \mathbf{o u t}}\right|^{2}}{\sum_{i} \mathbf{p}_{\mathbf{i}}{ }^{2}} .
$$

The aplanarity $A p$ minimises the transverse momenta with respect to a plane. Events with small $A p$ are almost planar. The jet variables of an event, $Q_{1}, Q_{2}$ and $Q_{3}$ can be plotted inside a triangle as shown in Fig. 3. in which events obtained by the TASSO Collaboration at PETRA are shown. In this plot planar events are found in the strip with small $A p, 2$-jet events have in addition also small $S$. Alternatively, sphericity can be defined as 23 .

$$
S=\frac{3}{2} \min \frac{\sum_{i}\left|\mathbf{p}_{\mathbf{i} \mathbf{T}}\right|^{2}}{\sum_{i}\left|\mathbf{p}_{\mathbf{i}}\right|^{2}} .
$$

Here, $\mathbf{p}_{\mathbf{i}} \mathbf{T}$ are the transverse momenta of all produced hadrons in the final state event relative to an axis chosen such that the numerator is minimised.

The method based on the sphericity tensor, first applied to the analysis of the 3 -gluon decay of the $\Upsilon$ resonance [72] and to the analysis of $q \bar{q} g$ events [40, has the advantage that the eigenvalues $Q_{i}$ and the principal axes $\mathbf{n}_{\mathbf{i}}$ and from this $S$ and $A p$ can be calculated quite easily. Since in these jet measures the momenta enter quadratically, the high momentum particles are weighted stronger in the calculation of $S$ and $A p$. Also, these variables are not invariant against clustering of particles and depend strongly on details of the fragmentation of quarks (and gluons) into hadrons. This has, for example, the effect, that the sphericity changes if a particle momentum splits up by decay, as for example, $\rho^{0} \rightarrow \pi^{+} \pi^{-}$or by fragmentation, for instance $q \rightarrow q^{\prime}+$ meson into two or more momenta. Therefore these variables are also sensitive to the emission of soft or collinear gluons.

There exist other variables which are infrared safe (this term is used for observables which are free of divergences when calculated in perturbation theory in the limiting case of low energy radiation) and which depend on linear sums of momenta. Known examples are thrust $T$ and acoplanarity $A$ which are defined by

$$
\begin{gathered}
T=\max \frac{\sum_{i}\left|\mathbf{p}_{\mathbf{i L}}\right|}{\sum_{i}\left|\mathbf{p}_{\mathbf{i}}\right|}, \\
A=4 \min \left(\frac{\sum_{i}\left|\mathbf{p}_{\mathbf{i} \mathbf{T}, \mathbf{o u t}}\right|}{\sum_{i}\left|\mathbf{p}_{\mathbf{i}}\right|}\right)^{2} .
\end{gathered}
$$




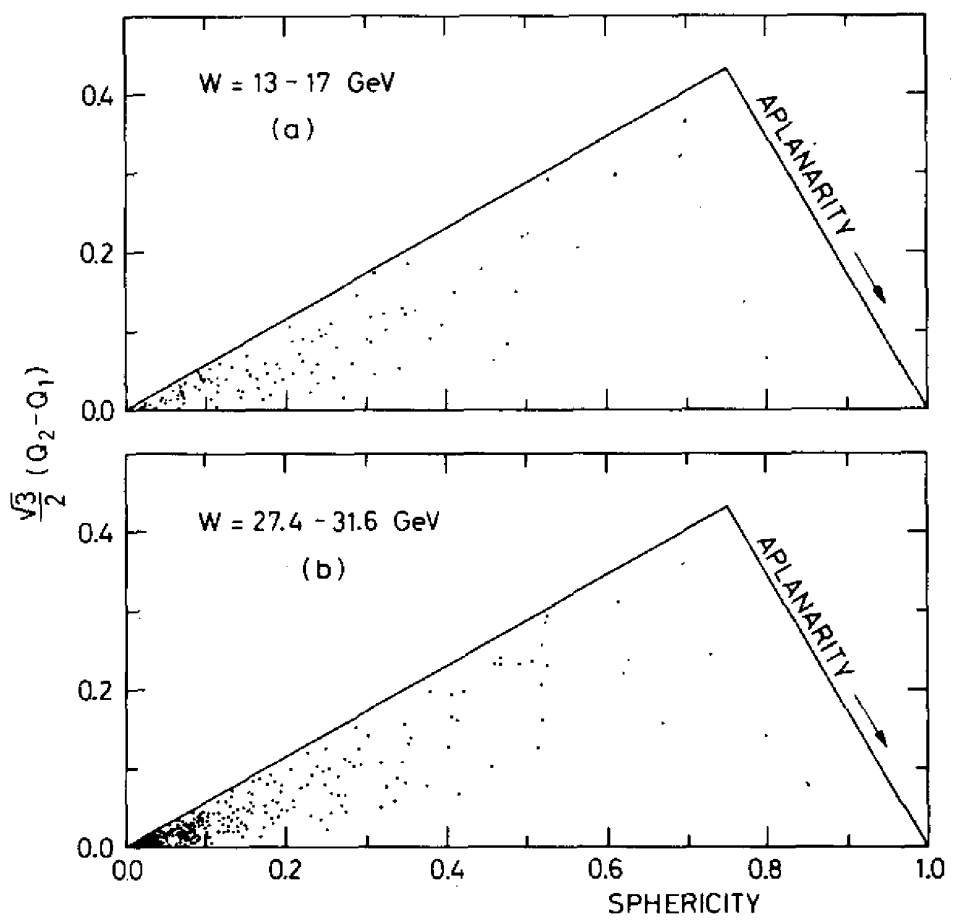

Fig. 3. Distributions of the $e^{+} e^{-} \rightarrow$ hadron events as a function of aplanarity and sphericity defined in the text for the low (a) and high (b) energy PETRA data (TASSO [25]).

For thrust $T$, which was introduced in 7374 , the thrust axis $\mathbf{n}$ is obtained by maximising the longitudinal momenta $\mathbf{p}_{\mathbf{i L}}$ along it. $\mathrm{T}$ varies in the range $0.5<T<1.0$, where the lower limit corresponds to isotropic events and the upper limit to completely collinear configurations. In a similar way for spherocity $S^{\prime}$, defined as 75 ]

$$
S^{\prime}=\left(\frac{4}{\pi}\right)^{2} \min \left(\frac{\sum_{i}\left|\mathbf{p}_{\mathbf{i} \mathbf{T}}\right|}{\sum_{i}\left|\mathbf{p}_{\mathbf{i}}\right|}\right)^{2},
$$

the $\left|\mathbf{p}_{\mathbf{i} \mathbf{T}}\right|$ is minimised with respect to a unit vector $\mathbf{n}$. It lies between 0 and 1 for configurations from collinear to fully isotropic events. Similar to $A p$ the acoplanarity is obtained in such a way that the $\mathbf{p}_{\mathbf{i} \mathbf{T} \text {,out }}$ is minimal with respect to a plane. Planar event must have small $A$ values. For massless particles $A$ varies between 0 and $2 / 3$.

Various other jet measures have been proposed: For example a generalisation of thrust to three clusters instead of two, called triplicity [75] or jettiness [40]. A variable introduced for the analysis to verify the existence of four-jet events is the variable tripodity $D_{3}$ [76]. These and other jet variables will be defined explicitly when they are used to interpret specific final state data in $e^{+} e^{-}$annihilation in later sections.

\subsection{Jet algorithms}

Classifying multi-particle final states qualitatively in jets with high energies is straightforward for a coarse picture. However, when the picture is refined to a high level of precision, algorithms must be employed which define the jets in a rigorous way. In addition, when experimental measurements are compared with theoretical predictions, 
the algorithms used in the experimental analyses must conform with the algorithms adopted in the theoretical analyses.

A multitude of algorithms 77 has been developed to describe jets at high energies. A few representative examples should characterise the two classes, sequential recombination and cone algorithms. Recombination algorithms have been introduced originally in $e^{+} e^{-}$annihilation, while cone algorithms have been used frequently at hadron colliders so far. We shall discuss some of these algorithms later while discussing jets in hadronic collisions.

\subsubsection{Sequential recombination algorithms}

The JADE algorithm 44 is a prominent representative for recombination algorithms applied in $e^{+} e^{-}$annihilation. Particles are clustered in a jet iteratively as long as their distance remains less than a prescribed cut-off value. The distance of two particles is defined by the invariant mass of the pair:

$$
y_{i j}=2 E_{i} E_{j}\left(1-\cos \theta_{i j}\right) / E_{c m}^{2} .
$$

If the criterion $y_{i j} \leq y_{\text {cut }}$ is fulfilled, the particles $i$ and $j$ are combined to a compound by adding up energy and momentum, for instance, and the recombination continues by pairing the compound with the next particle $k$. The procedure stops after all particles are associated with jets. The cut-off value $y_{c u t}$ is generally chosen in the range from $10^{-1}$ down to $10^{-3}$.

The presence of $E_{i} E_{j}$ in the numerator of $y_{i j}$ means that two soft particles moving in opposite directions can get combined into a single particle in the early stages of clustering, which is counter-intuitive to the idea of a jet as consisting of particles restricted in the angular dimension. Apart from this, JADE algorithm leads to a structure in higher orders of pertrurbation theory which does not allow itself to be expressed in a compact resummed form. Technically, this means that the double logarithms of the type $\alpha_{s}^{n} \ln ^{2 n} y_{\text {cut }}(n=1,2, \ldots)$, which arise in higher orders of perturbation theory and which should be resummed to have the correct perturbative form in a limited kinematic region, named after Sudakov, are either not present or not discernible easily. To rectify both of these shortcomings, the JADE algorithm has been improved by substituting $E_{i} E_{j} \rightarrow \min \left[E_{i}^{2}, E_{j}^{2}\right]$ in the DURHAM algorithm [78]. This amounts to defining the distance by the minimal transverse momentum $k_{t}$ of the particles in nearly collinear pairs.

The concept has been transcribed to hadron colliders [7980, where the total subenergy is experimentally not well defined, by switching to un-normalised measures and replacing the angles between particles by the differences of the rapidities $y=$ $1 / 2 \log \left(E+p_{z}\right) /\left(E-p_{z}\right)$ along the beam axis and the azimuthal angles $\phi$ in the plane transverse to the beam axis,

$$
d_{i j}=\min \left[p_{t i}^{2 p}, p_{t j}^{2 p}\right]\left[\left(y_{i}-y_{j}\right)^{2}+\left(\phi_{i}-\phi_{j}\right)^{2}\right] / R^{2},
$$

with $p_{t i(j)}$ denoting the transverse momenta of the particles with regard to the beam axis. The jet parameter $R$ is chosen of the order of one. Since the individual quantities $\left(y_{i}-y_{j}\right),\left(\phi_{i}-\phi_{j}\right), p_{t i}$ and $p_{t j}$ are all invariant under longitudinal boosts, the distance measure $d_{i j}$ is also longitudinally invariant. Recombination with the beam jets is controlled by the observable $d_{i B}=p_{t i}^{2 p}$, included parallel to the measure $d_{i j}$ when recombining all the particles to jets with non-zero transverse momenta and beam jets. Originally, the power parameter $p$ had been chosen 1 in the $k_{t}$ algorithm [78] and 0 in the Cambridge/Aachen algorithm [81]. However, clustering involving hard particles are favoured by choosing $p=-1$ in the anti- $k_{t}$ algorithm 82 . This algorithm, which 
makes jets grow outwards from hard seeds as intuitively expected, is the preferred tool for LHC analyses.

\subsubsection{Cone algorithms}

Cone algorithms had been introduced in QED to tame infrared and collinear singularities encountered in photon radiation off charged particles. The concept has been translated to QCD in formulating the Sterman-Weinberg jets [45. Defining 2-jet events as hadron configurations in which all but a fraction $\epsilon$ of the total energy is contained in cones of half-angle $\delta$ around the central event axis, the ratio

$$
\frac{\sigma_{2}}{\sigma}=1-\frac{32}{3} \frac{\alpha_{s}}{2 \pi} \log \frac{1}{\delta} \log \frac{1}{\epsilon}
$$

describes the 2 -jet fraction of hadronic events in $e^{+} e^{-}$annihilation in the leading logarithmic approximation.

The transition to hadron collisions has been formulated again by adopting the definition of distances based on rapidities and azimuthal angles. The clustering requires a seed location; the 4-momentum of the cluster is determined by summing the 4-momenta of all the objects within a distance $R=\sqrt{\left(y-y_{c}\right)^{2}+\left(\phi-\phi_{c}\right)^{2}}$ from the seed $\left(y_{c}, \phi_{c}\right)$. In one variant, used in the analysis of the Run II Tevatron data, the 4-momenta are summed using the so-called E-scheme 83] (this should not be confused with the E-scheme in the analysis of the jets in $e^{+} e^{-}$annihilation), $\left(E, p_{x}, p_{y}, p_{z}\right)=\sum\left(E, p_{x}, p_{y}, p_{z}\right)_{i}$, and the various variables are defined as

$$
p_{T}=\sqrt{p_{x}^{2}+p_{y}^{2}}, \quad y=\frac{1}{2} \ln \left(\frac{E+p_{z}}{E-p_{z}}\right), \quad \phi=\tan ^{-1}\left(p_{y} / p_{x}\right) .
$$

This differs from the Snowmass clustering algorithm 84, used in the analysis of the Tevatron I data, in which the clustering centroid was defined as the $E_{T}$-weighted averages of the rapidity $y$ and the azimuthal angle $\phi$. The cones are either centred around seed particles (defined as those particles setting the initial direction and one sums the momenta of all the other particles around these seed particles within a specified jet measure), an approach which is not infrared safe, or they are defined by grouping all particles into subsets such that the subsets correspond exactly to pre-defined cones. For further discussion of these and related issues, we refer to the works of Seymour 85 and the comprehensive review of jet measures by Salam [77.

\section{Discovering quark jets}

\subsection{Quark jets at SPEAR}

The notion of jets in $e^{+} e^{-}$annihilation is closely connected with the discovery of Bjorken scaling in deep-inelastic electron-nucleon scattering in 1968 at SLAC. As mentioned in the introduction, inelastic electron scattering on protons and neutrons at large spacelike $\left(q^{2}<0\right)$ momentum transfer and large inelasticity $\nu$ can very well be described in terms of an interaction of the spacelike virtual photon with the pointlike constituents of the nucleon, the partons, identified as the $u$ and $d$ quarks inside the proton and neutron [86]. The analogous process with a timelike $\left(q^{2}>\right.$ 0 ) virtual photon is $e^{+} e^{-}$annihilation into a quark-antiquark pair, $e^{+} e^{-} \rightarrow q \bar{q}$, as shown in Fig. 4, where $q$ stands for the quarks $u, d, s, c, b$. As explained already in the 


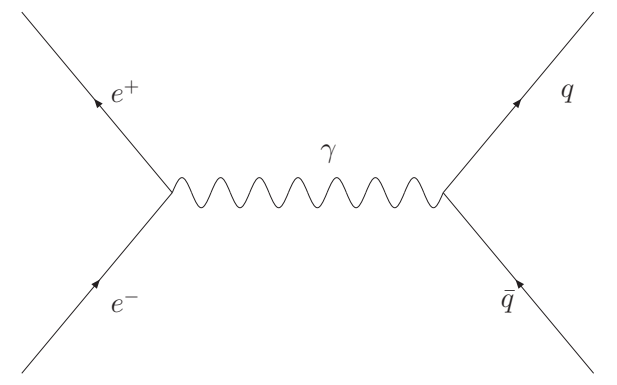

Fig. 4. Born diagram for $e^{+} e^{-} \rightarrow \gamma \rightarrow q \bar{q}$.

introduction, in this simple model the virtual photon from the annihilating electron and positron creates a quasi-free quark and antiquark. The occurrence of real quark and antiquark particles in the final state is prevented by the the fact that they carry non-trivial colour quantum numbers. The quarks and antiquarks transform themselves into hadrons with unit probability under the confinement forces, which act at much later times $t \simeq 1 \mathrm{GeV}^{-1}$. These hadrons should appear in the final state aligned roughly along the momentum direction of the original $q$ and $\bar{q}$, so that two distinct hadron jets with oppositely directed momenta appear in experiments. This discussion mirrors the early "outside-in" picture of jet formation, which was used in the formative stages of jet physics. This approach was later replaced by the so-called "inside-out" description where quark-antiquark pairs were created out of the vacuum before the step of hadronisation. We will discuss the salient feature of this development later.

This simple quark model [18,19:20] was supported by the fact that the total annihilation cross section for hadron production $\sigma\left(e^{+} e^{-} \rightarrow\right.$ hadrons $)$ is given by the square of the quark charges $Q_{f}$ multiplied with the number of colours $N_{C}$ of each quark $q$

$$
\sigma\left(e^{+} e^{-} \rightarrow \text { hadrons }\right) \equiv \sigma_{0}=\frac{4 \pi \alpha^{2}}{3 s} N_{C} \sum_{f} Q_{f}^{2}
$$

where the sum over ' $f$ ' is over all active flavours which can be produced at a given center-of-mass energy $\sqrt{s} ; \alpha$ is the fine structure constant $\alpha \simeq e^{2} / 137$. Dividing by the cross section for the production of a $\mu^{+} \mu^{-}$pair, $\sigma\left(e^{+} e^{-} \rightarrow \mu^{+} \mu^{-}\right)$, one obtains the famous Drell-ratio $R$, defined as

$$
R \equiv \frac{\sigma\left(e^{+} e^{-} \rightarrow \text { hadrons }\right)}{\sigma\left(e^{+} e^{-} \rightarrow \mu^{+} \mu^{-}\right)}=N_{C} \sum_{f} Q_{f}^{2},
$$

which has the numerical value 2 (for $f=u, d, s$ ), and assumes the values $10 / 3$ (for $f=$ $u, d, s, c$ ) and $11 / 3$ (for $, f=u, d, s, c, b$ ), as the threshold for the processes $e^{+} e^{-} \rightarrow$ $c \bar{c}$ and $e^{+} e^{-} \rightarrow b \bar{b}$ are crossed. A recent compilation of the hadronic cross section $\sigma\left(e^{+} e^{-} \rightarrow\right.$ hadrons) and the corresponding ratio $R$ is shown in Fig. 5 (taken from the Particle Data Group [7]) where the various resonances $(\rho, \omega, \phi, J / \psi, \ldots)$ encountered in $e^{+} e^{-}$annihilation and the transition regions are clearly visible. Away from the resonances, the ratio $R$ is almost flat, increasing as a new quark-antiquark threshold is crossed in agreement with the values quoted above. Note that the $t \bar{t}$ threshold (at around $350 \mathrm{GeV}$ ) lies beyond the energies of the $e^{+} e^{-}$collider rings operated so far.

The production of hadron jets in $e^{+} e^{-}$annihilation as a signature of the process $e^{+} e^{-} \rightarrow q \bar{q}$ was suggested by Bjorken and Brodsky already in 1970 [23. However, it was not until 1975 that they were discovered experimentally at SLAC's $e^{+} e^{-}$storage ring SPEAR by the SLAC-LBL Collaboration using the MARK I detector 21] when 


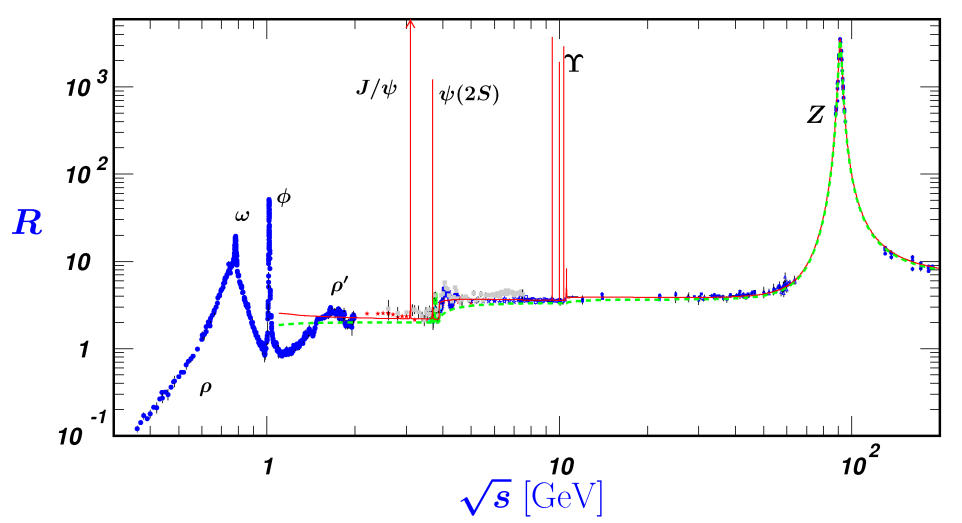

Fig. 5. Measurements of the ratio $R$ as a function of the $e^{+} e^{-}$centre-of-mass energy $\sqrt{s}$ [From PDG 7]].

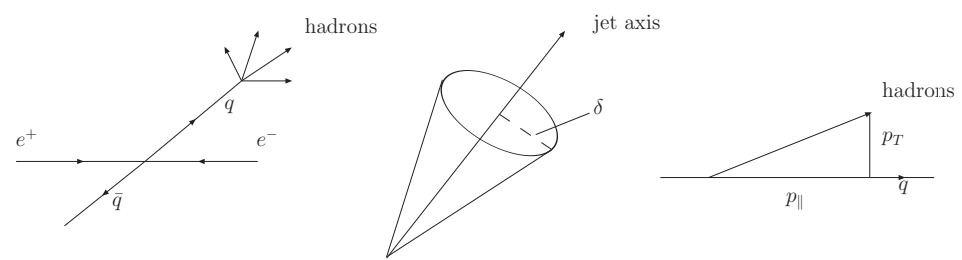

Fig. 6. The process $e^{+} e^{-} \rightarrow q \bar{q} \rightarrow$ jets, with the jets defined using the jet-cone angle $\delta$, arising from limited $p_{T}$ of the hadrons.

high enough centre-of-mass energies $\sqrt{s}$ became available. At low energies, for example at the ADONE ring at Frascati or the original DORIS ring at DESY, it was not possible to see jets because the jet cones were too broad. This is easy to understand if we assume that the transverse momentum $p_{T}$ with respect to the jet direction (which, theoretically is the momentum direction of the original quark $q$ or antiquark $\bar{q}$ ), which are emitted back-to back in the c. m. system, is limited and that the hadron multiplicity $\langle n\rangle$ increases only modestly with $\sqrt{s}$. The jet cone becomes narrower with increasing $\sqrt{s}$, characterised by the mean half angle $\langle\delta\rangle$ of the jet cone (see Fig. 6). At $\sqrt{s}=4 \mathrm{GeV}$ the particle multiplicity is about 6 , so that with $<p_{T}>\simeq$ $0.35 \mathrm{GeV}$ the half-angle of the jet-cone $\left\langle\delta>\simeq<p_{T}><n>/ \sqrt{s} \simeq 0.53 \simeq 30^{\circ}\right.$. This shows that at this energy each of the two jets is broader than $60^{\circ}$ on average.

For establishing the jets it is necessary to determine the jet axis along which the transverse momenta of the produced hadrons are minimised, In the early work of the SLAC-LBL collaboration, the jet axis was defined in terms of the sphericity variable defined earlier. In the SLAC-LBL experiment the mean sphericity was found to be approximately constant as a function of the total $e^{+} e^{-}$-energy $E_{c . m} .=\sqrt{s}$ up to $4 \mathrm{GeV}$ and then it decreases with increasing $E_{c . m .}$. A detailed comparison is shown in Fig. [7, in which the measured sphericity distributions $d \sigma / d S$ at $E_{c . m .}=3.0,6.2$ and $7.4 \mathrm{GeV}$ are compared with the calculated distributions based on a two-jet model and the phase-space. At $3.0 \mathrm{GeV}$ there is no distinction between the two models and the data agree with both. At 6.2 and $7.4 \mathrm{GeV}$ the $S$ distributions are peaked towards low $S$ favouring the jet model. But the $S$ distributions are still very broad. This comparison shows quite clearly that (i) the $E_{c . m}$. must be high enough to see the production of jets in $e^{+} e^{-}$annihilation, and (ii) that even at the higher $E_{c . m}$. energy range of the SPEAR storage ring, the jet structure is visible only through 


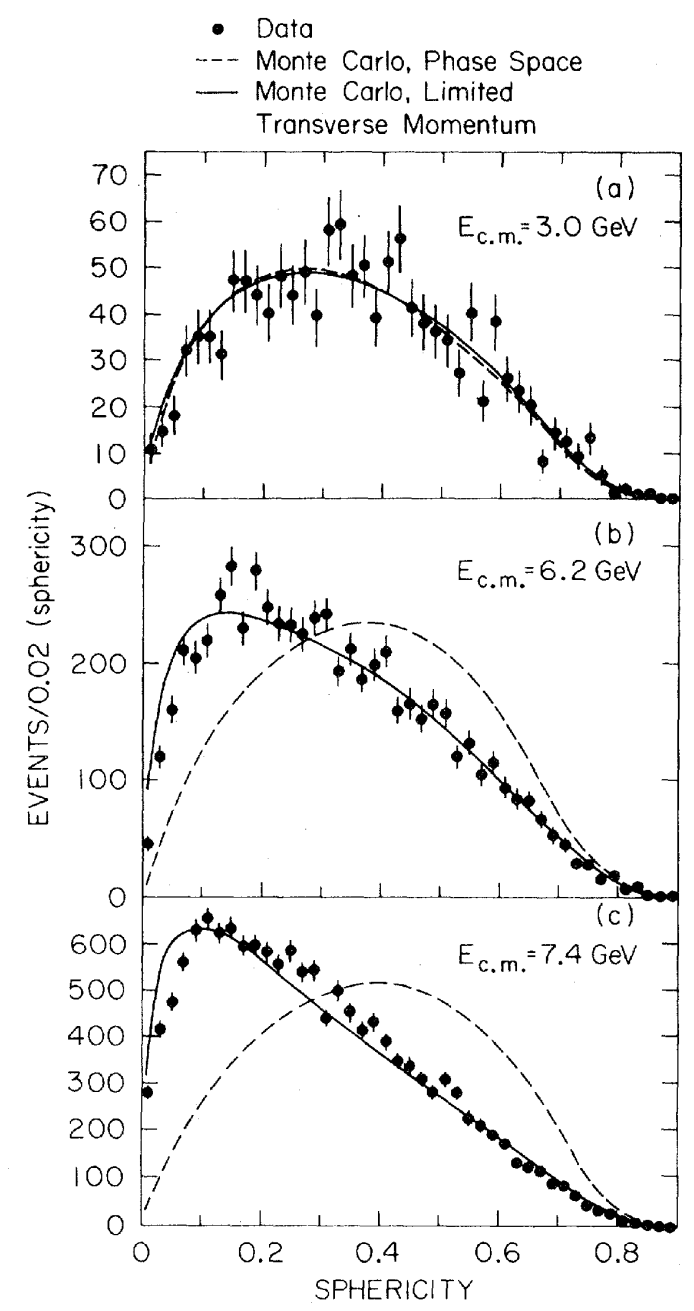

Fig. 7. Observed sphericity distributions for data from MARK I detector, jet model (solid curves), and phase-space model (dashed curves) for (a) $E_{c . m} .=3.0 \mathrm{GeV}$, (b) $E_{c . m .}=6.2$ $\mathrm{GeV}$, and (c) $E_{c . m .}=7.4 \mathrm{GeV}$. (From Ref. [21].)

a detailed comparison with the prediction of an appropriate jet model. Observing the jet structure was easier at PETRA energies, where most of the events have a two-jet topology, which, because of the higher energy had much narrower angular jet-cones. An example of such an event measured by the TASSO collaboration at $E_{c . m .}=31.6 \mathrm{GeV}$, is shown in Fig. 11 (left-hand frame).

Further tests of the underlying quark structure of the jets in $e^{+} e^{-}$annihilation were undertaken at SPEAR. One such test is the measurement of the angular distribution $d \sigma / d \cos \theta$ of the jet axis with respect to the beam direction. This distribution for the production of massless spin $1 / 2$ particles is 87 ]

$$
\frac{d \sigma}{d \cos \theta} \sim 1+\cos ^{2} \theta
$$

The first data came from the SLAC-LBL Collaboration at SPEAR. They did the measurement with transversely polarised $e^{+}$and $e^{-}$beams available at the lower 


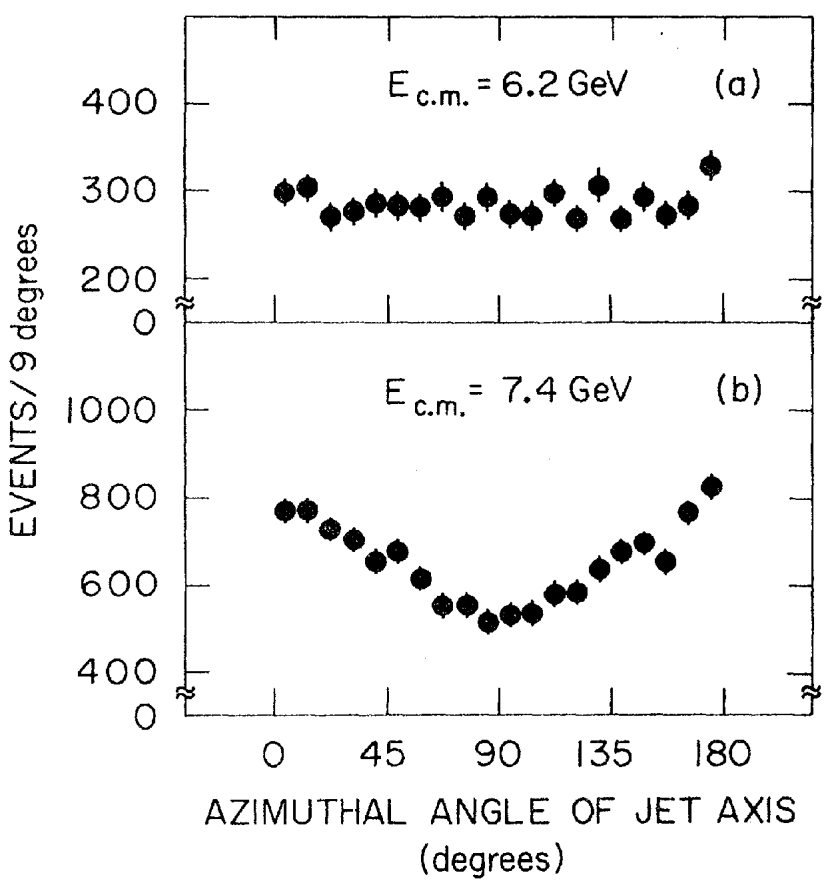

Fig. 8. Observed distributions of jet-axis azimuthal angles from the plane of the storage rings for jet axis with $|\cos \theta| \leq 0.6$ for (a) $E_{c . m} .=6.2 \mathrm{GeV}$ and (b) $E_{c . m}$. $=7.4 \mathrm{GeV}$. (From Ref. 21.)

c.m. energies of the SPEAR ring. With transversely polarised beams the angular distribution has the following form

$$
\frac{d \sigma}{d \Omega} \sim 1+\alpha \cos ^{2} \theta+\alpha P_{+} P_{-} \sin ^{2} \theta \cos 2 \phi,
$$

where $\phi$ is the azimuthal angle of the jet axis with respect to the storage ring plane and $P_{+}$and $P_{-}$are the polarisations of the $e^{+}$and $e^{-}$beams, respectively. The measured $\phi$ distributions (averaged over $\theta$ ) for 6.2 and $7.4 \mathrm{GeV}$ are seen in Fig. 8. At $6.2 \mathrm{GeV}$ the beam polarisations are $P_{+}=P_{-}=0$ and therefore the $\phi$ distribution is isotropic. At $7.4 \mathrm{GeV}$, where $P_{+} P_{-}=0.5$ the characteristic $\cos 2 \phi$ behaviour is observed. From this measurement at SPEAR, the value $\alpha=0.97 \pm 0.14$ [21]22] is in agreement with the expectation for spin $1 / 2$ quarks, $\alpha=1$.

Similar, but less accurate, results were obtained by the PLUTO Collaboration at DORIS for $E_{c . m .}=7.7$ and $9.4 \mathrm{GeV}$ 88. Measurements of the angular distribution of jets at higher energies were also performed at the $e^{+} e^{-}$storage rings PEP and PETRA. Although the beam energies were much higher, yielding a much better defined jet axis, the result $\alpha=1.04 \pm 0.16$ [89] does not have a better accuracy than the SPEAR measurement, which had the benefit of polarised beams. This test of the spin $1 / 2$ nature of the quarks produced in $e^{+} e^{-}$annihilation is very much the same as the verification of the Callan-Gross relation 90 in deep inelastic lepton-nucleon scattering: $F_{2}(x)=2 x F_{1}(x)$, which is also very well satisfied experimentally. 


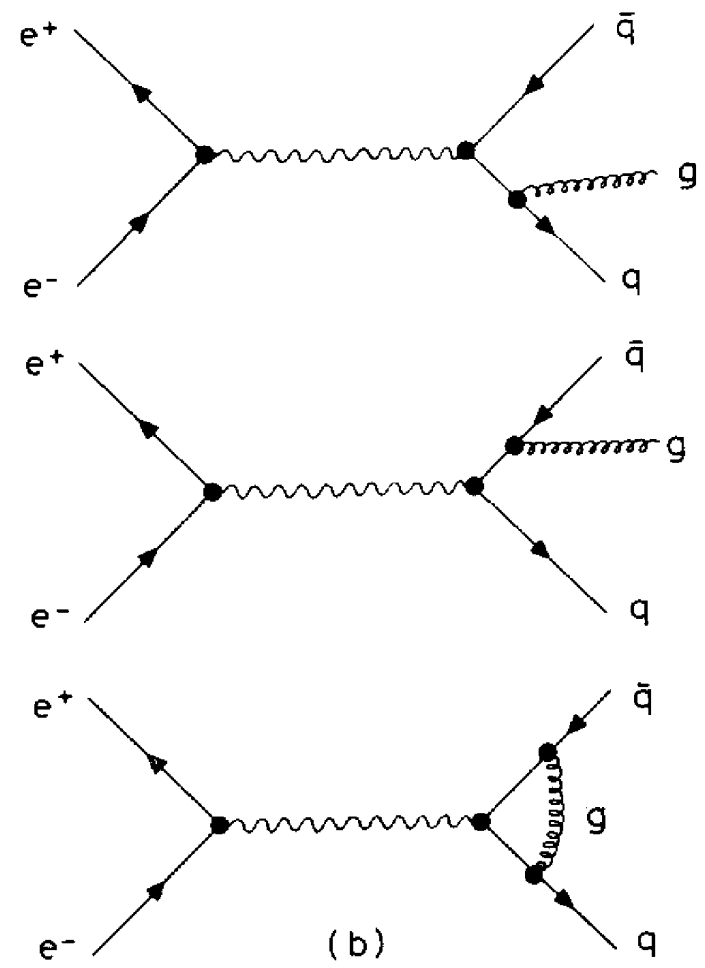

Fig. 9. Lowest order Feynman diagrams contributing to $e^{+} e^{-} \rightarrow q \bar{q} g$ (upper two diagrams) and vertex corrections in $e^{+} e^{-} \rightarrow q \bar{q}(\mathrm{~b})$.

\subsection{Sterman-Weinberg Jets}

The existence proof of jets in QCD was provided by Sterman and Weinberg 45. As already noted, they calculated the cross section $\sigma_{2-\text { jet }}(\epsilon, \delta)$ for the process $e^{+} e^{-} \rightarrow$ 2 - jets, where the jets are defined by two cones in opposite hemispheres with halfangular size $\delta$, having all but a fraction $\epsilon$ of the total c.m. energy. In the general field theory context, jets were anticipated due to the Lee-Nauenberg theorem [91, which states that the transition probability in a theory involving massless particles is finite provided summation over degenerate states is performed.

The Feynman diagrams which contribute in order $\alpha_{s}\left(Q^{2}\right)$ are shown in Fig. 9, For small $\epsilon$ and $\delta$, and to leading order in $\alpha_{s}\left(Q^{2}\right)$ one has

$$
\sigma_{2-\mathrm{jet}}(\epsilon, \delta)=\sigma_{0}\left[1+C_{F} \frac{\alpha_{s}\left(Q^{2}\right)}{\pi}\left(-4 \ln 2 \epsilon \ln \delta-3 \ln \delta-\frac{\pi^{2}}{3}+\frac{5}{2}+O(\epsilon)+O(\delta)\right)\right],
$$

where $\sigma_{0}$ is the lowest order cross section given in Eq. (21), $C_{F}=4 / 3$ and $\alpha_{s}\left(Q^{2}\right)$ is the QCD coupling constant defined in the lowest order

$$
\alpha_{s}\left(Q^{2}\right)=\frac{12 \pi}{\left(33-2 n_{f}\right) \ln \frac{Q^{2}}{\Lambda^{2}}},
$$

where $n_{f}$ is the number of quark flavours. The terms $O(\epsilon), O(\delta)$ neglected by Sterman and Weinberg are all finite, essentially proportional to the phase space and have 
been subsequently worked out 92 . Here $\Lambda$ is a scale parameter, to be determined experimentally, typically of $O(200) \mathrm{MeV}$. As $Q^{2} \rightarrow \Lambda^{2}, \alpha_{s}\left(Q^{2}\right) \rightarrow \infty$, signaling the breakdown of perturbation theory. The above expression for $\alpha_{s}\left(Q^{2}\right)$ also states that $\alpha_{s}\left(Q^{2}\right) \rightarrow 0$ as $Q^{2} \rightarrow \infty$, implying that QCD is an asymptotically free field theory. In the range of $Q^{2}$ where $\alpha_{s}\left(Q^{2}\right) / \pi \ll 1$, one has a controlled perturbative region.

Since, in leading order in $\alpha_{s}\left(Q^{2}\right)$, the inclusive hadronic cross section for $e^{+} e^{-} \rightarrow$ $\gamma \rightarrow$ hadrons is

$$
\sigma\left(e^{+} e^{-} \rightarrow \gamma \rightarrow \text { hadrons }\right)=\sigma_{0}\left(1+\frac{\alpha_{s}\left(Q^{2}\right)}{\pi}\right)
$$

the complement of $\sigma_{2-\text { jet }}(\epsilon, \delta)$ is the 3 -jet cross section

$$
\sigma_{3-\mathrm{jet}}(\epsilon, \delta)=\sigma_{0} \frac{\alpha_{s}\left(Q^{2}\right)}{\pi} C_{F}\left(4 \ln 2 \epsilon \ln \delta+3 \ln \delta+\frac{\pi^{2}}{3}-\frac{7}{4}+O(\epsilon)+O(\delta)\right)
$$

This implies that for typical jet resolutions, a small fraction of hadronic events should consist of three-jets. They were found subsequently in $e^{+} e^{-}$annihilation at PETRA and we shall discuss them later quantitatively.

Another example of a jet measure which can be used with ease to characterise jets is in terms of the invariant mass of the partons $y_{i j}$ emerging from a hard process, defined in Eq. (17). Requiring $y_{i j}>y_{\text {min }}>0$, one avoids both infrared and collinear singularities in a perturbative calculation. The first of such $y_{\min }$-dependent 2 -jet crosssection was calculated in [53], yielding $\left(y_{\min }=y\right)$

$$
\sigma_{2-j e t}=\sigma_{0}\left[1+C_{F} \frac{\alpha_{s}\left(Q^{2}\right)}{2 \pi}\left(-2 \ln ^{2} y-3 \ln y+4 y \ln y-1+\frac{\pi^{2}}{3}+O(y)\right)\right] .
$$

The $O(y)$ terms have been derived in 93 .

The two prescriptions just discussed have been used in the experimental analysis of data concerning jets. Thus, for example, the JADE algorithm [94, used mostly in the analysis of the $e^{+} e^{-}$data at PETRA and PEP, is based on the $y_{\text {min-prescription, }}$ which can be used to classify also muli-jet events. This was subsequently replaced by the $k_{t}$-jet algorithm, as discussed in the preceding section. The modified form of the the cone-prescription is widely used in the analysis of jets in hadroproduction processes.

\section{Gluon jets in Upsilon decays}

The $\Upsilon$ meson first produced in proton-nucleus collisions at FERMILAB 9596 and identified by the $\Upsilon \rightarrow \mu^{+} \mu^{-}$decay was later observed as a narrow resonance with mass $m_{\Upsilon}=9.46 \mathrm{GeV}$ and width $\Gamma_{\Upsilon}=\left(40_{-8}^{+13}\right) \mathrm{keV}$ in the process $e^{+} e^{-} \rightarrow \Upsilon \rightarrow$ hadrons 979899100 101/102. This resonance is a $b \bar{b}$ bound state and has the quantum numbers $J^{P C}=1^{--}$. As the $B \bar{B}$ threshold lies above $m_{\Upsilon}$, the $\Upsilon(9.46)$ state is predicted to decay mainly into 3 gluons $(g)$ in QCD, the massless colour-octet vector particles [103 104 105 106 107/108109] in complete analogy with the decay of orthopositronium into 3 photons [110. While $\Upsilon(9.46) \rightarrow g g g$ is the dominant decay mode, with $3 \%$ probability it can decay also into a photon and 2 gluons, $\Upsilon(9.46) \rightarrow \gamma g g$. Average energies of the three partons were measured as $\left\langle E_{1}\right\rangle \simeq 4.1 \mathrm{GeV}$ for the most energetic of the three gluons, with the other two having energies $\left\langle E_{2}\right\rangle \simeq 3.4 \mathrm{GeV}$ and $\left\langle E_{3}\right\rangle \simeq 2.0$ $\mathrm{GeV}$, respectively 111. in approximate accord with the lowest order QCD matrix elements. However, only the fastest of the three partons yielded a collimated jet of 


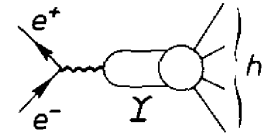

$(a)$

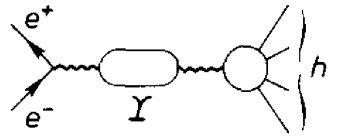

(b)

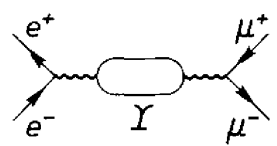

(d)

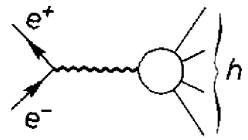

(c)

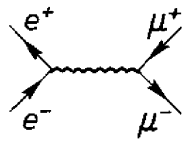

(e)

Fig. 10. Processes contributing to hadronic final states in the $\Upsilon(9.46)$ resonance region: (a) from direct decays of the $\Upsilon$, (b) from the $\Upsilon$ vacuum polarization and (c) from the nonresonant continuum. The $\mu^{+} \mu^{-}$final state can be produced from the $\Upsilon$ vacuum polarization (d), and from the continuum (e). (from Ref. [111]).

hadrons and its detailed phenomenological profile was studied by PLUTO [111112. Phenomenological models, which included the lowest order QCD matrix elements and the fragmentation of the partons (quarks and gluons), were found to be in conformity with a number of inclusive measurements undertaken by PLUTO.

The contributions to the multi-hadron events from the $\Upsilon$ mass region originates from three sources [11], as shown in Fig. 10. The first row in this figure shows the direct decay of the $\Upsilon$ (a), decay through the vacuum polarisation (one-photon decay) (b), and the non-resonating continuum (c). Denoting the corresponding cross sections as $\sigma_{\text {on }}$ (cross-section in the $\Upsilon(9.46)$ energy range), $\sigma^{\mathrm{vp}}$ (cross-section for the $\Upsilon$ vacuum polarisation), and $\sigma^{\text {off }}$ (cross-section for the non-resonant continuum), the cross-section for the $\Upsilon(9.46)$-production with direct decay is $\sigma^{\text {dir }}=\sigma^{\text {on }}-\sigma^{\text {off }}-\sigma^{\mathrm{vp}}$. Since for $\mu^{+} \mu^{-}$final states, a 'direct' production does not exist, the term $\sigma^{\mathrm{vp}}$ can be obtained by scaling the $\mu$-pair production on and off-resonance to the hadron production level. This yields:

$$
\sigma^{\mathrm{dir}}=\sigma^{\mathrm{on}}-\sigma^{\mathrm{off}}-\sigma^{\mathrm{vp}}=\sigma^{\mathrm{on}}-\sigma^{\mathrm{off}}-\sigma^{\mathrm{off}} \frac{\sigma_{\mu \mu}^{\mathrm{on}}-\sigma_{\mu \mu}^{\mathrm{off}}}{\sigma_{\mu \mu}^{o f f}} .
$$

Using $\left(\sigma_{\mu \mu}^{\text {on }}-\sigma_{\mu \mu}^{\text {off }}\right) / \sigma_{\mu \mu}^{\text {off }}=0.24 \pm 0.22[24$ and the number of events in the two energy regions, the $\Upsilon$ direct decay cross section is obtained. This is evaluated as a function of sphericity $S$. The results are shown in Fig. $11 \mathrm{a}, \mathrm{b}, \mathrm{c}$, separately for the off-resonance data, the data at the $\Upsilon$ resonance and the subtracted distribution for the $\Upsilon$ direct decay. These experimental results are compared to the two-jet model based on the Feynman- Field model, already discussed (dash-dotted line in Fig. 11 a) and the predictions of the phase-space model in Fig. 11 c (dashed line) together with the three-gluon decay model (solid line). The off-resonance data are well described by the two-jet model in agreement with the earlier findings at SPEAR. The distribution for the direct decay is shifted to larger sphericity values and is best reproduced by the three-gluon decay model.

The $\Upsilon$ meson is produced at rest. Therefore, the scaled momenta $\mathbf{x}_{\mathbf{i}}=2 \mathbf{k}_{\mathbf{i}} / m_{\Upsilon}$ obey the relations $\left(x_{i}=\left|\mathbf{x}_{\mathbf{i}}\right|\right): \mathbf{x}_{\mathbf{1}}+\mathbf{x}_{\mathbf{2}}+\mathbf{x}_{\mathbf{3}}=0, x_{1}+x_{2}+x_{3}=2$.

Another possibility to describe the configuration of the final state uses the angles between the gluons. For massless gluons the relation between the $x_{i}$ and the angles $\theta_{i}$ is: $x_{i}=\frac{2 \sin \theta_{i}}{\sum_{i} \sin \theta_{i}}$. 


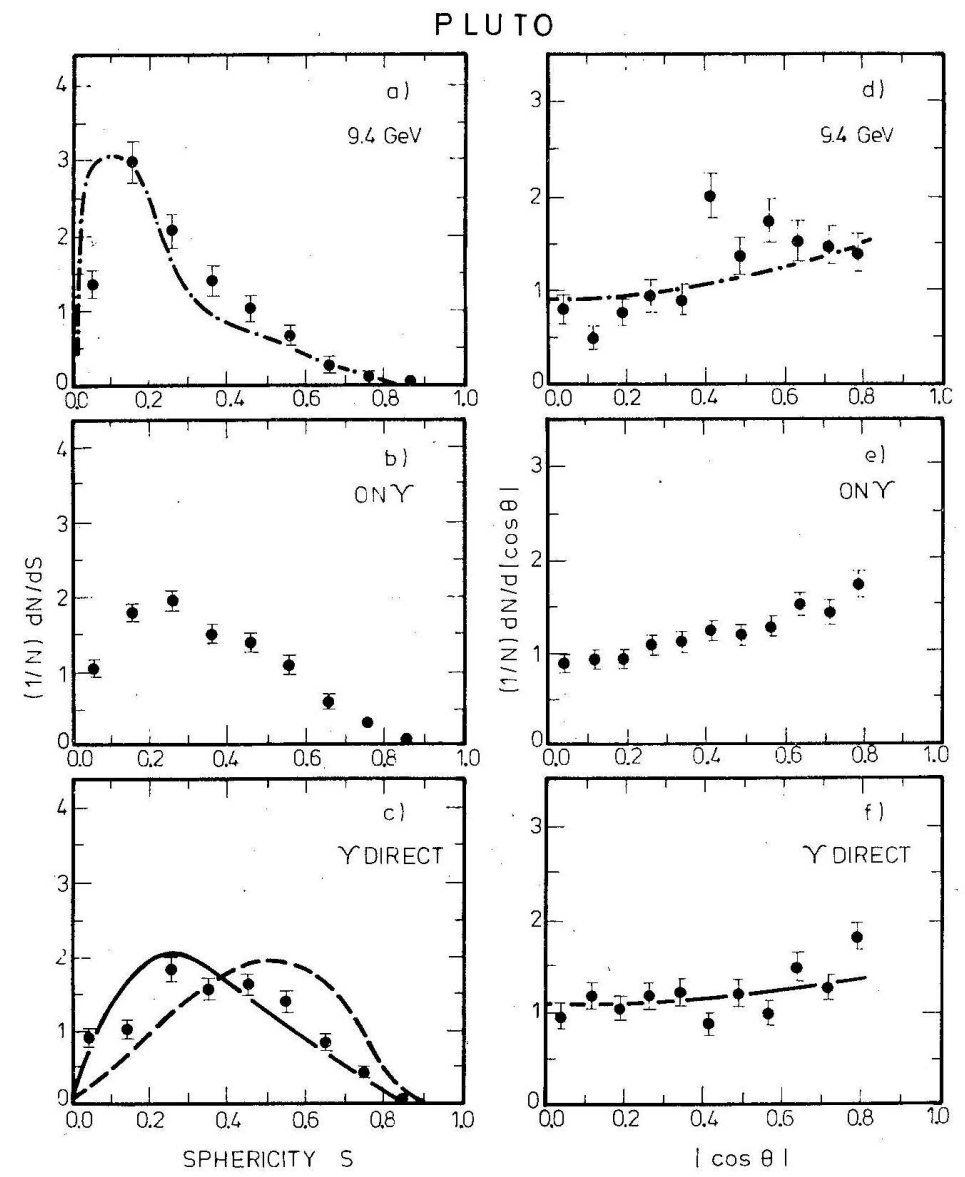

Fig. 11. Differential sphericity distributions and the sphericity angular distributions. The dash-dotted line in (a) represents the two-jet model. The dashed and solid lines in (c) represent respectively phase-space and the three-gluon decay models. The dash-dotted line in (d) is proportional to $1+\cos ^{2} \theta$ and the solid line in (f) to $1+0.39 \cos ^{2} \theta$ (from Ref. 24]).

This relation allows one to characterise the final gluon configuration in the corners of the Dalitz plot $\left(x_{1}=x_{2}=x_{3}=2 / 3\right.$ is the "Mercedes-Star"-like configuration, $x_{1}=x_{2}=1, x_{3}=0$ is a two-jet configuration with the third gluon perpendicular to the direction of the first two and $x_{1}=1, x_{2}=x_{3}=0.5$ is the configuration, where the fastest jet recoils against the two others with $x_{2}=x_{3}$ ). The momentum distribution of the gluon as calculated in leading-order (LO) QCD is [105106 107 108109].

$$
\frac{1}{\sigma} \frac{d^{2} \sigma}{d x_{1} d x_{2}}=\frac{6}{\left(\pi^{2}-9\right) x_{1}^{2} x_{2}^{2} x_{3}^{2}}\left(x_{1}^{2}\left(1-x_{1}\right)^{2}+x_{2}^{2}\left(1-x_{2}\right)^{2}+x_{3}^{2}\left(1-x_{3}\right)^{2}\right) \text {. }
$$

The above cross section formula is the basis for Monte-Carlo model calculations mentioned above. In these models the hard cross section for $\Upsilon \rightarrow 3 g$ must be supplemented with the additional fragmentation of the 3 gluons into hadrons.

To compare the decay $\Upsilon \rightarrow 3 g$ with vector gluons as follows from QCD, also models with scalar gluons have been considered. The momentum distribution corresponding to scalar gluons was derived in 113 leading to the result that they peak at the corners of the Dalitz plot and have zeros in the middle of each boundary. In contrast, vector 


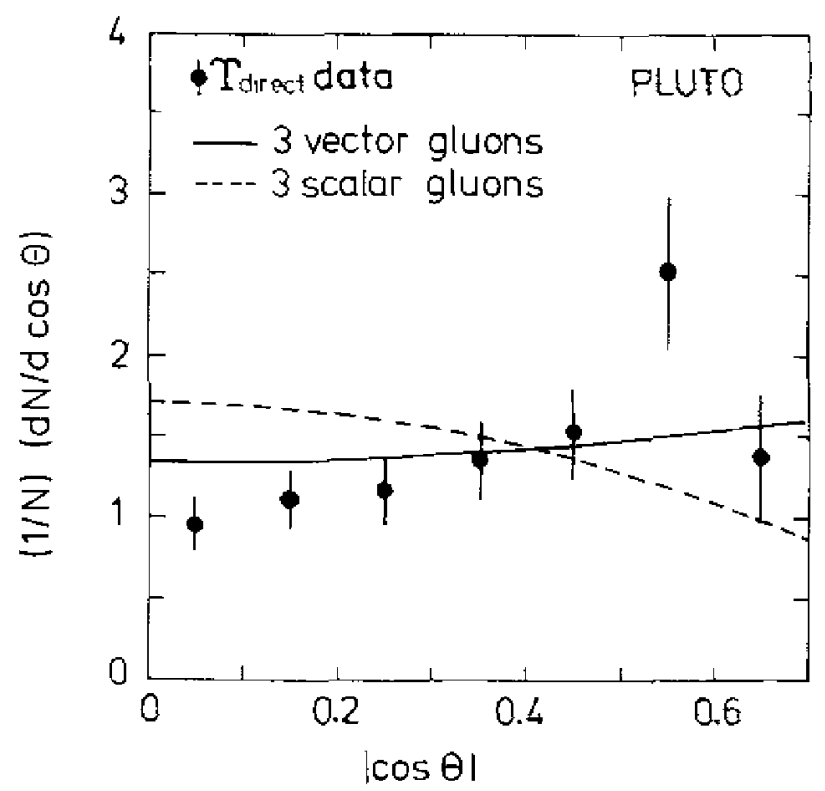

Fig. 12. Corrected experimental distribution in $|\cos \theta|$ from the decays $\Upsilon \rightarrow$ hadrons by the PLUTO collaboration, where $\theta$ is the angle between the thrust axis and the beam axis. The curves are theoretical distributions for $\Upsilon$-decay into vector (solid curve) and scalar (dashed curve) gluons, respectively (from Ref. [11] ).

gluons populate nearly uniformly the Dalitz plot. As the majority of the events have one gluon with very low energy, a 2-jet structure is expected for scalar gluon theories 114 .

The distribution of the gluon direction in space is essentially determined by the QCD theory [105]106]107]108]109]. For vector gluons QCD predicts

$$
W(\cos \theta) \sim 1+0.39 \cos ^{2} \theta,
$$

where $\theta$ is the angle between the most energetic gluon and the momentum of the incoming initial electron (see Fig. 111). Scalar gluons would give rise to the angular distribution 113

$$
W(\cos \theta) \sim 1-\cos ^{2} \theta .
$$

The PLUTO collaboration [111] has measured a number of observables to strengthen the 3 -gluon interpretation of the hadronic $\Upsilon$ decay. The test of vector gluons versus scalar gluon has been done using the angular distribution in $\cos \theta$, where $\theta$ is the angle between the thrust axis and the beam momentum. Theoretical distributions predicted for $\Upsilon$ decay into vector and scalar gluons, respectively, are shown in Fig. 12 compared with the PLUTO measurements [111. The data clearly prefer the vector gluon decay. Similar conclusions have been reached by the LENA collaboration on the basis of their measurements [115].

Further comparisons with the 3-gluon model presented in 111 consist of topological tests with the jet variable thrust, defined earlier, and the variable triplicity $T_{3}$ [75], an extension of thrust to 3 -jet configurations, where the final state particles are grouped into 3 classes with total momentum $\mathbf{P}\left(C_{l}\right), l=1,2,3$. The values of triplicity vary between $T_{3}=1$ for a perfect 3 -jet event and $T_{3}=3 \sqrt{3} / 8$ for completely spherical events. PLUTO data have been analysed also in terms of the fractional energies $x_{i}$ 

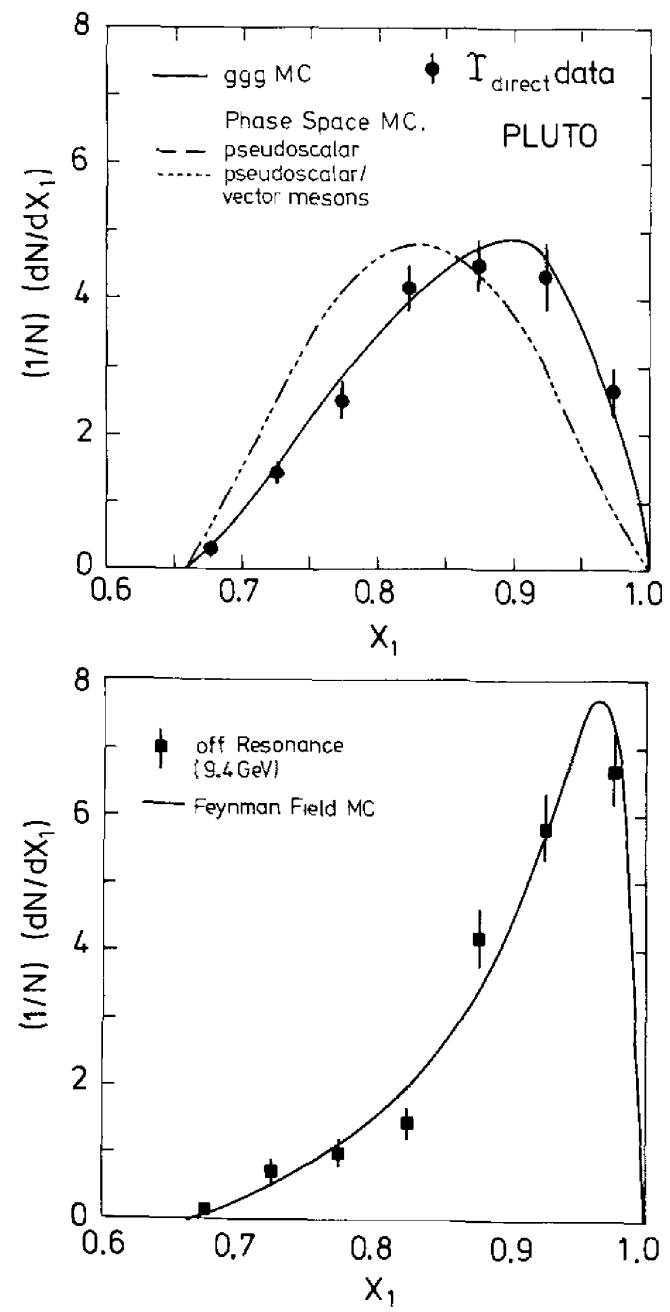

Fig. 13. Experimental distribution of the reconstructed reduced energy $x_{1}$ of the fastest triplicity jet for the $\Upsilon$-direct and off-resonance data taken by the PLUTO collaboration compared to Monte Carlo calculations for various models (from Ref. 111]).

of the jets, where the jet axes are obtained from the triplicity analysis. If the three jets would be completely separated in space, the fractional energies would be independent of the fragmentation of the gluons and would depend, in the lowest order perturbation theory, only on the QCD matrix element $W\left(x_{1}, x_{2}, x_{3}\right)$ given above (see Eq. [31). In Fig. 13] the projections of the two-dimensional histograms, spanned by the axes $x_{3}$ and $\left(x_{1}-x_{2}\right) / \sqrt{3}$, on the $x_{1}$ axis is shown (this is also the distribution of the most energetic triplicity jet). The prediction of the 3 -gluon Monte Carlo model is compared to the data and impressive agreement is obtained, whereas two versions of the phase-space Monte Carlo model fail to do so. (See 112 for a recent reappraisal of the PLUTO experimental analysis). All this information taken together demonstrates that the $\Upsilon$-direct decay data are very well reproduced by the 3 -gluon model while all the other models disagree with $\Upsilon$-direct data. These findings were further backed up later by the CLEO collaboration at the CESR storage ring at Cornell [116] and the ARGUS collaboration at the DORIS ring at DESY [117. 


\section{Jets in QCD and at PETRA and PEP}

To put the contents of this chapter in historical perspective, we would like to introduce the main detectors which played an important role in the development of jets and detailed tests of QCD at PETRA and PEP. In doing this, however, we will be very brief and refer the interested readers to the review by Gidal et al. [118, which is a compendium of the properties and performance characteristics of the major high energy physics detectors in that epoch, and the review by Lynch [119]. In alphabetical orders, these detectors were CELLO, JADE, MARK-J, PLUTO and TASSO (all located at the PETRA $e^{+} e^{-}$rings at DESY, Hamburg), DELCO, the High Resolution Spectrometer HRS, the Magnetic Calorimeter MAC, MARK II, MARK III, and the Time Projection Chamber TPC (all located at the PEP $e^{+} e^{-}$ring at SLAC, Stanford). As already mentioned in the introduction, PETRA started data runs in 1978 with the maximum beam energy of $23.6 \mathrm{GeV}$ and PEP came a little later in 1980 having the maximum beam energy of $15 \mathrm{GeV}$. These detectors were involved in measurements for almost a decade, ending their runs as LEP started taking data at higher energies.

\subsection{Jet-like distributions from the weak decays of heavy quarks}

The process $e^{+} e^{-} \rightarrow q \bar{q} g$ leads to $p_{T^{-}}$-broadening of the quark jets, leading eventually to three-jet topologies as the centre-of-mass energy increases. There is another source of $p_{T}$-broadening in $e^{+} e^{-}$annihilation due to the production of a heavy quark-antiquark pair $e^{+} e^{-} \rightarrow Q \bar{Q}$, and the subsequent weak decays of the heavy quarks/hadrons. For the centre-of-mass energies available at the $e^{+} e^{-}$colliders PEP and PETRA, the heavy quarks whose production and decays had to be correctly taken into account were the charm-anticharm $(c \bar{c})$ and bottom-antibottom $(b \bar{b})$ pairs. Sampling the theoretical predictions of the top quark mass in the PEP and PETRA era, most guesses put it around $10-15 \mathrm{GeV}$ [120121]. Hence, the production of a topantitop pair was widely anticipated at these colliders 122123, and their characteristic jet topologies were worked out in the context of the Cabibbo-Kobayashi-Maskawa (CKM) 6-quark model [124|125]. However, as subsequent developments showed, there were no top quarks to be seen at PETRA and PEP (or at LEP). Thanks to the Fermilab-Tevatron [126, the top quark has a measured mass of about $173 \mathrm{GeV}$.

The event topology in $e^{+} e^{-}$annihilation is sensitive to the onset of $Q \bar{Q}$ threshold. The data in the center-of-mass energy in the range $9.4 \mathrm{GeV} \leq \sqrt{s} \leq 17.0 \mathrm{GeV}$ was analysed [127] in terms of the measures of jettiness, $\langle S\rangle$ and $\langle 1-T\rangle$, which showed a clear step as the $B \bar{B}$ threshold is crossed. The data taken by the PLUTO collaboration 24] at $9.4 \mathrm{GeV}$ at DORIS, and at 13.0 and $17.0 \mathrm{GeV}$ at PETRA by the PLUTO [128 and TASSO [129] collaborations were well described by a theoretical Monte Carlo [130 taking into account the production processes $e^{+} e^{-} \rightarrow c \bar{c}$ and $e^{+} e^{-} \rightarrow b \bar{b}$, with the subsequent non-leptonic decays $c \rightarrow s u \bar{d}$ and $b \rightarrow c \bar{u} d$, following the CKM theory of weak decays.

The effects of heavy quark production and decays above their respective thresholds on the jet distributions are taken into account by a three-step modifications of the light quark pair production and subsequent fragmentation [130. The heavy quark mass enters the Lorentz-invariant density matrix for $e^{+} e^{-} \rightarrow Q \bar{Q}$ (here $Q^{2}=s$ ):

$$
|M|^{2}=\frac{\alpha^{2}}{Q^{4}}\left[\left(\ell_{+} p_{1}\right)\left(\ell_{-} p_{2}\right)+\left(\ell_{+} p_{2}\right)\left(\ell_{-} p_{1}\right)+m_{Q}^{2} Q^{2} / 2\right],
$$

where $\ell_{-}\left(\ell_{+}\right)$is the electron (positron) momentum and $p_{1}\left(p_{2}\right)$ is the momentum of $Q(\bar{Q})$, and the quark mass is denoted by $m_{Q}$. In the second step, the heavy quark 
(antiquark) fragments into a heavy hadron and a number of light hadrons, determined by a function $f_{Q}^{H}(z)$, which peaks increasingly near $z \rightarrow 1$, as $m_{Q}$ increases. In the third step, the heavy hadrons decay, dominantly non-leptonically, modelled on the quark transitions $Q(p) \rightarrow q_{1}\left(q_{1}\right)+\bar{q}_{2}\left(q_{2}\right)+q_{3}\left(q_{3}\right)$ [131. These effects are important quantitatively for jet physics for the lower PETRA energies (typically $\leq 30 \mathrm{GeV}$ ).

\subsection{3-jet events and cross sections at PETRA}

As discussed earlier, the characteristic feature of the process $e^{+} e^{-} \rightarrow q \bar{q}$ with the subsequent fragmentation of the quarks and the antiquarks into a jet of hadrons is that it leads to a two-jet configuration. In QCD, the diagrams shown in Fig. 9 modify this picture. These corrections being proportional to $\alpha_{s}\left(Q^{2}\right)$, the QCD coupling constant at the scale $Q^{2}$, are small. However, the process $e^{+} e^{-} \rightarrow q \bar{q} g$ may reflect itself in a structure of the final states that topologically is different from the dominant process $e^{+} e^{-} \rightarrow q \bar{q}$. The radiated gluon provides a new (non-local) mechanism for producing large- $p_{T}$ hadrons, which, unlike the $p_{T}$ of the hadrons generated in the process $e^{+} e^{-} \rightarrow q \bar{q}$, is expected to increase with the $e^{+} e^{-}$centre-of-mass energy. Thus, broadening of the transverse momentum of the hadrons with increasing centre-of-mass energy is a consequence of gluon bremsstrahlung. It was argued in [30], that a corollary of this phenomenon is that a third jet should exist in the direction of the large $p_{T}$ particle. In particular, if there is enough phase space available, i.e. for large enough $Q$, a three-jet topology in the shape of "Y" (Mercedz-Benz symbol) should emerge, clearly distinguishable from the (dominant) oblate cigar topology corresponding to two-jet events.

The calculation for the process $e^{+} e^{-} \rightarrow q\left(p_{1}\right)+\bar{q}\left(p_{2}\right)+g\left(p_{3}\right)$, shown in the upper two Feynman diagrams in Fig. 9 leads to the following (Dalitz) distribution:

$$
\frac{1}{\sigma_{0}} \frac{d^{2} \sigma}{d x_{1} d x_{2}}=\frac{\alpha_{s}\left(Q^{2}\right)}{2 \pi} C_{F} \frac{x_{1}^{2}+x_{2}^{2}}{\left(1-x_{1}\right)\left(1-x_{2}\right)},
$$

where $Q^{2}=4 E^{2}, x_{i}=E_{i} / E=2 E_{i} / Q$, and $E_{i}$ are the energies of the quark, antiquark, and gluon, with $x_{1}+x_{2}+x_{3}=2$, and $\sigma_{0}$ is the lowest order $e^{+} e^{-} \rightarrow$ hadron cross section given in Eq. (21). The differential cross section in Eq. (35) diverges near the end-points $x_{1,2} \rightarrow 1$, and indeed has infra-red and collinear divergences. We shall discuss finite 2-jet and 3-jet cross sections in the next subsection, but for the present discussion these divergences can be removed by a reasonable cut-off procedure, such as a cut-off $Q_{0}^{2}$ on the invariant masses $s_{13}=Q^{2}\left(1-x_{2}\right)$ and $s_{23}=Q^{2}\left(1-x_{1}\right)$, yielding a finite lowest order three-jet fraction.

\subsection{Experimental evidence of three-jet events at PETRA}

While valuable tests of QCD were performed in studies of the $\Upsilon$ decays, based on the underlying mechanism $\Upsilon \rightarrow 3 g$ and the subsequent fragmentation of the gluons, threejet events were first observed in $e^{+} e^{-}$annihilation at PETRA in 1979 by the four experimental collaborations: TASSO [25], MARK-J [26], PLUTO 27] and JADE [28]. The process $e^{+} e^{-} \rightarrow q \bar{q} g$ leads to planar events, the search of three-jet events in these experiments was concentrated mainly in demonstrating the excess of planar events compared to the estimates based on the 2-jet final states around $\sqrt{s}=27 \mathrm{GeV}$, where most of the early experiments at PETRA were carried out. Such quantitative analyses were backed up by topologically well separated 3-jet events. Fig. 14 shows momentumspace representation of a representative two-jet and three-jet event measured by the TASSO collaboration, analysed on the basis of sphericity tensor and jettiness [40]. 

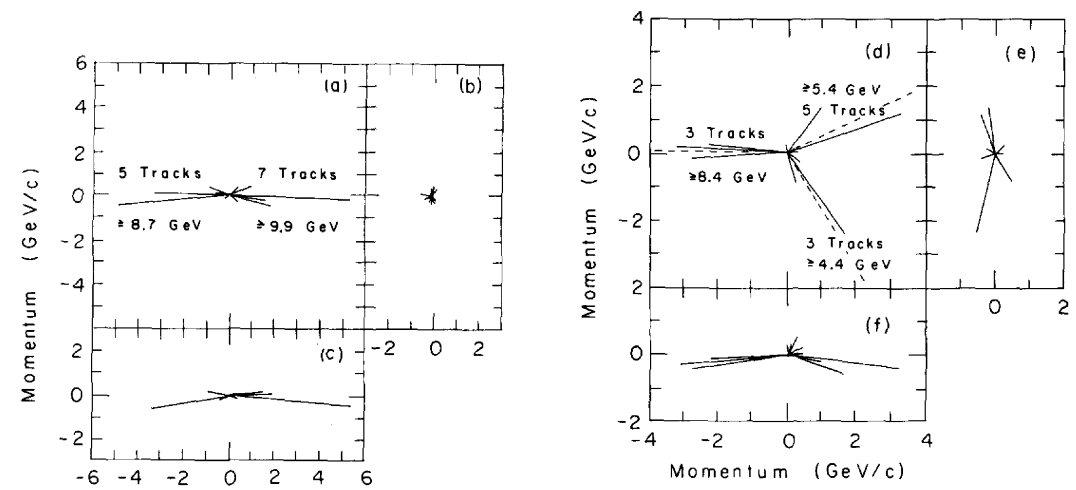

Fig. 14. Momentum space representation of a two-jet event (a) - (c) and a three-jet event (d) - (f) in each of the three projections, (a),(d) $\hat{n}_{2}-\hat{n}_{3}$ plane; (b),(e) $\hat{n}_{1}-\hat{n}_{2}$ plane; (c), (f) $\hat{n}_{1}-\hat{n}_{3}$ plane. Here $\hat{n}_{i}$ are the three axes of the sphericity tensor. (From TASSO [25]).

The MARK-J measurement of the distributions in oblateness (defined below) at $\sqrt{s}=17 \mathrm{GeV}$ and at higher energies $(27.4+30+31) \mathrm{GeV}$ are shown in Fig. 15)(a) and Fig. 15 (b), respectively. For this measurement, the coordinate system is defined by the thrust axis $\mathbf{e}_{\mathbf{1}}$, the major axis $\mathbf{e}_{\mathbf{2}}$, which is in the plane perpendicular to $\mathbf{e}_{\mathbf{1}}$, and is in the direction along which the projected energy in that plane is maximised, and the minor axis, $\mathbf{e}_{\mathbf{3}}$, which is orthogonal to both $\mathbf{e}_{\mathbf{1}}$ and $\mathbf{e}_{\mathbf{2}}$. Oblateness is then defined as

$$
\mathcal{O}=F_{\text {major }}-F_{\text {minor }},
$$

where $F_{\text {major }}=\sum_{i} \mathbf{p}_{\mathbf{i}} \cdot \mathbf{e}_{\mathbf{2}} / \sum_{i}\left|p_{i}\right|$ and $F_{\text {minor }}=\sum_{i} \mathbf{p}_{\mathbf{i}} \cdot \mathbf{e}_{\mathbf{3}} / \sum_{i}\left|p_{i}\right|$. The two frames on the r.h.s. of this figures show the energy flow in the event plane defined by the thrust and major axes (upper frame) and by the thrust and the minor axes (lower frame). These measurements were compared with the $q \bar{q}$ (two-jet) and $q \bar{q} g$ (three-jet) Monte Carlo models [3132, and clearly favoured the $q \bar{q} g$ description, in a statistically significant way.

PLUTO studied the averages of the momenta of the charged particles $\left\langle p_{\|}\right\rangle$, where $p_{\|}$is the longitudinal momentum, $\left\langle p_{\perp}\right\rangle$ and $\left\langle p_{\perp}^{2}\right\rangle$, measured relative to the thrust axis of the event as a function of the c.m. energy. Their analysis showed that the quantities $\left\langle p_{\|}\right\rangle$and $\left\langle p_{\perp}\right\rangle$ are not very discriminative between the $q \bar{q}$ and $q \bar{q} g$, but the energy dependence of $\left\langle p_{\perp}^{2}\right\rangle$ is better described if gluon bremsstrahlung is included. To study this effect in more detail, they distinguished for every event the two jets which are separated by a plane perpendicular to the thrust axis. The jet with the lower (higher) average $\left\langle p_{\perp}\right\rangle$ is called the slim (fat) jet. Fig. 16(a) shows $\left\langle p_{\perp}^{2}\right\rangle$ of the charged particles as a function of the c.m. energy, where the average is taken over the charged hadrons in all slim (fat) jets. For the slim jet the $q \bar{q}$ and $q \bar{q} g$ predictions from the Monte Carlo 31 are very similar and the data are in agreement with both. For the fat jet, however, the data clearly favour $q \bar{q} g$, and $q \bar{q}$ is ruled out. Fig. [16(b) and 16(c) show the so-called "sea-gull plot", obtained by plotting the variable $x_{p}=p / p_{\text {beam }}$ and $\left\langle p_{\perp}^{2}\right\rangle$, at lower c.m. energies 13 and $17 \mathrm{GeV}$ and at higher energies 27.6, 30 and $31.6 \mathrm{GeV}$, respectively. At the lower energy (Fig. 16(b)), there is very little difference between $q \bar{q}$ and $q \bar{q} g$ predictions. For the higher energies(Fig. 16(c)), $q \bar{q} g$ predicts a genuine one-sided jet broadening caused by the gluon jet; the effect is quite dramatic, especially at high $x_{p}$. TASSO collaboration [25] has done a very similar analysis.

The JADE analysis is based on the normalised sphericity tensor $S_{\alpha \beta}$ (defined in Eq. (10) ) and the resulting eigenvalues $Q_{1}, Q_{2}, Q_{3}$ obtained by diagonalising this 

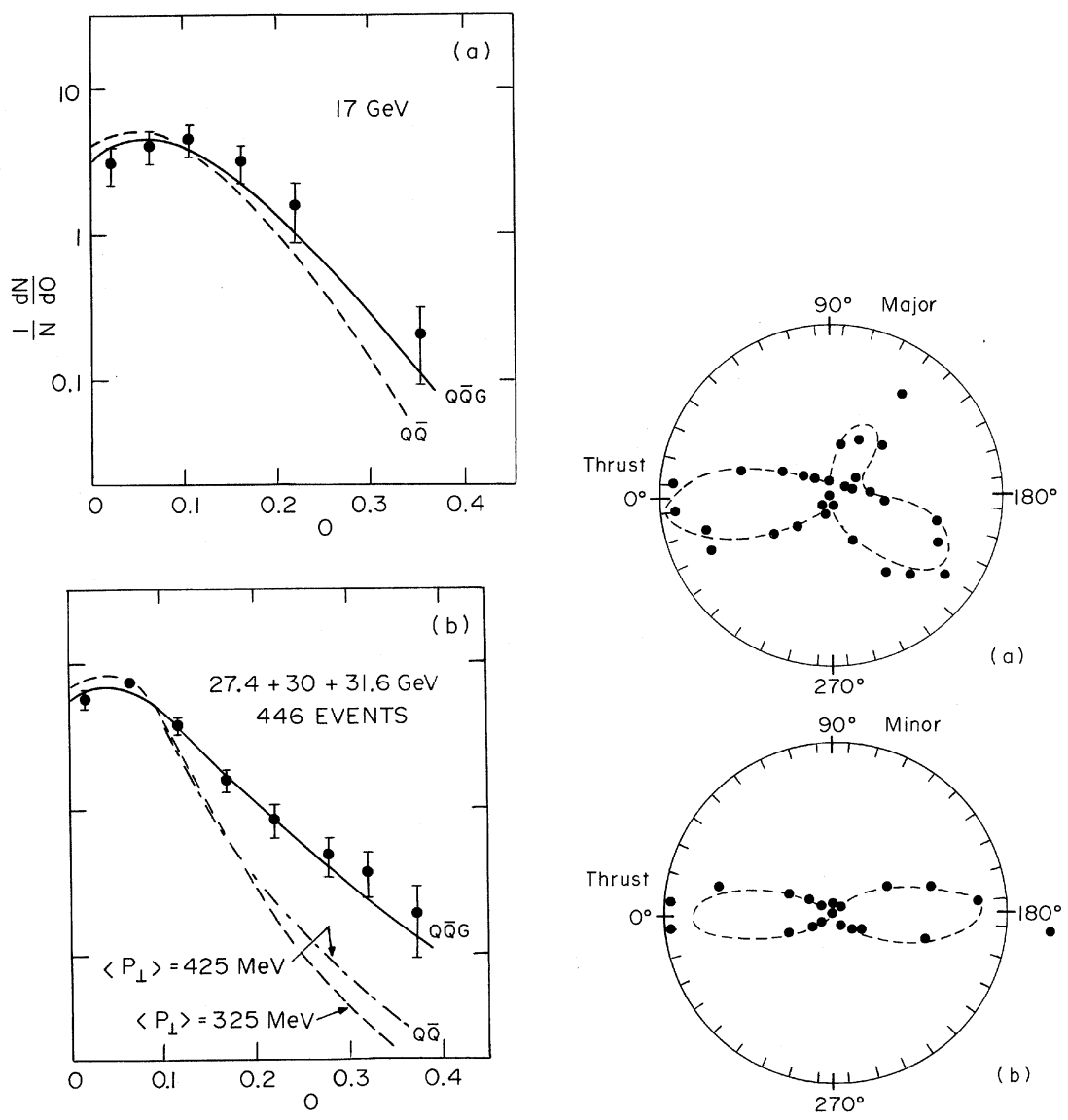

Fig. 15. Left-hand frames: Normalised Oblateness distribution at $\sqrt{s}=17 \mathrm{GeV}$ (a), and at $\sqrt{s}=27.4-31.6 \mathrm{GeV}$ (b). The solid curves are the predictions based on a $Q \bar{Q} g$ model and the dashed curves are based on the $Q \bar{Q}$ model with $\left\langle p_{T}\right\rangle=325 \mathrm{MeV}$ (denoted as $q \bar{q} g$ and $q \bar{q}$, respectively, in this review). The dashed-dotted curve in (b) is the $Q \bar{Q}$ model prediction with $\left\langle p_{T}\right\rangle=425 \mathrm{MeV}(Q=u, d, s, c, b)$. Right-hand frames: Energy flow in the event plane defined by (a) the thrust and the major axes, and (b) by the thrust and the minor axes with the events satisfying the cuts thrust $<0.8$ and oblateness $>0.1$ at $\sqrt{s}=27.4-31.6$ $\mathrm{GeV}$. The energy value is proportional to the radial distances.; dots are the experimental measurements (From MARK-J [26]).

tensor on an event by event basis. The variables which play a central role in this analysis are the sphericity $=3 / 2\left(Q_{1}+Q_{2}\right)$ and planarity $=\left(Q_{2}-Q_{1}\right)$. Fig. [17 shows the planarity distribution $d N / d\left(Q_{2}-Q_{1}\right)$ measured by JADE at $\sqrt{s}=27.7$ and 30 $\mathrm{GeV}$. Their data are compared with a $q \bar{q}$ model, with $\sigma_{q}=250 \mathrm{MeV}$ and $350 \mathrm{MeV}$, both of which fail to describe the data. The $q \bar{q} g$ model describes the data well.

The results reviewed in this section were the first measurements through which the effect of a third (gluon) jet was convincingly established in $e^{+} e^{-}$annihilation. This is an important milestone in the confirmation of QCD in which jet physics played a central role. From a theoretical point of view, observation of the gluon jet was inevitable. Like many other discoveries in particle physics, this discovery needed high energy $e^{+} e^{-}$beams, particle detectors well equipped to measure the characteristics of the hadrons, and data analysis techniques. This was the work of dedicated teams 


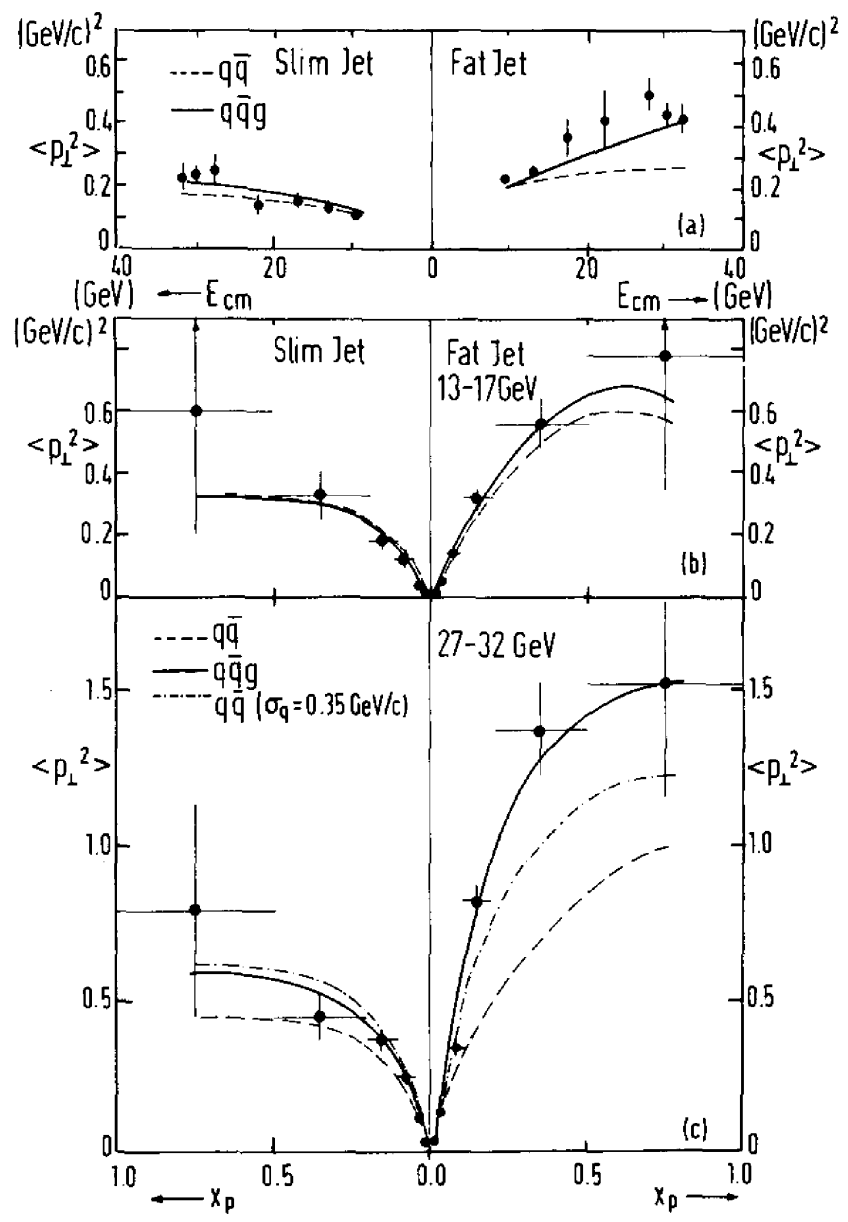

Fig. 16. Average observed $\left\langle p_{T}^{2}\right\rangle$ of charged particles in the slim and fat jets as a function of the c.m. energy (a). Sea-gull plots $\left(\left\langle p_{T}^{2}\right\rangle\right.$ as a function of $x_{p}=p / p_{\text {beam }}$, where $\left.p=\sqrt{p_{\|}^{2}+p_{T}^{2}}\right)$ for slim and fat jets in two separate energy range (b), (c). The solid and dashed curves are $q \bar{q} g$ and $q \bar{q}$ predictions, respectively. In (c), the dashed curve corresponds to $\sigma_{q}=0.247 \mathrm{GeV}$ (default value) and the dash-dotted curve to $\sigma_{q}=0.35 \mathrm{GeV}$. (From PLUTO [27]).

of machine builders and experimental physicists who should be credited with the discovery. For the interested readers we refer to individual accounts leading to the discovery of the gluon jets [112 132 133/134/135]136/137, but stress that this list of references is by no means exhaustive.

\subsection{Quantitative studies of QCD at PETRA and PEP}

Subsequent to the discovery of the gluon jet, the four PETRA collaborations, JADE, Mark-J, PLUTO (later replaced by CELLO) and TASSO collaborations made many more measurements in $e^{+} e^{-}$annihilation to hadrons, in which further evidence for the gluon jet was presented, These prompted quantitative studies of QCD for inclusive jet-observables, like thrust and the Fox-Wolfram shape variable [138 etc., and for jet topology, like the 2 -jet and 3 -jet rates etc. Also the gluon spin was deter- 


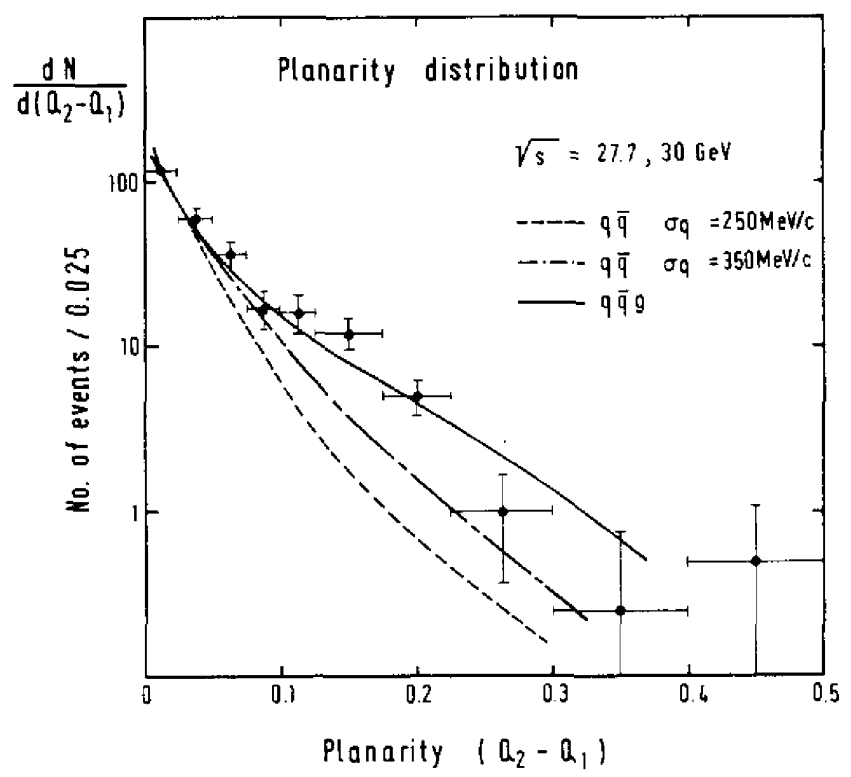

Fig. 17. The planarity distribution compared with the model predictions for $e^{+} e^{-} \rightarrow q \bar{q}$ and $e^{+} e^{-} \rightarrow q \bar{q} g$ at $\sqrt{s}=27.7,30 \mathrm{GeV}$. (From JADE [28]).

mined, following a suggestion in [139. One important issue was the universality of the quark-gluon coupling $\alpha_{s}\left(Q^{2}\right)$, i.e. to check whether the same value is obtained independent of the observables and the measurements of $\alpha_{s}\left(Q^{2}\right)$ at various values of $s=Q^{2}$ were consistent with the evolution anticipated by the renormalisation group. These attempts to obtain $\alpha_{s}\left(Q^{2}\right)$ required the calculation of next-to-leading order corrections to the topological jet-rates and inclusive jet-observables, and also required a better understanding of the non-perturbative models used to interpret the experimental data. These theoretical and phenomenological studies often took the form of detailed Monte Carlo programs without which no realistic comparison of theory and experiment was possible. In fact, since the days of experimentation at PETRA and PEP, Monte Carlo based theoretical frameworks have become indispensable for the quantitative analysis of data, as witnessed later at LEP, HERA and the Tevatron, and now at the LHC.

In $O\left(\alpha_{s}\left(Q^{2}\right), 2\right.$-jet cross sections defined by a jet-resolution criterion, such as the Sterman-Weinberg jet-cones or the jet invariant mass, receive contributions from the virtual corrections to the process $e^{+} e^{-} \rightarrow q \bar{q}$, and soft or collinear configurations from the processes $e^{+} e^{-} \rightarrow q \bar{q} g$. In $O\left(\alpha_{s}^{2}\left(Q^{2}\right)\right)$, the 3 -jet cross sections receive contribution from the virtual corrections to $e^{+} e^{-} \rightarrow q \bar{q} g$ and soft and collinear configurations from the 4-parton processes $e^{+} e^{-} \rightarrow q \bar{q} g g$ and $e^{+} e^{-} \rightarrow q \bar{q} q \bar{q}$. The hard and non-collinear configurations in the 4-parton processes give rise to 4 -jet cross sections, with the leading contribution arising in $O\left(\alpha_{s}^{2}\left(Q^{2}\right)\right)$, whose rates were calculated in 140141] including the quark mass effects. They were important to check the non-abelian character of QCD, as discussed later. The first complete next-to-leading order correction to event shapes up to order $\alpha_{s}^{2}$ were undertaken by Ellis et al. 142 143. They presented their results in terms of the tensor

$$
\left.\theta^{i j}=\sum_{a}\left(p_{a}^{i} p_{a}^{j}\right) /\left|p_{a}\right|\right)\left(\sum_{a}\left|p_{a}\right|\right)^{-1}
$$


where $p_{a}^{i}$ are the components of the centre-of-mass three-momentum of hadrons $a$, and the sum runs over all hadrons. The eigenvalues of $\theta$ are determined by the characteristic equation

$$
\lambda^{3}-\lambda^{2}+\frac{1}{3} C \lambda-D / 27=0, \quad 0 \leq C, D \leq 1 .
$$

The quantities $C$ (also called the Fox-Wolfram shape variable [138]) and $D$ are symmetric functions of the eigenvalues, defined as

$$
C \equiv 3\left(\lambda_{1} \lambda_{2}+\lambda_{2} \lambda_{3}+\lambda_{3} \lambda_{1}\right), \quad D=27 \lambda_{1} \lambda_{2} \lambda_{3},
$$

Integrating $\frac{1}{\sigma} \frac{d \sigma}{d C}$ in the range $\frac{1}{2}<C<1$ yields

$$
\frac{1}{\sigma_{0}} \int_{0.5}^{1.0} d C \frac{d \sigma}{d C}=C_{1} \frac{\alpha_{s}\left(Q^{2}\right)}{\pi}\left(1+C_{2} \frac{\alpha_{s}\left(Q^{2}\right)}{\pi}\right) \text {. }
$$

Numerically, $C_{1}=2.8$ and $C_{2}=18.2 \pm 0.7$ for five quark flavours 142 143. Thus, large corrections are obtained for the Fox-Wolfram shape variable, $C$.

Another, and experimentally widely studied, example of an inclusive distribution is thrust. In $O\left(\alpha_{s}^{2}\right)$, this was first calculated by Vermaseren et al. [144, and verified subsequently by Ellis and Ross [145] and by others [146147148, using the earlier work reported in [142143, In next-to-leading order NLO in $\alpha_{s}$, thrust-distribution in $e^{+} e^{-} \rightarrow$ hadrons is given by the following expression

$$
\frac{d \sigma}{d T}=A_{0}(T) \frac{\alpha_{s}\left(Q^{2}\right)}{\pi}+A_{1}(T)\left(\frac{\alpha_{s}\left(Q^{2}\right)}{\pi}\right)^{2},
$$

where the functions $A_{0}(T)$ and $A_{1}(T)$ are shown in Fig. 18 (note that the variable $t$ used in these plots is the same as $T$ used in the text). The shapes of $A_{0}(T)$ and $A_{1}(T)$ are rather similar, but the $O\left(\alpha_{s}^{2}\left(Q^{2}\right)\right.$ corrections to the thrust-distributions are also numerically large. Integrating the distribution in Eq. (41) up to $T=0.85$ yields

$$
\frac{1}{\sigma_{0}} \int_{0.5}^{0.85} d T \frac{d \sigma}{d T}=K_{1} \frac{\alpha_{s}\left(Q^{2}\right)}{\pi}\left(1+K_{2} \frac{\alpha_{s}\left(Q^{2}\right)}{\pi}\right) .
$$

Numerically, $K_{1}=1.156, K_{2}=17.6 \pm 0.3$ for five quark flavours, which for $\alpha_{s}\left(Q^{2}\right)=$ 0.13 at $\sqrt{s}=35 \mathrm{GeV}$ yields a correction of about $70 \%$. Theoretical calculations from [142] were later implemented in the independent jet Monte Carlo 32 and used to determine $\alpha_{s}\left(Q^{2}\right)$ from the inclusive measurements. The first such determination using the thrust and oblateness distributions, measured by the TASSO 149 and MARK-J [150] collaborations, respectively, yielded [148] $\alpha_{s}(Q=35 \mathrm{GeV})=0.128 \pm$ 0.013 from the TASSO data and $\alpha_{s}(Q=35 \mathrm{GeV})=0.120 \pm 0.010$ from the MARK-J data.

Subsequently, an enormous effort has gone into estimating the effects from the jet resolutions, choice of jet variables, and fragmentation models. Also, the statistical significance of the data from the experiments at PETRA and PEP increased enormously over the time. An observable studied intensively in theory and experiments at PETRA is the energy-energy correlation (EEC) and its asymmetry (AEEC). EEC is a measure of the energy flow involving two calorimeters subtending solid angles $\Omega$ and $\Omega^{\prime}$ with respect to the incoming $e^{+} e^{-}$axis. Keeping the orientation between the two calorimeter cells fixed $(=\chi)$, the differential distribution in $\cos \chi$ can be expressed as

$$
\frac{1}{\sigma} \frac{d \Sigma^{E E C}}{d \cos \chi}=\frac{1}{\sigma} \sum \int \frac{d \sigma}{d x_{i} d x_{j} d \cos \chi} x_{i} x_{j} d x_{i} d x_{j},
$$




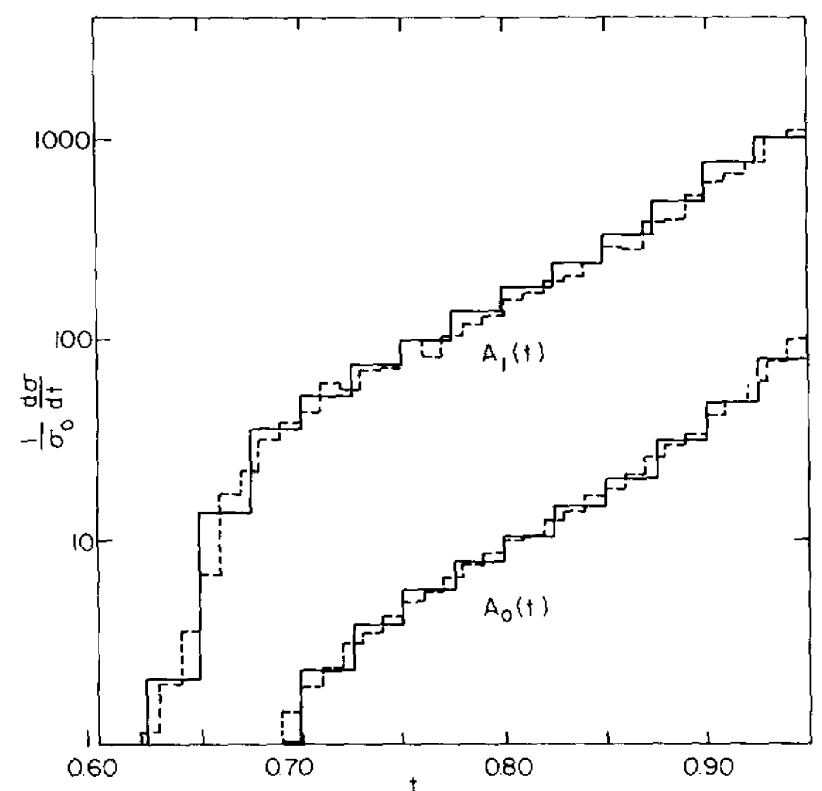

Fig. 18. The scalar functions $A_{0}(t)$ and $A_{1}(t)$ for the Thrust distribution as defined in Eq.41 Solid histograms (based on Ellis, Ross and Terrano [142]), dashed histograms (Vermaseren et al. 144]). (From [145]).

where $x_{i}$ are the scaled energies in terms of the c.m. energy $\sqrt{s}$. The experimental configurations with fixed angle between the calorimeters $\chi$ and the polar angle of one of the calorimeters $\theta$ are calculable in perturbative QCD 151152. However, most experimental measurements were carried out for the averaged EEC, obtained by integrating over $\cos \theta$, for which perturbative QCD yields the following expression (for $m_{q}=0$ )

$$
\frac{1}{\sigma_{0}} \frac{d \Sigma^{E E C}}{d \cos \chi}=\frac{\alpha_{s}\left(Q^{2}\right)}{\pi} F(\xi),
$$

where $\xi=\frac{1-\cos \chi}{2}$ and $F(\xi)$ is given by 151152

$$
F(\xi)=\frac{(3-2 \xi)}{6 \xi^{2}(1-\xi)}\left[2\left(3-6 \xi+2 \xi^{2}\right)+\ln (1-\xi)+3 \xi(2-3 \xi)\right]
$$

The averaged (obtained by integrating over $\cos \theta$ ) AEEC cross section has an obvious definition

$$
\begin{aligned}
\frac{1}{\sigma_{0}} \frac{d \Sigma^{A E E C}}{d \cos \chi} & \equiv \frac{1}{\sigma_{0}} \frac{d \Sigma^{E E C}(\pi-\chi)}{d \cos \chi}-\frac{1}{\sigma_{0}} \frac{d \Sigma^{E E C}(\chi)}{d \cos \chi} \\
& =\frac{\alpha_{s}\left(Q^{2}\right)}{\pi}\left[(F(1-\xi)-F(\xi)] \equiv \frac{\alpha_{s}\left(Q^{2}\right)}{\pi} A(\xi) .\right.
\end{aligned}
$$

Effects of quark masses in the EEC and AEEC cross sections were calculated in 153154155156]. The $O\left(\alpha_{s}^{2}\left(Q^{2}\right)\right.$ corrections to these distributions were calculated numerically [153 154 157/158]. Restricting the angular range to $-0.95<\cos \chi<0.95$, where the non-perturbative effects are relatively small, the NLO corrections to the EEC cross-section were found 

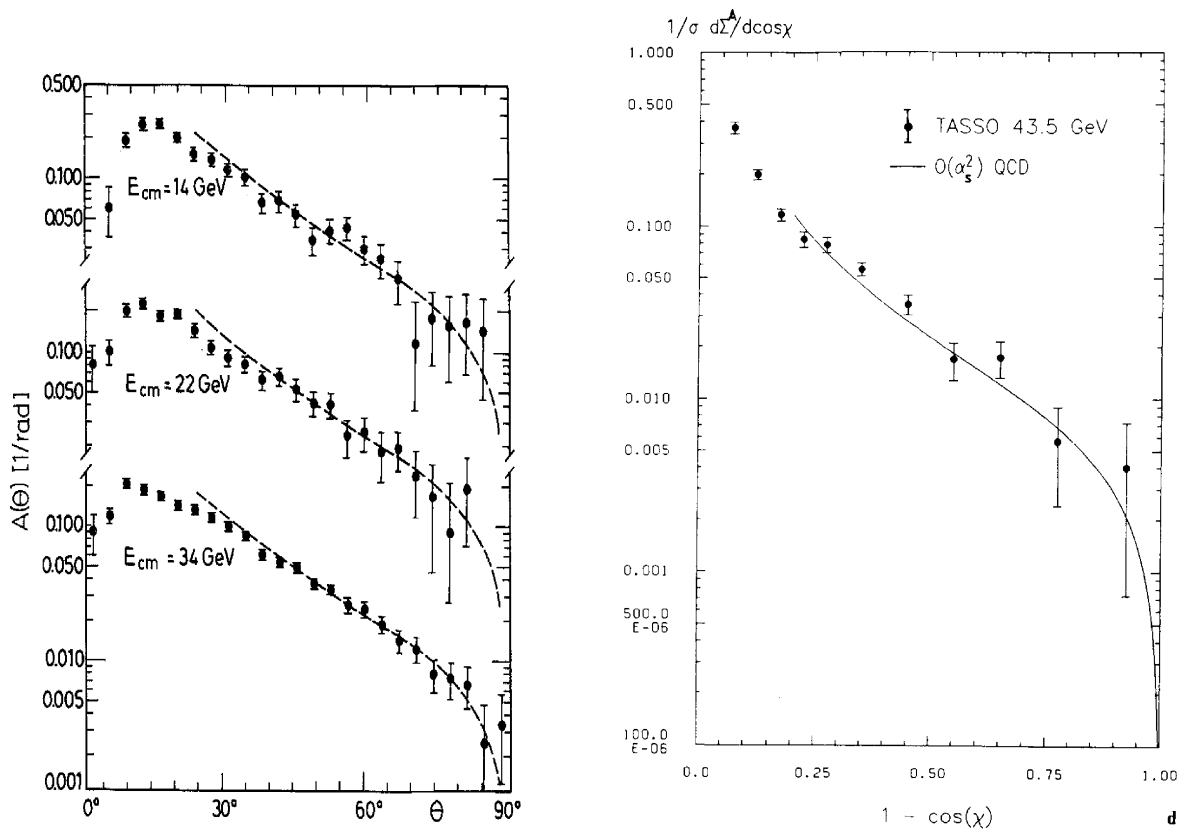

Fig. 19. The asymmetric part of the energy energy correlation cross section measured by the JADE [159] and [TASSO] 161. collaborations at PETRA and comparison with the perturbative QCD calculations including $O\left(\alpha_{s}^{2}\left(Q^{2}\right)\right)$ corrections from 153 . The distribution in the upper left-hand frame from the JADE collaboration shows the corrected asymmetry $A(\theta)$ vs. $\theta$, measured at $\sqrt{s}=14,22$ and $34 \mathrm{GeV}$. The upper right-hand frame from TASSO shows the measurements at $\sqrt{s}=43.5 \mathrm{GeV}$ and comparison with the perturbative QCD (solid curve).

to be moderate, typically $O(35 \%)$, but the corresponding corrections to the AEEC were small, typically $O(10 \%)$, giving reasons to be optimistic about the convergence of perturbative QCD in these variables, particularly the AEEC.

Measurements of the EEC and AEEC were undertaken by all four experiments at PETRA: JADE, MARK-J, TASSO, and PLUTO. The AEEC measurements have been used to determine $\alpha_{s}\left(Q^{2}\right)$ by comparing them with the NLO expression 153 . The extracted values of $\alpha_{s}\left(Q^{2}\right)$ are found to be: $\alpha_{s}\left(Q^{2}=(34 \mathrm{GeV})^{2}\right)=0.115 \pm$ $0.005[\mathrm{JADE}][159], \alpha_{s}\left(Q^{2}=(34 \mathrm{GeV})^{2}=0.13[\mathrm{MARK}-\mathrm{J}][160], \alpha_{s}\left(Q^{2}=(34.8 \mathrm{GeV})^{2}\right)\right.$ $=0.125 \pm 0.005$ [TASSO] [161], and $\alpha_{s}\left(Q^{2}=(34.6 \mathrm{GeV})^{2}\right)=0.125 \pm 0.005$ [PLUTO] [162. Within errors, these values of $\alpha_{s}\left(Q^{2}\right)$ are consistent with each other, and with the ones from oblateness and thrust distributions, given earlier. Representative distributions from the JADE [159] and [TASSO] [161] collaborations are shown in Fig. 19, in which $A(\theta)$ vs. $\theta$ and $1 / \sigma d \Sigma^{\mathrm{A}} / d \cos \chi$ vs. $1-\cos \chi$ are plotted, respectively. These measurements are compared with the perturbative QCD expression, calculated to $O\left(\alpha_{s}^{2}\left(Q^{2}\right)\right)$ and the agreement is impressive for $(\theta, \chi)>30^{\circ}$. For $(\theta, \chi)<30^{\circ}$, one needs to implement non-perturbative effects as well as the resummation of the large $\operatorname{logs}$ to all orders in perturbation theory.

A lot of experimental effort went also in studying the topological cross sections (jet multiplicity) in $e^{+} e^{-}$annihilation experiments at PETRA, PEP, TRISTAN and later at LEP. Theoretical distributions to these topologies were calculated in a series of papers 93|163164|165|166|167. Making use of this theoretical work, the JADE collaboration measured $\alpha_{s}\left(Q^{2}\right)$ in a limited range of $\sqrt{s}$ using the three-jet rate and estab- 
lished the running of $\alpha_{s}\left(Q^{2}\right)$. Defining the fractional three-jet rate $R_{3}=\sigma_{3-\text { jet }} / \sigma_{\text {tot }}$ as a function of $y_{\min }$, which is a cut-off parameter such that $y_{i j} \geq y_{\min }$ for any pair of partons $i$ and $j$ and $y_{i j}=M_{i j}^{2} / s$, the measured jet-rate was fitted to the expression

$$
R_{3}\left(y_{\min }\right)=C_{1} \alpha_{s}\left(Q^{2}\right)+C_{2} \alpha_{s}^{2}\left(Q^{2}\right)
$$

where $C_{1}$ and $C_{2}$ are $y_{\text {min }}$-dependent constants calculated by Kramer and Lampe (called KL below) in [166. The JADE measurements for $R_{3}\left(y_{\min }\right)$ as a function of $\sqrt{s}$ in the range $20<\sqrt{s}<44 \mathrm{GeV}$ are shown in Fig. 20 (left-hand frame) [168. They follow nicely the RG-prescribed running of $\alpha_{s}\left(Q^{2}\right)$ with $\Lambda_{\overline{M S}}=205 \mathrm{MeV}$ for $0.04<y_{\min }<0.12$ using $\mathrm{KL}$, with almost the same value $\Lambda_{\overline{M S}}=210 \mathrm{MeV}$ using a calculation by Gottschalk and Shatz (called GS) [169]. An even more convincing measurement of the running of $\alpha_{s}\left(Q^{2}\right)$ was presented by the MARK II collaboration [170] at PEP and SLC. They determined $\alpha_{s}\left(Q^{2}\right)$ from the differential three-jet rate $\left.g_{3}\left(y_{3}\right)\right|_{y_{3}=y_{\text {cut }}}=\frac{\partial}{\partial y_{\text {cut }}} f_{2}\left(y_{\text {cut }}\right)$, where $f_{2}\left(y_{\text {cut }}\right)$ is the fraction of two-jet events defined by the jet resolution $y_{\text {cut }}$. Their result for $g_{3}\left(y_{3}\right)$ is shown as a function of $y_{3}$ in Fig. 20 (right-hand frame) for two values $\sqrt{s}=91 \mathrm{GeV}$ (SLC) and $\sqrt{s}=29 \mathrm{GeV}$ (PEP). The three curves shown are the predictions of KL [166] for three different values $\Lambda_{\overline{M S}}=0.1,0.3$ and $0.5 \mathrm{GeV}$. Here $\Lambda_{\overline{M S}}$ refers to the QCD scale parameter in a specific renormalisation scheme, the so-called modified minimal subtraction scheme $\overline{M S}[171$. For further reading of the technical issues of renormalisation and schemdependencies at a nonspecialist level, we refer to a review on perturbative QCD [172]. These measurements yielded $\alpha_{s}\left(Q^{2}\right)=0.123 \pm 0.009 \pm 0.005$ at $Q=\sqrt{s}=91 \mathrm{GeV}$ and $\alpha_{s}\left(Q^{2}\right)=0.149 \pm 0.002 \pm 0.007$ at $Q=\sqrt{s}=29 \mathrm{GeV}$, The running of $\alpha_{s}\left(Q^{2}\right)$ is clearly established. A comparison with the values of $\alpha_{s}\left(Q^{2}\right)$ determined from the measurements of the AEEC cross section at PETRA energies, discussed earlier, also shows that non-perturbative effects at these energies are observable dependent and not negligible.

These investigations were extended to jet rates of higher multiplicity, i.e. four-jet and five-jet. An earlier paper along these lines is due to the JADE collaboration, in which $n$-jet rates $(n=2,3,4,5)$ were presented [94. At this time, NLO corrections to the 4-jet rates and even LO predictions for the 5-jet final states did not exist. The data were compared with the leading-logarithmic- approximation (LLA) model . Similar studies based on the MARK II data at PEP $(\sqrt{s}=29 \mathrm{GeV})$ are found in 173 and [174] using the so-called "optimised perturbation theory", i.e., by fitting the scale.

An earlier attempt to establish the non-abelian nature of QCD from a study of multijet events was made by the AMY collaboration at the TRISTAN $e^{+} e^{-}$storage ring at the KEK laboratory 175. Their data showed a clear preference for QCD in contrast to an abelian model. In addition, they showed the running of $\alpha_{s}\left(Q^{2}\right)$ by measuring the 3 -jet rate $R_{3}$ at $\sqrt{s}=50$ to $57 \mathrm{GeV}$ by comparing their measurements with those of the JADE collaboration [168] and the TASSO collaboration [176] at PETRA taken at lower c.m. energies. Other publications towards a determination of $\alpha_{s}$ from PEP and PETRA are for example by MARK II [177] and CELLO [178].

\subsection{String- and String-like effects in Jets}

The data taken by the experimental collaborations at PEP and PETRA have been used also to investigate non-perturbative effects in the jet profiles with the view of testing various phenomenological models available in the 1980's. This was important, since depending on the observables considered, non-perturbative effects influenced also the measurement of $\alpha_{s}$. Several groups [179,180,181,182] have used three-jet 

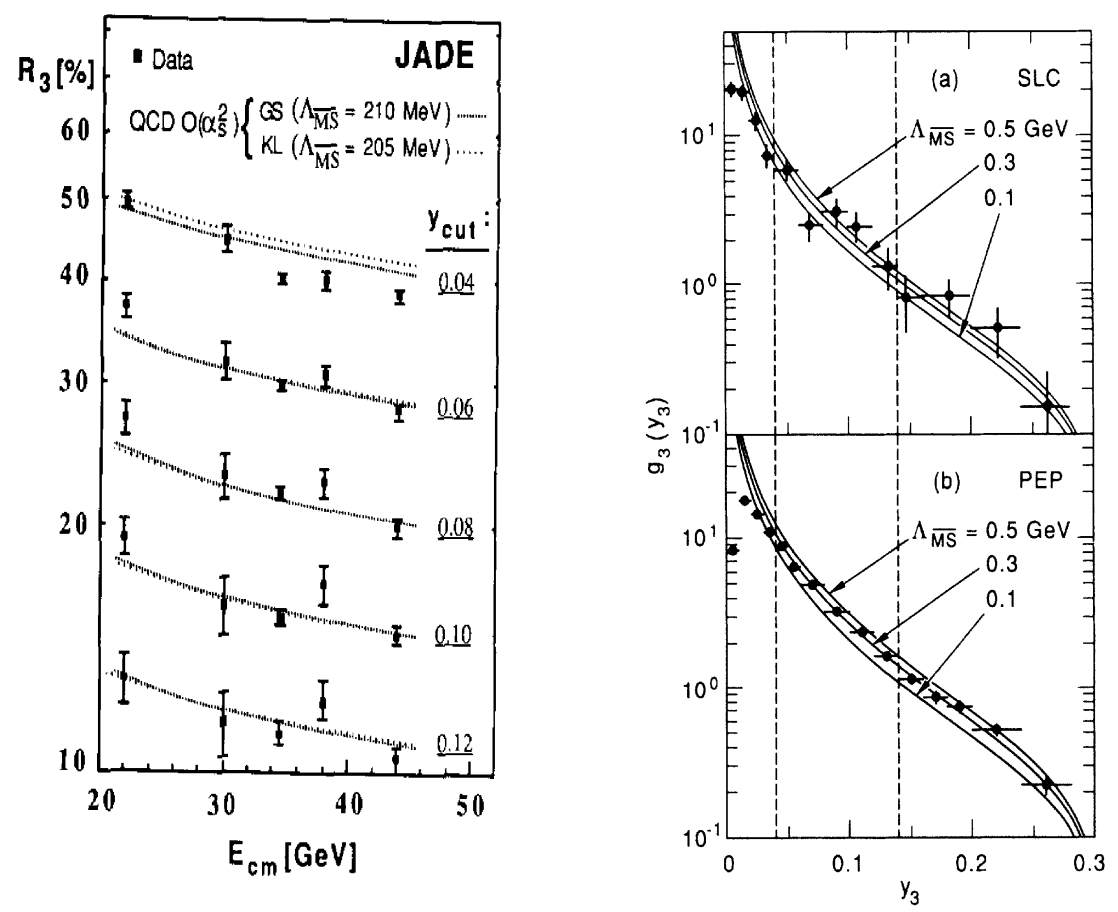

Fig. 20. Left-hand frame: Three-jet event rates measured by the JADE collaboration 168 as a function of the c.m. energy $E_{\mathrm{cm}}[\mathrm{GeV}]$ for the indicated values of the jet resolution parameter $y_{\text {cut }}$, together with the predictions of the order $\alpha_{s}^{2}$ perturbatve calculations by Gottschalk and Shatz (GS) and Kramer and Lampe (KL). Right-hand frame: Experimental distribution $g_{3}\left(y_{3}\right)$ as a function of $y_{3}$ at (a) $\sqrt{s}=91 \mathrm{GeV}$ and $\sqrt{s}=29 \mathrm{GeV}$ measured by the MARKII collaboration [170. The $y_{3}$ range used in the fit for the determination of $\alpha_{s}$ is defined by the two dashed lines. The curves are second order perturbative calculations with the indicated values of $\Lambda \overline{\overline{M S}}$.

$(q \bar{q} g)$ events to study the impact of hard gluon bremsstrahlung on the hadronisation process. In these studies they observed the so-called string effect [47, predicting a depletion of particles in the angular region between the quark and antiquark jet relative to the particle flow in the regions between the quark and gluon jets and the antiquark and gluon jets. In Fig. 21 (left-hand frames), we show the measurements of the normalised energy flow $(1 / E) d E / d \theta$ in planar three-jet events and the normalised charged particle flow in these events undertaken by the JADE collaboration [180 between $\sqrt{s}=30 \mathrm{GeV}$ and $36 \mathrm{GeV}$ at PETRA. These distributions allowed one to distinguish between a hadronisation model [31] in which the fragmentation proceeds along the parton momenta (the independent jet IJ model) and the model in which the fragmentation takes place along the colour-anticolour axes (the LUND string model [38), discussed earlier. Only the leading order $\left(O\left(\alpha_{s}\right)\right)$ matrix elements were taken into account for the gluon bremsstrahlung process $\left(e^{+} e^{-} \rightarrow q \bar{q} g\right)$, which were encoded in these fragmentation models. As seen in this figure, JADE data on the energy and charged particle flow are better reproduced by fragmentation along the colour axes [38.

A similar analysis was undertaken somewhat later in 1985 by the TASSO collaboration 182. In this case, the three-jet events produced in $e^{+} e^{-}$annihilation into hadrons at $34.4 \mathrm{GeV}$ were compared with the $O\left(\alpha_{s}^{2}\right)$ perturbative QCD calculations 

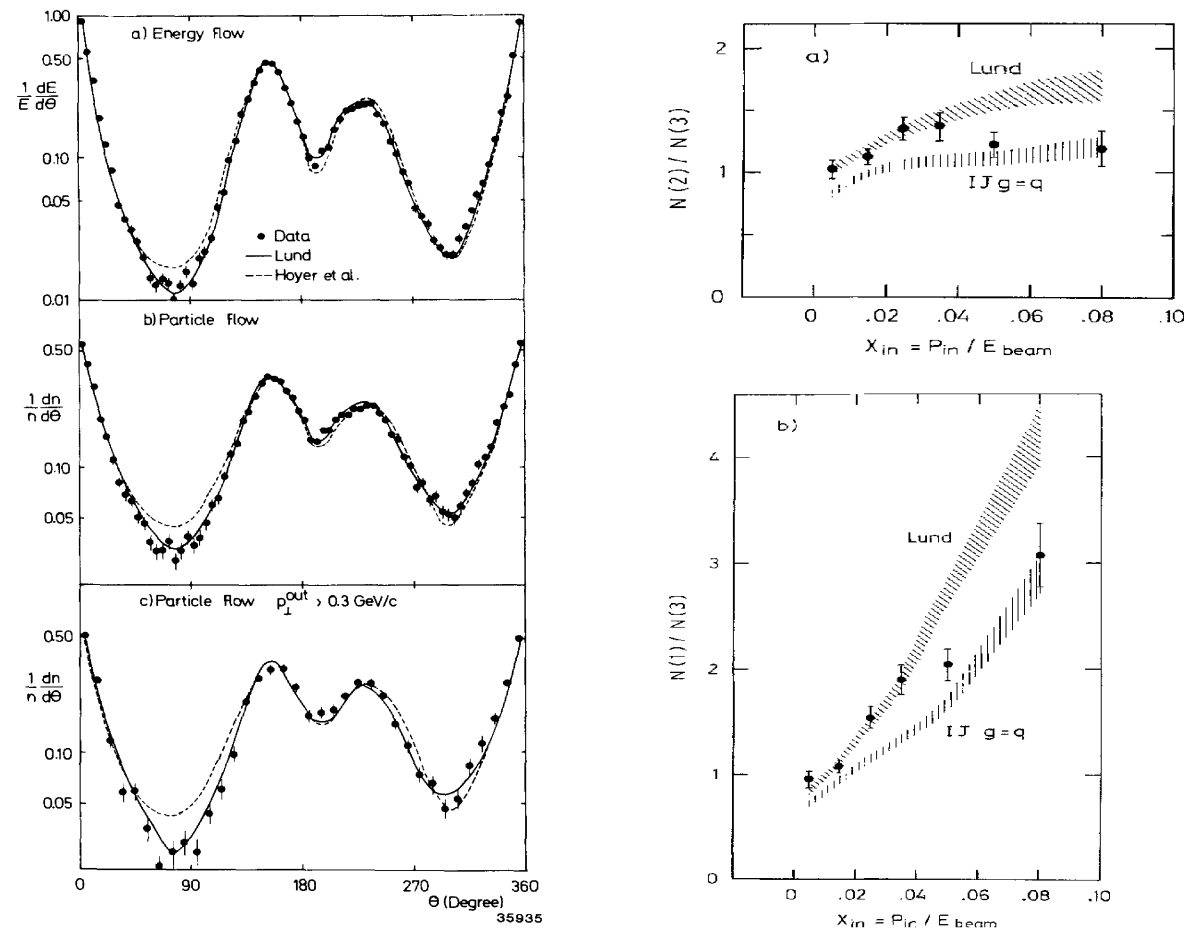

Fig. 21. Left-hand frames: (a) The normalised energy flow $1 / E d E / d \theta$ in the three-jet events compared with two model predictions. (b) The normalised charged particle flow $1 / n d n / d \theta$. (c) $1 / n d n / d \theta$ with $p_{T}^{\text {out }}>0.3 \mathrm{GeV}$. Here $n$ is the total number of particles used in each plot (JADE collaboration [180). Right-hand frames: Ratios of particle densities in the angular gaps between the jet axes, defined by $0.25<\psi_{j}^{\prime}<0.75$ as a function of $x_{\mathrm{in}}$. The calculation of the IJ $(g=q)$ and of the LUND models are shown as shaded bands. a) $N(2) / N(3)$ and b) $N(1) / N(3)$ for the three-jet event sample (TASSO collaboration [182]).

convoluted with two different models of fragmentation (IJ and Lund). The analysis was undertaken in terms of the "reduced azimuthal angles" $\psi_{j}^{\prime}$ and $x_{\text {in }}=p_{\text {in }} / E_{\text {beam }}$, where $p_{\text {in }}$ is the particle momentum projected into the event plane. The $\psi_{j}^{\prime}$ are defined as

$$
\psi_{j}^{\prime}=\frac{\psi-\Phi_{i}}{\Phi_{k}-\Phi_{i}}, i, j, k=1,2,3 \text { and cyclic },
$$

where the particle under consideration is located between jets $i$ and $k\left(\Phi_{i}<\psi<\Phi_{k}\right)$. The reduced angles $\psi_{j}^{\prime}$ run from 0 and 1 . The subscript $j$ denotes the angular region opposite to the jet $j$. The analysis was restricted to $x_{\text {in }}<0.1$ and the data were divided in two samples $x_{\text {in }}<0.04$ and $0.04<x_{\text {in }}<0.1$. The result of the TASSO analysis is displayed in Fig. 21 (right-hand frames) showing that the distribution of low energy (soft) hadrons in the 3 -jet plane is better described by the LUND colour fragmentation model than by the independent jet model. The opposite is true for more energetic particles flowing between the 3 jets.

The "string effect" was subsequently attributed to the coherence of soft gluon emission from the $q \bar{q} g$ system - a characteristic feature of the non-abelian nature of QCD [4]. This is illustrated by contrasting the case of a soft gluon emission (assumed here as $\left.g\left(p_{2}\right)\right)$ in $e^{+} e^{-} \rightarrow q\left(p_{+}\right)+\bar{q}\left(p_{-}\right)+g\left(p_{1}\right)+g\left(p_{2}\right)$ from the process in which the gluon $g\left(p_{1}\right)$ is replaced by a photon, i.e., $e^{+} e^{-} \rightarrow q\left(p_{+}\right)+\bar{q}\left(p_{-}\right)+\gamma\left(p_{1}\right)+g\left(p_{2}\right)$. The angular distribution of the soft gluon (antenna pattern)in the case of $e^{+} e^{-} \rightarrow q \bar{q} \gamma$ is 
given by

$$
W_{+-}\left(\phi_{2}\right) \equiv 2 C_{F} a_{+-} V(\alpha, \beta)=\frac{4 C_{F} a_{+-}}{\cos \alpha-\cos \beta}\left(\frac{\pi-\alpha}{\sin \alpha}-\frac{\pi-\beta}{\sin \beta}\right),
$$

where $\alpha=\phi_{2}$ and $\beta=\theta_{+-}-\phi_{2}$ (see the kinematics shown in the upper left-hand frame in Fig. 22); $a_{i k}=1-\left(\mathbf{n}_{i} \cdot \mathbf{n}_{k}\right)$, with $\mathbf{n}_{i}$ being the unit vector in the direction of $\mathbf{p}_{i}$, and $\theta_{+-}$is the angle between the $q$ and $\bar{q}$ directions. Replacing $\gamma\left(p_{1}\right)$ with a gluon $g\left(p_{1}\right)$ changes the angular distribution essentially due to the antenna element $g\left(p_{1}\right)$ participating in the emission as well. One now obtains $\left(\gamma=\theta_{+1}+\phi_{2}\right)$ :

$$
W_{ \pm 1}\left(\phi_{2}\right)=N_{c}\left[a_{+1} V(\alpha, \gamma)+a_{1-} V(\alpha, \gamma)\right]+\left(2 C_{F}-N_{c}\right) a_{+-} V(\alpha, \beta) .
$$

The (soft) particle flow according to these two configurations is illustrated in Fig. 22 (upper right-side frame) showing that the flow opposite to the direction of $\mathbf{n}_{1}$ is appreciably lower for the case of a gluon than for a photon due to the destructive interference in the case of QCD $(q \bar{q} g g)$.

This phenomenon can be qualitatively understood. Omitting the small contribution from the second terms in Eq. (50), one reduces this equation to the sum of two independent quark antennas $(+, 1)$ and $(-, 1)$. Therefore, in this approximation, the total particle flow can be obtained by the simple incoherent composition of two "annihilations" $e^{+} e^{-} \rightarrow q \bar{q}$, boosted from their respective rest frames to the overall $q \bar{q} g$ c.m. frame. It is clear that the angular region between the $q$ and $\bar{q}$ will be depopulated as it is opposite to the boost direction of both two-jet configurations. This perturbation theory based scenario $(3=2+2+$ Lorentz boost $)$ then coincides with the fragmentation of the gluon in the process $e^{+} e^{-} \rightarrow q \bar{q} g$ events in the LUND fragmentation model. The independent jet model misses this, as the gluon fragments independently on its own. Consequently, the Lorentz boost effect is absent.

The colour coherence study of $e^{+} e^{-}$jets by Azimov et al. 48 suggested an interesting experimental test in the form of particle flows in three-jet $(q \bar{q} g)$ and radiative two-jet $(q \bar{q} \gamma)$ events by observing the negative contribution of the third antenna. This test was carried out by the MARK II collaboration at PEP at $\sqrt{s}=29 \mathrm{GeV}[183$. with the result that in the angular region between the quark and antiquark jets fewer charged tracks were observed in the two-jet events than in the radiative three-jet events. Their result is shown in Fig. 22 (lower two frames).

To end this review of the studies of jets at PETRA and PEP, we briefly discuss the angle ordered perturbation theory, as this approach has been used to develop a parton shower Monte Carlo [39]. In this approach, the phase space of soft gluon emission is restricted using an angle ordering criterion, which allows one to take into account the interference (colour coherence) approximately, and hence it reproduces the string and string-like effects discussed above. Both the LUND fragmentation model (PYTHIA in its modern incarnation) and the parton shower Monte Carlo models (such as HERWIG) describe the $e^{+} e^{-}$data adequately. However, the main drawback of these models is that they do not (easily) match with the fixed order perturbation theory in next-to-leading and higher orders. The main obstacle is that fixed order perturbation theory has soft and collinear singularities that give rise to logarithmic enhancement of higher order contributions. These enhanced terms should be summed to all orders. However, there is no unique way of doing this. For example, the $p_{T}$-ordered and the angular ordered showers can both be arranged to resum these logarithms. Matching with a fixed order perturbation theory is more easily achieved in $p_{T}$ ordered showers which, however, do not have the colour coherence needed by the low-energy $e^{+} e^{-}$data. It is the other way around with the angle-ordered showers. We will discuss these aspects further in the next section. 

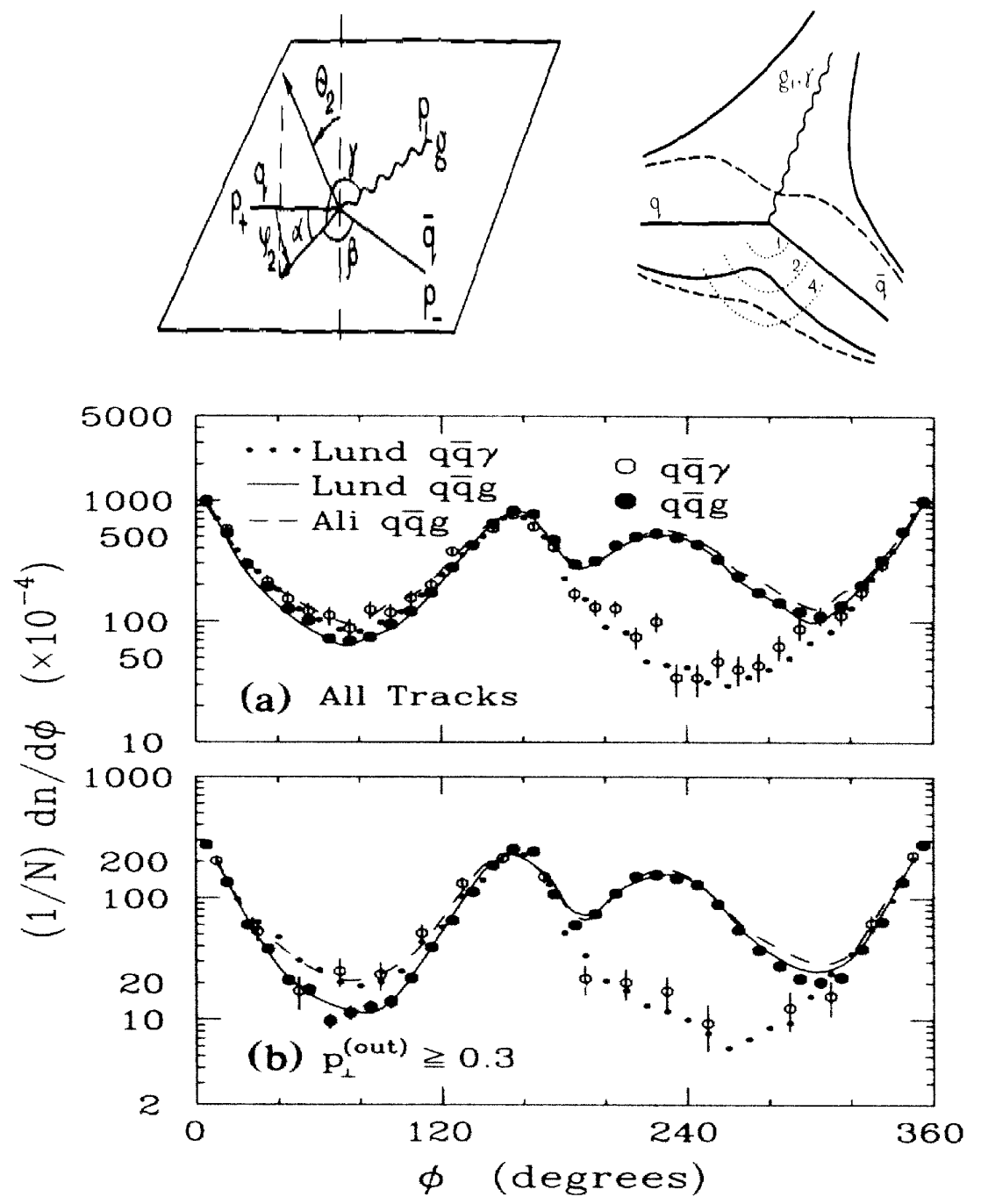

Fig. 22. Upper left-hand frame: Kinematics of non-jet radiation in three-jet events; Upper right-hand frame: Directivity diagram of the soft particle flows, projected on to the $q \bar{q} \gamma$ (dashed lines) and $q \bar{q} g$ (solid line) event planes. Dotted circles show the constant levels of density flow $[W(\phi)=1,2,4]$ (from Azimov et al. 48]). Lower frame: The charged-track density as a function of the event-plane angle $\phi$. The angular region between $\phi=0^{\circ}$ and $\phi=150^{\circ}$ separates the $q$ and $\bar{q}$ for the $q \bar{q} \gamma$ events and for $65 \%$ of the $q \bar{q} g$ events (from the MARK II collaboration 183 ).

\section{Jets in QCD and at LEP}

In this section we review the salient features of jets at LEP which were helpful in testing some of the basic elements of QCD more precisely. Just as in the preceding section, we recall the main detectors at LEP, ALEPH, DELPHI, OPAL, and L3, which collected large data samples (typically, 4 million hadronic events around the $Z$ resonance for each of the four LEP experiments. In the second stage, LEP2, the beam 

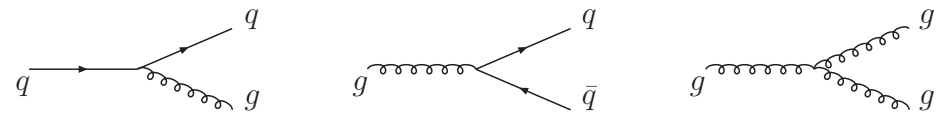

Fig. 23. The three basic splitting processes of quarks and gluons into pairs of quarks and gluons.

energy was increased to about $103 \mathrm{GeV}$. These detectors proved to be very powerful tools in carrying out precision electroweak and QCD physics.

\subsection{Quark/Gluon cascades}

Electric charges which are accelerated, reduce their energy by radiating photons preferentially collinear with the flight direction of the charge. This is a general feature of gauge theories and, specifically, collinear radiation is predicted in QCD processes in which quarks and gluons are produced with high energies. If the observed partons carry away a fraction $z$ of the parent partons, the splitting functions [184, $c f$. Fig. 23,

$$
\begin{aligned}
d P[q \rightarrow q+g(z)] & =\frac{\alpha_{s}}{2 \pi} C_{F} \frac{1+(1-z)^{2}}{z} d z \frac{d Q^{2}}{Q^{2}} \equiv \frac{\alpha_{s}}{2 \pi} P_{g q}(z) d z \frac{d Q^{2}}{Q^{2}}, \\
d P[q \rightarrow q(z)+g] & =\frac{\alpha_{s}}{2 \pi}\left[C_{F} \frac{1+z^{2}}{(1-z)_{+}}+2 \delta(1-z)\right] d z \frac{d Q^{2}}{Q^{2}} \equiv \frac{\alpha_{s}}{2 \pi} P_{q q}(z) d z \frac{d Q^{2}}{Q^{2}}, \\
d P[g \rightarrow q(z)+\bar{q}] & =\frac{\alpha_{s}}{2 \pi} T_{R}\left[z^{2}+(1-z)^{2}\right] d z \frac{d Q^{2}}{Q^{2}} \equiv \frac{\alpha_{s}}{2 \pi} P_{q g}(z) d z \frac{d Q^{2}}{Q^{2}}, \\
d P[g \rightarrow g+g(z)] & =\frac{\alpha_{s}}{2 \pi}\left(2 C_{A}\left[\frac{1-z}{z}+z(1-z)+\frac{z}{(1-z)_{+}}\right]+\left[\frac{11}{2}-\frac{n_{f}}{3}\right] \delta(1-z)\right) d z \frac{d Q^{2}}{Q^{2}} \\
& \equiv \frac{\alpha_{s}}{2 \pi} P_{g g}(z) d z \frac{d Q^{2}}{Q^{2}},
\end{aligned}
$$

universally predict collinear splittings, with $Q^{2} \simeq z(1-z) E^{2} \Theta^{2}$ denoting the invariant mass of the final parton pair. The notation $[F(z)]_{+}$defines a distribution such that for any sufficiently regular function $f(z)$,

$$
\int_{0}^{1} d z f(z)[F(z)]_{+}=\int_{0}^{1} d z(f(z)-f(1)) F(z) .
$$

The bremsstrahlung splittings $q \rightarrow q g$ and $g \rightarrow g g$ preferentially generate soft radiation spectra in the limit $z \rightarrow 0$. The group characteristics are $C_{F}=4 / 3, C_{A}=3$ and $T_{R}=1 / 2$ for $\mathrm{SU}(3)_{C}$ of $\mathrm{QCD}$.

Repeated splittings generate cascades of collimated quarks and gluons. Since the lifetime of the final-state pair in the splitting processes is long, $\tau^{*} \sim E / Q^{2}$, the cascade is expected to be described by a sequence of probabilities and not by interfering quantum-mechanical amplitudes. If the branching occurs at a value $Q^{2}$ without any radiation between the initial maximum value $Q_{\max }^{2}$ and $Q^{2}$, the probability is given by

$$
d \mathcal{P}_{a \rightarrow b c}=\frac{\alpha_{s}}{2 \pi} P_{a \rightarrow b c}(z) d z \frac{d Q^{2}}{Q^{2}} \exp \left[-\sum_{b^{\prime}, c^{\prime}} \int_{Q^{2}}^{Q_{\max }^{2}} \frac{d Q^{\prime 2}}{Q^{\prime 2}} \int d z^{\prime} \frac{\alpha_{s}}{2 \pi} \hat{P}_{a \rightarrow b^{\prime} c^{\prime}}\left(z^{\prime}\right)\right],
$$


where the exponential Sudakov factor [71] accounts for the non-radiation probability. Here $\hat{P}_{a \rightarrow b^{\prime} c^{\prime}}\left(z^{\prime}\right)$ are the same functions as $P_{a \rightarrow b^{\prime} c^{\prime}}\left(z^{\prime}\right)$, defined in Eq. (51) except for the regularization terms at $z^{\prime} \rightarrow 1$.

However, the branching probability Eq. (53) is refined by an important coherence effect. If in electrodynamics a photon splits into an electron-positron pair, the pair can emit photons only at angles less than the angle between the charged pair as photons propagating at larger angles would see coherent electron+positron states which, being neutral, cannot radiate. As a result, the emission angles are ordered in the sequence $\Theta_{1}>\Theta_{2}>\ldots$ This effect is also predicted in QCD 39]. The only difference arises from the fact that the coherent superposition adds up the colour charges of the daughter partons to the non-zero value of the parent colour charge so that wide-angle splitting is generated at a non-zero rate. In addition to the angular ordering, non-resolved infrared radiation restricts the energy fractions of the partons in the cascades. These restrictions on energies and angles can be mapped into the boundary values of the Sudakov integral after re-expressing the invariant mass by the angle between the momenta of the daughter partons.

The cascading of the primordial quarks and gluons affects the observed hadron distributions within the jets. In particular, energy spectra are softened through the cascading mechanism, multiplicities increase strongly with energy, and quark and gluon jets will develop different profiles. Formulated for simplicity by neglecting the change in $k_{T}$, and restricting to one parton species, the energy dependence of the fragmentation function is described by the DGLAP equation [184 185 186 187]:

$$
\frac{\partial D\left(z, Q^{2}\right)}{\partial \log Q^{2} / \Lambda^{2}}=\frac{\alpha_{s}\left(Q^{2}\right)}{2 \pi} \int_{z}^{1} \frac{d \zeta}{\zeta} P(\zeta) D\left(\frac{z}{\zeta}, Q^{2}\right) .
$$

The splitting function $P(\zeta)$ consists of two parts (see, Eq. (51). The first part $P$ describes the standard component and accounts for the accumulation of particles at $z$ generated by the splitting of partons at $\zeta \geq z$, the second part accounts for the loss of particles at $z$ due to splitting to smaller energy values. In the parton model, the function $D$ depends only on the variable $\frac{z}{\zeta}$ but not on $Q^{2}$. This would yield an scale-invariant fragmentation function $D\left(\frac{z}{\zeta}\right)$. In QCD, this is obviously modified. The solution of the above equation leads to striking effects which modify the predictions of the scale-invariant parton model. In particular:

(i) For large $z$ values beyond 0.2 the spectrum decreases with increasing energy while the particles accumulate at small $z$. The loss of particles by splitting at large $z$ is bigger than the gain by splitting from yet higher $\zeta$ values. This is naturally opposite at small $z$ values.

(ii) The constant plateau (characterized by $D(z)$ as in Eq. (5)) in the parton model generates a multiplicity of particles which increases logarithmically with the length of the plateau $\sim \log \left(\sqrt{s} / m_{h}\right)$. Multiple splittings raise the multiplicity much more strongly. Solving Eq. (54) for the multiplicity, given by the integrated fragmentation function, predicts a rise with energy stronger than exponential:

$$
n(s) \sim \exp \left(\alpha_{s}^{-1 / 2}(s)\right) \sim \exp \left(\log ^{1 / 2} s / \Lambda^{2}\right) .
$$

In addition, the flat plateau in $\log z^{-1}$ is deformed to a humpback of Gaussian character [188, with centre $\left[\log z^{-1}\right]_{\max } \sim \log s / \Lambda^{2}$ and width $\sigma \sim \log ^{3 / 4} s / \Lambda^{2}$. Experimental proof for the energy dependence of the fragmentation function $D\left(z, Q^{2}\right)$ and the formation of the humpback at small $z$ is presented in Fig 24.

(iii) While the first splitting of a quark jet $q \rightarrow q g$ is determined by $C_{F}=4 / 3$, the first splitting of a gluon jet $g \rightarrow g g$ is determined by the bigger Casimir invariant $C_{A}=3$. Thus, the large average colour of gluons compared with quarks should 

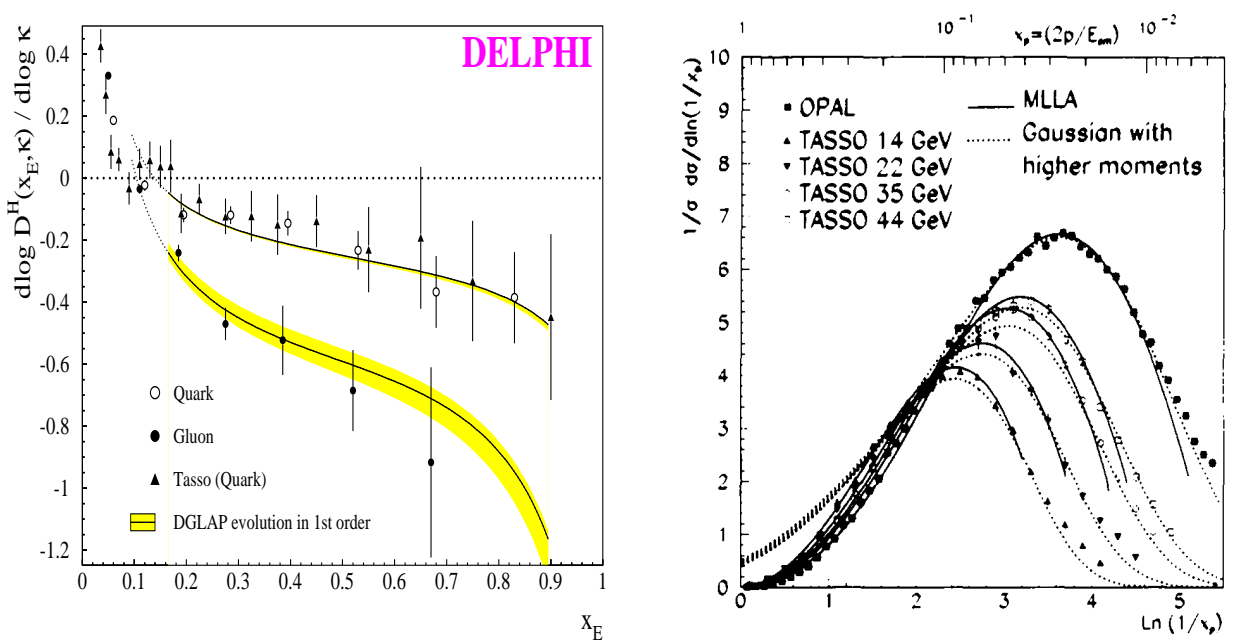

Fig. 24. (a) Scaling violations in quark and gluon jets (using the DURHAM algorithm). The solid curves show the DGLAP evolution and the dotted parts of the curves are extrapolation outside the fit range [DELPHI [189]]. (b) Measurements of the $\ln \left(1 / x_{p}\right)$ distributions for the center-of-mass energies between 14 and $91 \mathrm{GeV}$ and comparison with the MLLA 188 and a Gaussian 190. This figure is referred as the humpback plateau of the gluon fragmentation function for small $x$ values in the text [OPAL [191]].

increase the multiplicity of gluon jets with regard to quark jets asymptotically in the ratio $C_{A} / C_{F}=9 / 4$. Similarly, since $d N_{g / q} \sim C_{A / F} d \log \Theta$, the angular widths of quark and gluon jets are different, $\Theta_{g}=\Theta_{q}^{C_{F} / C_{A}}$, viz. gluon jets are wider than quark jets. Even though the asymptotic limit has not been reached yet, the particle multiplicities in gluon jets have been shown significantly larger than in quark jets [192: $n_{g} / n_{q}>3 / 2$. For a recent discussion of particle multiplicities, we refer to [193].

(iv) Small-angle gluon radiation off heavy quarks $Q=c, b$ is suppressed compared to light quarks [194, and the logarithmic enhancement of the particle yield is restricted to infrared gluon configurations.

\subsection{Multijets at LEP}

Increasing the energy from the PETRA regime of about $\sqrt{s}=46 \mathrm{GeV}$ to the LEP regime by factors of two and five in the two phases of LEP, $Z$-boson runs with $\sqrt{s}=$ $91 \mathrm{GeV}$ and beyond with $\sqrt{s}$ up to $206 \mathrm{GeV}$, provided two opportunities: the experimental analysis of multijet final states [34] and the study of the jet properties over a large range in energy [195. This allows a more precise measurement of two fundamental characteristics of QCD [196, the running of the QCD coupling with energy as predicted by asymptotic freedom, and the three-gluon coupling, a fundamental ingredient of asymptotic freedom. We discuss these measurements below in turn.

\subsection{Inclusive jet observables and determination of $\alpha_{s}\left(M_{Z}\right)$ at LEP}

All four experiments at LEP, DELPHI [197], OPAL [198, L3 [199], and ALEPH [200], undertook measurements of the inclusive jet (or event shape) variables and their moments. In these analyses, the next-to-leading order $\left(O\left(\alpha_{s}^{2}\right)\right)$ perturbative QCD 
calculations for the inclusive observables and event shape distributions, discussed in the context of three-jet events at PETRA, were augmented by theoretical estimates in the next-to-leading-log approximation (NLLA) 201202 and others in which the $O\left(\alpha_{s}^{2}\right)$ and NLLA schemes were combined 203. It is helpful to explain the NLLA in more detail. For a generic shape variable, $y$, well-defined in pertrubation theory (i.e., infrared and collinear safe), the typical leading behaviour at small $y$ is

$$
\frac{1}{\sigma} \frac{d \sigma_{n}}{d y} \sim \alpha_{s}^{n} \frac{1}{y} \ln ^{2 n-1} \frac{1}{y} .
$$

The normalized event shape cross section $R(y)$ defined as

$$
R(y) \equiv \int_{0}^{y} d y \frac{1}{\sigma} \frac{d \sigma}{d y}
$$

then has the expansion

$$
R_{n}(y) \sim \alpha_{s}^{n} \ln ^{2 n}(1 / y) \equiv \alpha_{s} L^{2 n}
$$

Whenever $L$ is large, one can improve the range and accuracy of perturbative predictions by identifying these logarithmically-enhanced terms and resum them to all orders. For a class of variables for which the leading logarithmic contributions exponentiate, i.e., variables which in the small-y range yield the logarithm of the shape cross-section in the form $\ln R(y) \sim L g_{1}\left(\alpha_{s} L\right)$, where $g_{1}\left(\alpha_{s} L\right)$ has a power series expansion in $\alpha_{s} L$, one has 203]

$$
R(y)=C\left(\alpha_{s}\right) \Sigma\left(y, \alpha_{s}\right)+D\left(y, \alpha_{s}\right),
$$

with $C\left(\alpha_{s}\right)=1+\sum_{n=1}^{\infty} C_{n}\left(\alpha_{s} / 2 \pi\right)^{n}$ and $\ln \Sigma\left(y, \alpha_{s}\right)=L g_{1}\left(\alpha_{s} L\right)+g_{2}\left(\alpha_{s} L\right)+\alpha_{s} g_{3}\left(\alpha_{s} L\right)+$ $\ldots$. The function $g_{1}\left(\alpha_{s} L\right)$ resums all the leading contributions of the form $\alpha_{s} L^{n+1}$ (defining the LAA approximation) and $g_{2}\left(\alpha_{s} L\right)$ contains the next-to-leading logarithmic corrections $\alpha_{s}^{n} L^{n}$ (defining the NLLA) and so on.

For the hadronisation effects, either the Monte Carlo based hadronisation models, PYTHIA 4950, HERWIG [51] and ARIADNE 204] were used, or, alternatively, non-perturbative power-correction formulae derived in [205 206 207] were employed. This latter ansatz provides an additive term to the perturbative QCD estimate in mean event shape variables. Studying the energy-dependence of these mean variables $\langle f\rangle$, defined as

$$
\langle f\rangle=\frac{1}{\sigma_{\text {tot }}} \int f \frac{d f}{d \sigma}=\left\langle f_{\text {pert }}\right\rangle+\left\langle f_{\text {pow }}\left(\alpha_{0}\right)\right\rangle,
$$

yielded a measurement of $\alpha_{s}(\mu)$ and $\alpha_{0}$, the non-perturbative parameter characterising the power corrections. We have discussed the $O\left(\alpha_{s}^{2}\right)$ calculations of $f_{\text {pert }}$ for several observables (thrust, the Fox-Wolfram shape variable $C$, etc.) in section 5.4. Explicit formulae for $f_{\text {pow }}$ are given by Dokshitzer et al. [205 206 207], and can also be seen, for example, in the DELPHI analysis [197] of the LEP2 data. For related discussion of event shapes in $e^{+} e^{-}$annihilation and deep inelastic scattering, we refer to [208. A typical measurement along these lines is shown in Fig. 25], in which the measured mean values of $\langle 1-T\rangle$, and the scaled heavy jet mass $\left\langle M_{h}^{2} / E_{\text {vis }}^{2}\right\rangle$ are shown as a function of the center-of-mass energy together with the results of the fits. The dotted curves in these figures show the perturbative QCD part only. It is obvious from this figure that even at the highest LEP2 energy, non-perturbative power corrections are not small. The fits yield $\alpha_{s}\left(M_{Z}\right)=0.1191 \pm 0.0015 \pm 0.0051$ from $\langle 1-T\rangle$ and a very consistent value from the other observable $\left\langle M_{h}^{2} / E_{\mathrm{vis}}^{2}\right\rangle$. However, the value of $\alpha_{0}(2 \mathrm{GeV})$, the measure of power corrections, differs by about $20 \%$ from the two measurements, 


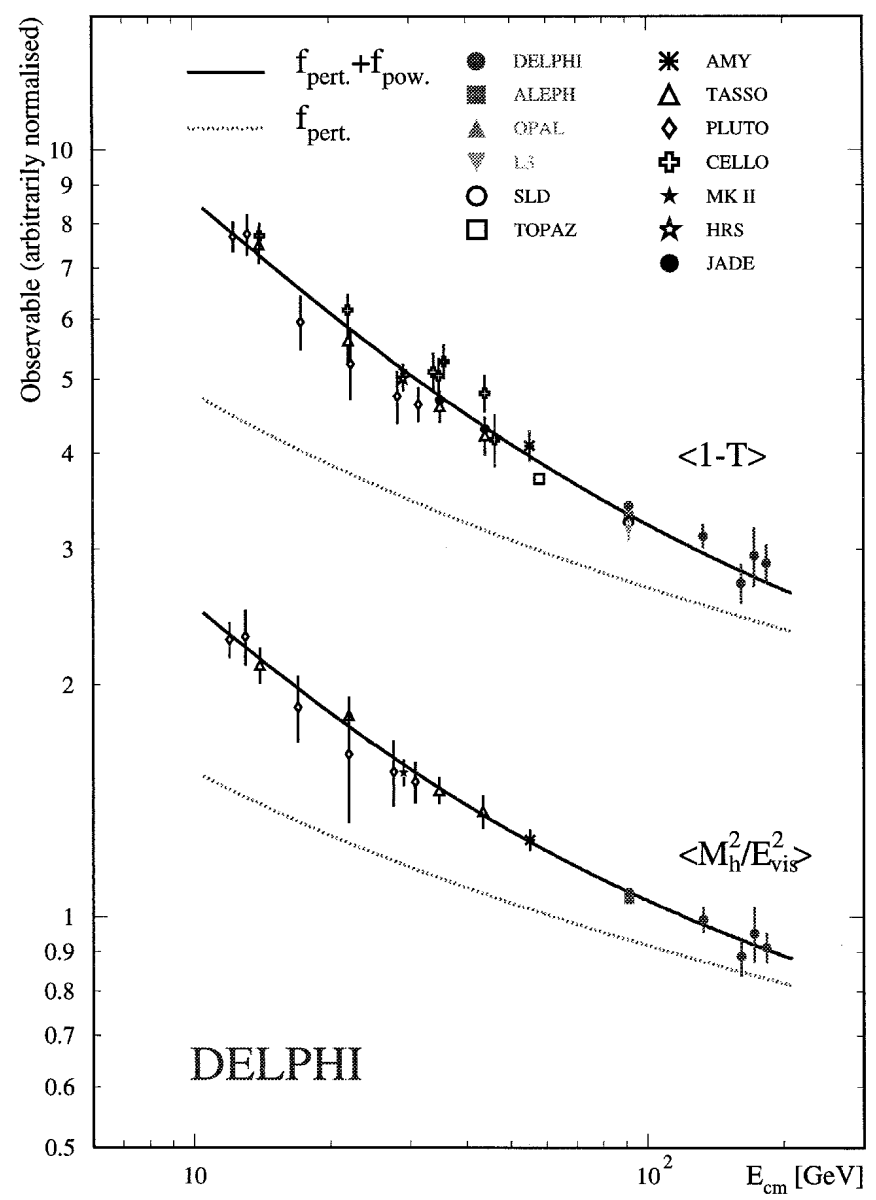

Fig. 25. Measured mean values of the observables $\langle 1-T\rangle$ and $\left\langle M_{h}^{2} / E_{\mathrm{vis}}^{2}\right\rangle$ as a function of the center-of-mass energy. The solid lines present the results of the fits including power corrections and the dotted lines show the perturbative part only. (From DELPHI [197]).

showing considerable non-universality in the parametrisation of $f_{\text {pow }}$ by Dokshitzer et al. 205 206 207. Along the same lines, the L3 collaboration measured the mean values of several global shape parameters. For all these variables, the same theoretical frameworks 201202203] as discussed above in the context of the DELPHI measurements were used. To compare these calculations at parton level with the experimental measurements, the effects of hadronisation and decays were corrected for using the JETSET PS (parton shower) Monte Carlo program. We display in Fig. 26 the measured distributions in thrust and the variable $C$ at $\sqrt{s}=206.2 \mathrm{GeV}$ and comparison with the QCD fits, showing excellent agreement. To determine $\alpha_{s}$ at each energy, the formalism in [203] is used, which yielded $\alpha_{s}\left(M_{Z}\right)=0.1227 \pm 0.0012 \pm 0.0058$ [199].

\subsubsection{Jet rates}

Due to the high energy at LEP1, up to four jets could be resolved experimentally. The number of resolved jets depends strongly on the criterion by which the jets are defined. Early definitions had used the JADE recombination scheme which combined 

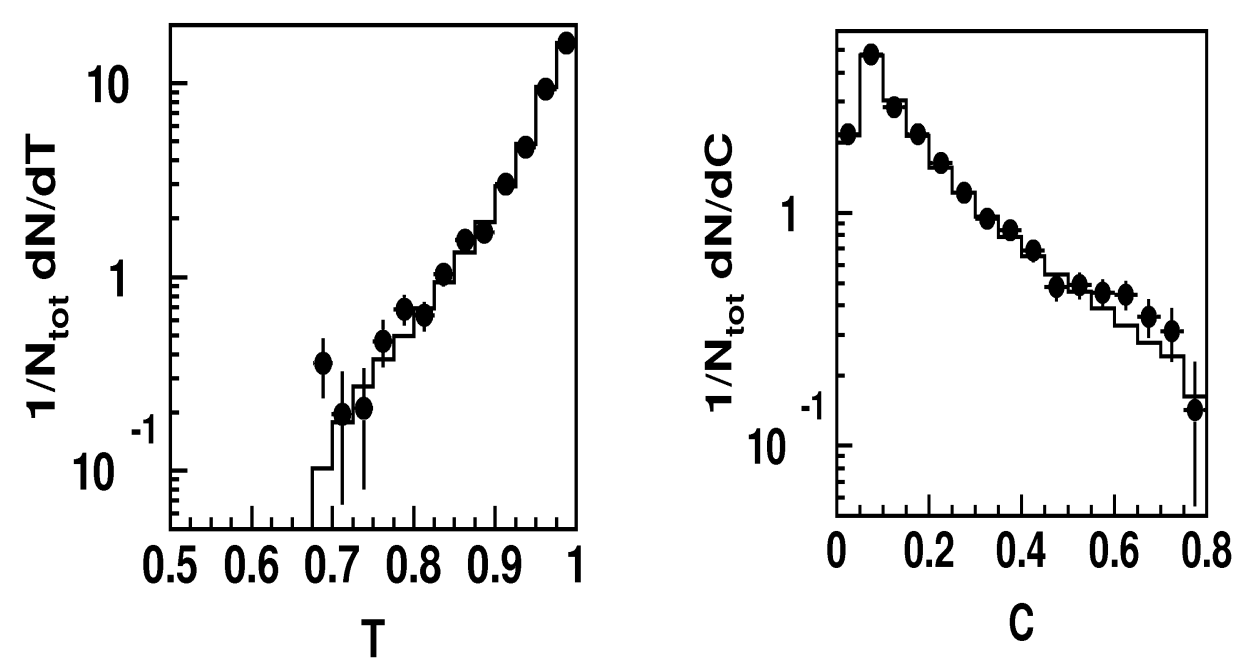

Fig. 26. Measured distributions of thrust, T, (left-hand frame) and the $C$-parameter in comparison with QCD predictions at $\sqrt{s}=206.2 \mathrm{GeV}$ [From L3 [199]].

particle pairs on the experimental side, and equally quark/gluon parton pairs on the theoretical side [166], for scaled invariant masses $M_{i j}^{2} / s=y$ below a cut-off value $\leq y_{\text {cut }}$. In the DURHAM scheme [78] the invariant mass was replaced by $M_{i j}^{2}=$ $2 \min \left(E_{i}^{2}, E_{j}^{2}\right)\left[1-\cos \theta_{i j}\right]$, essentially the transverse momentum between the particles or partons for small angles. The cut-off value $y_{\text {cut }}$ was chosen typically from $10^{-1}$ down to $10^{-3}$. Small values of $y_{\text {cut }}$ naturally lead to large numbers of jets while the number of jets is reduced if $y_{\text {cut }}$ is increased. The cross section for 3 -jet events,

$$
\sigma_{3}[y]=\left(\frac{\alpha_{s}}{\pi}\right) \sigma_{31}+\left(\frac{\alpha_{s}}{\pi}\right)^{2} \sigma_{32}+\left(\frac{\alpha_{s}}{\pi}\right)^{3} \sigma_{33}
$$

has been calculated up to third order in the QCD coupling 209]. NLO corrections to the four-jet rates in the process $e^{+} e^{-} \rightarrow \gamma^{*}, Z \rightarrow 4$ jets were done around 1996 by Dixon and Signer 210211] and subsequently by Nagy and Trocsanyi 212. The experimental number of jets in $Z$ decays is displayed in Fig. 27 and compared with a parton shower Monte Carlo prediction (Jetset partons), and including hadronisation effects (Jetset hadrons). Evidently, for $y_{\text {cut }}$ below $10^{-2}$ up to four jets can clearly be identified at LEP1 [213. A dedicated effort to test QCD and determine $\alpha_{s}$ was undertaken by the combined JADE (at PETRA) and OPAL (at LEP) collaborations 198, giving a considerably larger lever arm in energy from $35 \mathrm{GeV}$ to 189 $\mathrm{GeV}$. The observables used in this (JADE + OPAL) analysis are exclusively based on the multiplicities of hadronic jets. The $n$-jet fractions, $R_{n}$ were defined using the JADE 44, Durham 78, and Aachen/Cambridge 81 algorithms. We show in Fig. 28 the three-jet fraction $R_{3}$ obtained with the JADE and Durham jet algorithms versus the center-of-mass energy. (The result from the Aachen/Cambridge algorithm can be seen in [198.) Here, the data from PETRA and LEP are compared with the $O\left(\alpha_{s}^{2}\right)$ prediction. The renormalisation scale dependence is shown by the scale parameter $x_{\mu}=\mu / \sqrt{s}$, with the solid lines corresponding to a fixed value $x_{\mu}=1$, and the dashed lines are the results obtained with a fitted scale, indicated on the figure. This and related analyses reported in [198 yield a rather precise value for the QCD coupling constant $\alpha_{s}\left(M_{Z}\right)=0.1187_{-0.0019}^{0.0034}$. At LEP2 (up to $\left.\sqrt{s}=206 \mathrm{GeV}\right)$, the highest 


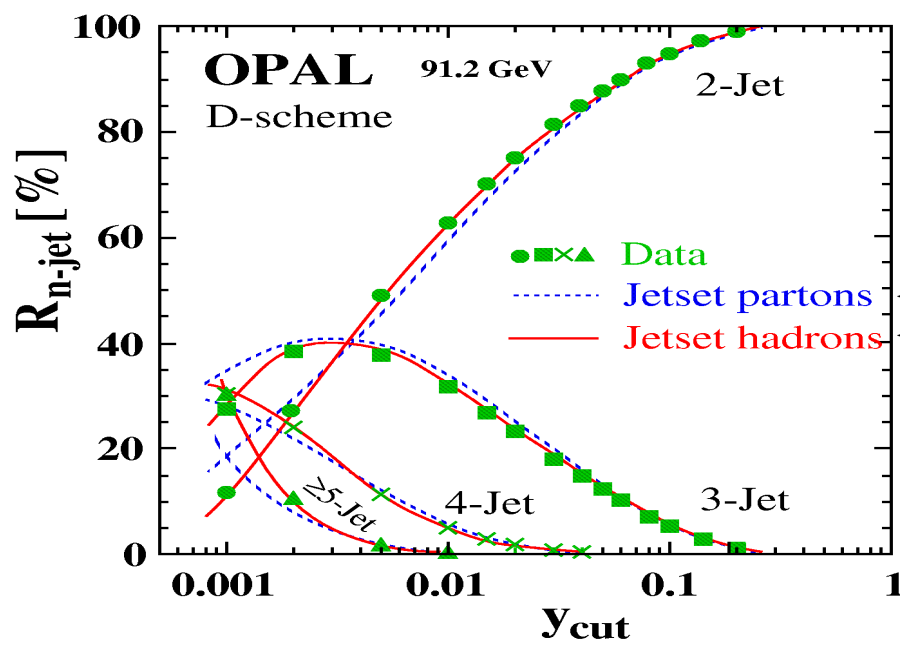

Fig. 27. Relative production rates of $n$-jet events defined in the Durham jet algorithm scheme 78 as a function of the jet resolution parameter $y_{\text {cut }}$. The data are compared to model calculations before and after the hadronisation process as indicated on the figure [OPAL 213]].
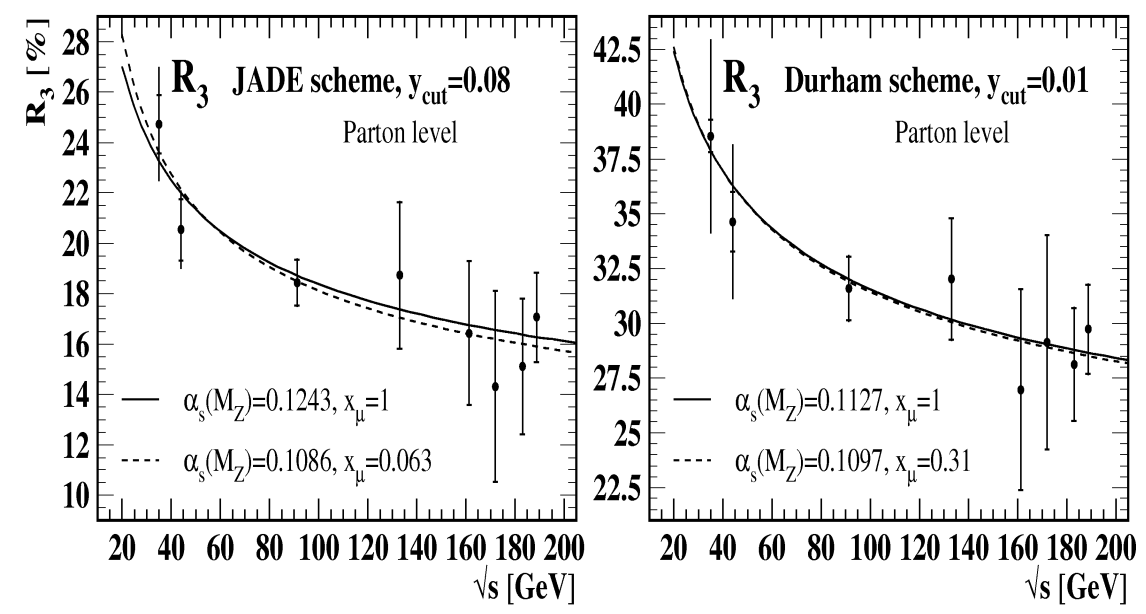

Fig. 28. Measured 3-jet fraction as obtained with the JADE (left-hand frame) and Durham schemes (right-hand frame) at parton level versus the c.m.s. energy $\sqrt{s}$. The data shown are from the JADE and OPAL collaboration, and the curves are the $O\left(\alpha_{s}^{2}\right)$ predictions at a fixed scale (solid lines) and for the fitted values of the scale (dashed lines). [From OPAL [198].]

jet multiplicity measured is five, obtained using the variable $y_{\text {cut }}$, and inclusive measurements are available for up to six jets. To match this data, NLO QCD corrections to five-jet production at LEP have been carried out by Frederix et al. [214, and the fixed-order perturbative results have been compared with the LEP1 data from ALEPH [200. Two observables have been used for this comparison:

(i) Differential distribution with respect to the five-jet resolution parameter $y_{45}$, the maximum value of $y_{\text {cut }}$ such that a given event is classified as a five-jet event by the 
DURHAM jet algorithm [78]:

$$
\int_{y_{\text {cut }}}^{1} d y_{45} \frac{d \sigma}{d y_{45}}=\sigma_{\text {incl }}^{5-\text { jet }}\left(y_{\text {cut }}\right),
$$

where $\sigma_{\text {incl }}^{5-\text { jet }}$ is the inclusive five-jet production cross section in $e^{+} e^{-}$annihilation. (ii) Five-jet rate $R_{5}\left(y_{\text {cut }}\right)$, defined as follows:

$$
R_{5}\left(y_{\text {cut }}\right)=\frac{\sigma_{\text {excl }}^{5-\text { jet }}\left(y_{\text {cut }}\right)}{\sigma_{\text {tot }}}
$$

where $\sigma_{\text {excl }}^{5-\text { jet }}\left(y_{\text {cut }}\right)$ is the exclusive five-jet production cross section. This is also calculated using the Durham jet algorithm by requiring that exactly five jets are reconstructed. Both observables, $\sigma_{\text {tot }}^{-1} d \sigma / d \ln y_{45}^{-1}$ and $R_{5}\left(y_{\text {cut }}\right)$, can be written as a series in $\alpha_{s}(\mu)$, with the leading contributions starting in $O\left(\alpha_{s}^{3}\right)$. A comparison of the leading order and next-to leading order predictions for $(1 / \sigma) d \sigma / d \ln y_{45}$ vs. $\ln \left(y_{45}\right)$ and the exclusive 5 -jet fraction $R_{5}$ vs. $\ln \left(y_{\text {cut }}\right)$ is shown in Fig. 29 with the ALEPH data in a limited range of these variables (perturbative regime). Hadronisation effects have been estimated using the SHERPA MC [52. As is typical of NLO calculations, scale dependence is significantly reduced compared to the LO calculations. Agreement between data and NLO theory is impressive and has been used to extract a value of $\alpha_{s}$, obtaining $\alpha_{s}\left(M_{Z}\right)=0.1156_{-0.0034}^{+0.0041}$, which is in excellent agreement with the worldaverage discussed below. The limitation of using fixed-order perturbative QCD in describing the $e^{+} e^{-}$data can be seen in the ALEPH data shown in Fig. 30, which show a characteristic turnover shape around $-\ln y_{45} \simeq 7.5$. In this region, perturbation theory fails and a resummation (equivalently showers) have to be included to describe the data. This underscores the importance of having MC generators which include showers.

In Fig. [30, the ALEPH LEP1 data for $\sigma_{\text {tot }}^{-1} d \sigma / d \ln y_{45}^{-1}$ are compared with the hadron level predictions of three event generators (the numbers denote the various versions of these Monte Carlo programmes), PYTHIA6.1 215, HERWIG6.1 [216] and ARIADNE4.1 204. Agreement between data 200] and these MCs is generally good. However, as shown in the upper frame of fig. 30, hadronic corrections are large, varying from 0.5 to 1.5 in this range. In addition, differences between hadronisation corrections among the MCs are as large as $25 \%$. This deficiency can be overcome to some extent by matching the parton shower and high multiplicity matrix elements, as, for example, proposed in 217. This matching procedure has been implemented in the SHERPA event generator [52] and results in improved agreement between the $\mathrm{MC}$ and fixed-order perturbative description.

\subsubsection{The gluon self-coupling}

The study of the three-gluon coupling in gluon splitting to two gluons requires four (or more) jets in $e^{+} e^{-}$annihilation. A variety of angular correlations and energy distributions, see [218-221, can be exploited to signal the three-gluon coupling of QCD.

The sensitivity to angular distributions may be illustrated in a transparent example [218. A virtual gluon, radiated off the quarks in the process $e^{+} e^{-} \rightarrow q \bar{q} g^{*}$, is polarised preferentially in the production plane. The subsequent splitting of the virtual gluon into two real gluons or a quark-antiquark pair is sensitive to the azimuthal 

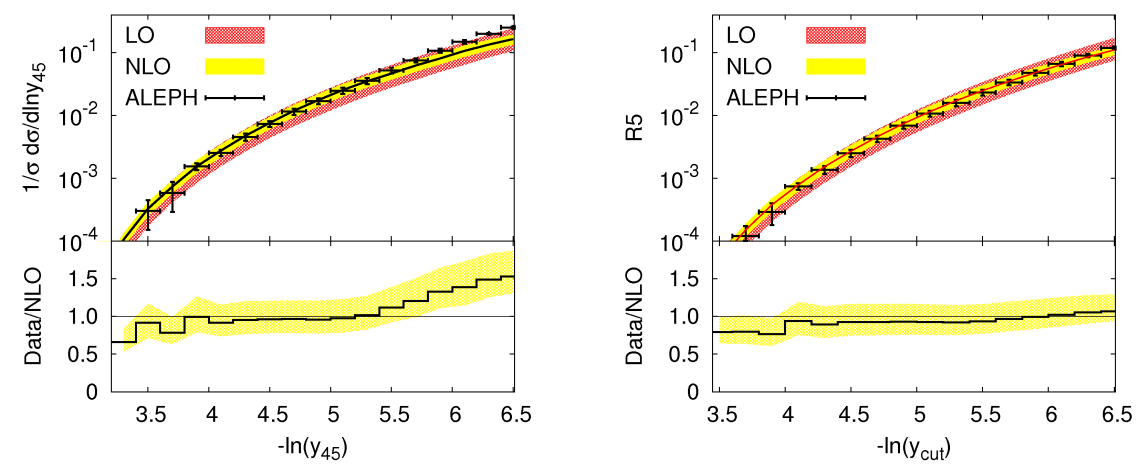

Fig. 29. ALEPH LEP1 data 200] compared to leading and next-to-leading order predictions for $1 / \sigma d \sigma / d \ln y_{45}$ plotted against $\ln y_{45}$ (left-hand frame) and $R-5$ plotted against $\ln \left(y_{\text {cut }}\right)$ (right-hand frame) without hadronisation corrections. The uncertainty bands are obtained by varying the scale in the interval $0.15 M_{Z}<\mu<0.6 M_{Z}$, and the solid lines refer to NLO QCD evaluated at $\alpha_{s}\left(M_{Z}\right)=0.118$ and $\mu=0.3 M_{Z}$. [From Ref. [214]].

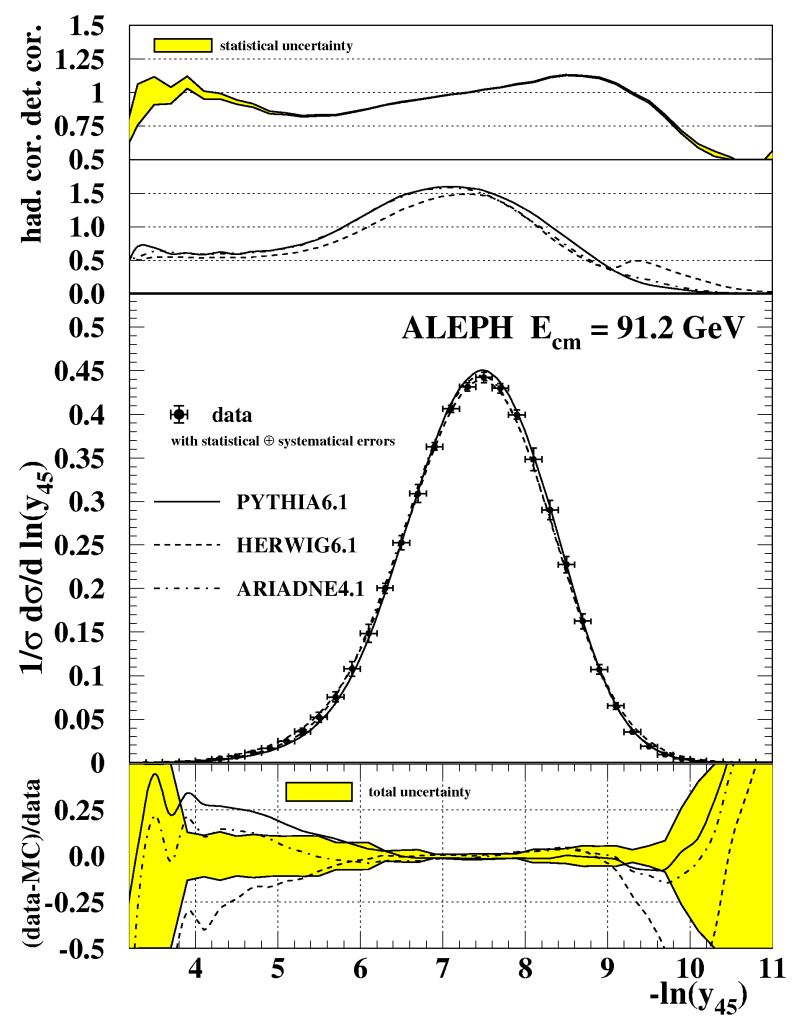

Fig. 30. ALEPH data 200 for the $y_{45}$ distribution at LEP1 compared to PYTHIA,HERWIG and ARIADNE Monte Carlo results. The upper frames show detector and hadronisation corrections, respectively. The lowest frame shows the relative difference between data and event generator predictions. [Figure attributed to H. Stenzel in Ref. [214]]. 
angle $\phi$ between the $g^{*}$ polarisation vector and the decay planes:

$$
\begin{aligned}
& n_{g g}=\frac{\left[1-z+z^{2}\right]^{2}}{z(1-z)}+z(1-z) \cos 2 \phi, \\
& n_{q \bar{q}}=\frac{1}{2}\left[z^{2}+(1-z)^{2}\right]-z(1-z) \cos 2 \phi .
\end{aligned}
$$

As a result, the polarisation vector and the decay plane tend to be aligned in gluon splitting to two gluons. In contrast, if the gluon splits to a quark-antiquark pair, the decay plane tends to orient itself perpendicular to the polarisation vector.

The azimuthal distribution can be studied experimentally by measuring the angle between the planes spanned by the two hardest and the two softest jets. In an abelian theory the $\phi$ asymmetry is large and the two planes would orient themselves perpendicular to each other. By contrast, since the $\phi$-independent term in gluon splitting to two gluons in QCD is large, the azimuthal asymmetry in this process is predicted to be small, but the two planes should have a tendency to orient themselves parallel rather than perpendicular. This is borne out by experimental analyses 222 indeed, as demonstrated in Fig. 31

Quite generally, four jets are produced in $e^{+} e^{-}$annihilation by three mechanisms: double gluon bremsstrahlung, gluon splitting to two gluons, and gluon splitting to a quark-antiquark pair. The cross section [140141] can be decomposed accordingly:

$$
\sigma_{4}=\left(\frac{\alpha_{s}}{\pi}\right)^{2} C_{F}\left[C_{F} \sigma_{b b}+C_{A} \sigma_{g g}+n_{f} T_{R} \sigma_{q q}\right]
$$

The first term accounts for double gluon bremsstrahlung $q \rightarrow q g$ and $\bar{q} \rightarrow \bar{q} g$, the second for gluon splitting to two gluons $g \rightarrow g g$, the third for gluon splitting to $n_{f}$ quark pairs $g \rightarrow q \bar{q}$. The Casimir group characteristics of the splitting vertices are $\left[C_{F}, C_{A}, T_{R}\right]=[4 / 3,3,1 / 2]$ in $\mathrm{QCD}$, while the corresponding characteristics are $[1,0,3]$ in an abelian theory. Measurements of their ratios yield [223]

$$
\begin{aligned}
& C_{A} / C_{F}=2.29 \pm 0.06[\text { stat. }] \pm 0.14[\text { syst. }], \\
& T_{R} / C_{F}=0.38 \pm 0.03[\text { stat. }] \pm 0.06[\text { syst. }],
\end{aligned}
$$

compared with the theoretical predictions $C_{A} / C_{F}=9 / 4$ and $T_{R} / C_{F}=3 / 8$ in QCD. Again we observe a strong signal of the three-gluon coupling in $C_{A}$, far away from zero in the abelian theory.

\subsubsection{QCD coupling and asymptotic freedom}

A large range of energies can be covered in the measurement of the QCD coupling $\alpha_{s}\left(Q^{2}\right)$, extending from $29 \mathrm{GeV}$ (at PEP) to $46 \mathrm{GeV}$ (at PETRA) up to about 206 $\mathrm{GeV}$ at LEP; the lever arm can be elongated down to $1.8 \mathrm{GeV}$ by including $\tau$ decays.

The QCD prediction for the running coupling $\alpha_{s}\left(Q^{2}\right)$ has been determined up to the 5th power [224225]. Keeping terms up to 2 nd order leads to the following expression

$$
\alpha_{s}\left(Q^{2}\right)=\frac{1}{\beta_{0} \log \left(Q^{2} / \Lambda^{2}\right)}-\frac{\beta_{1} \log \log \left(Q^{2} / \Lambda^{2}\right)}{\beta_{0}^{3} \log ^{2}\left(Q^{2} / \Lambda^{2}\right)}+\ldots,
$$

with $\beta_{0}=\left(33-2 n_{f}\right) / 12 \pi, \beta_{1}=\left(153-19 n_{f}\right) / 24 \pi^{2}, \ldots$ and $\Lambda \approx 200 \mathrm{MeV}$ denoting the QCD scale at which the coupling grows indefinitely. An ensemble of observables has been calculated in perturbative expansions in next-to-leading order NLO up to $\mathrm{N}^{3} \mathrm{LO}$. Most accurate are the totally inclusive observables, like total cross sections, 


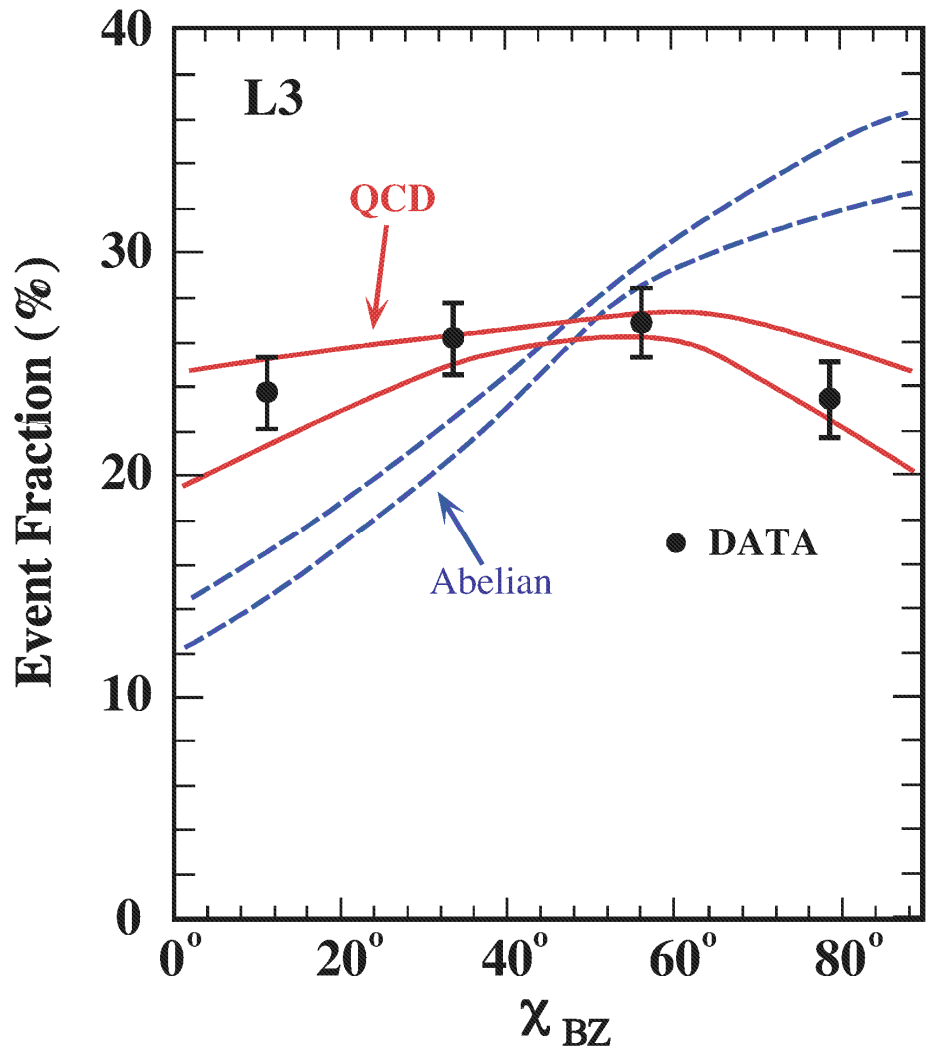

Fig. 31. The distribution of the azimuthal angle $\chi_{\mathrm{Bz}}$ (called $\phi$ in Eq. (59)) between the planes formed by the two most energetic jets and the two least energetic jets 222; the predictions of QCD including the gluon self-coupling are compared with an abelian theory without self-coupling of the gauge fields.

followed by jet cross sections and hadronic shape variables, like thrust. The estimates still depend significantly on the models used for calculating the shape variables in the non-perturbative region, see [226 227], for instance.

Combining the experimental measurements with the theoretical apparatus, the knowledge of the QCD coupling and its evolution with the energy is summarised in Fig. 32. The lever arm extends from the hadronic decays of the $\tau$ lepton throughout the PETRA range up to the highest energy values in the second phase of LEP. Including deep-inelastic lepton-nucleon scattering and jet production in hadron collisions, all the analyses are in remarkable agreement with the theoretical expectation from asymptotic freedom [3]. It has become customary to quote the value of $\alpha_{s}(\mu)$ measured in experiments by scaling the result to the scale $\mu=M_{Z}$ using the $\mathrm{RG}$ equation. This yields the current world average [228]

$$
\alpha_{s}\left(M_{Z}\right)=0.1184 \pm 0.0007 \text {. }
$$

This ends our discussion of jets in $e^{+} e^{-}$annihilation experiments and in QCD. In summary, essential parts of QCD jets can now be controlled at the level of typically ten percent $\left(\alpha_{s}\left(M_{Z}\right)\right.$ is known better than $\left.1 \%\right)$. Notabene the basic interactions and the strength of the quark-gluon coupling are proven to be asymptotically free. The high level of accuracy achieved in measuring the gauge couplings - weak, electromagnetic 

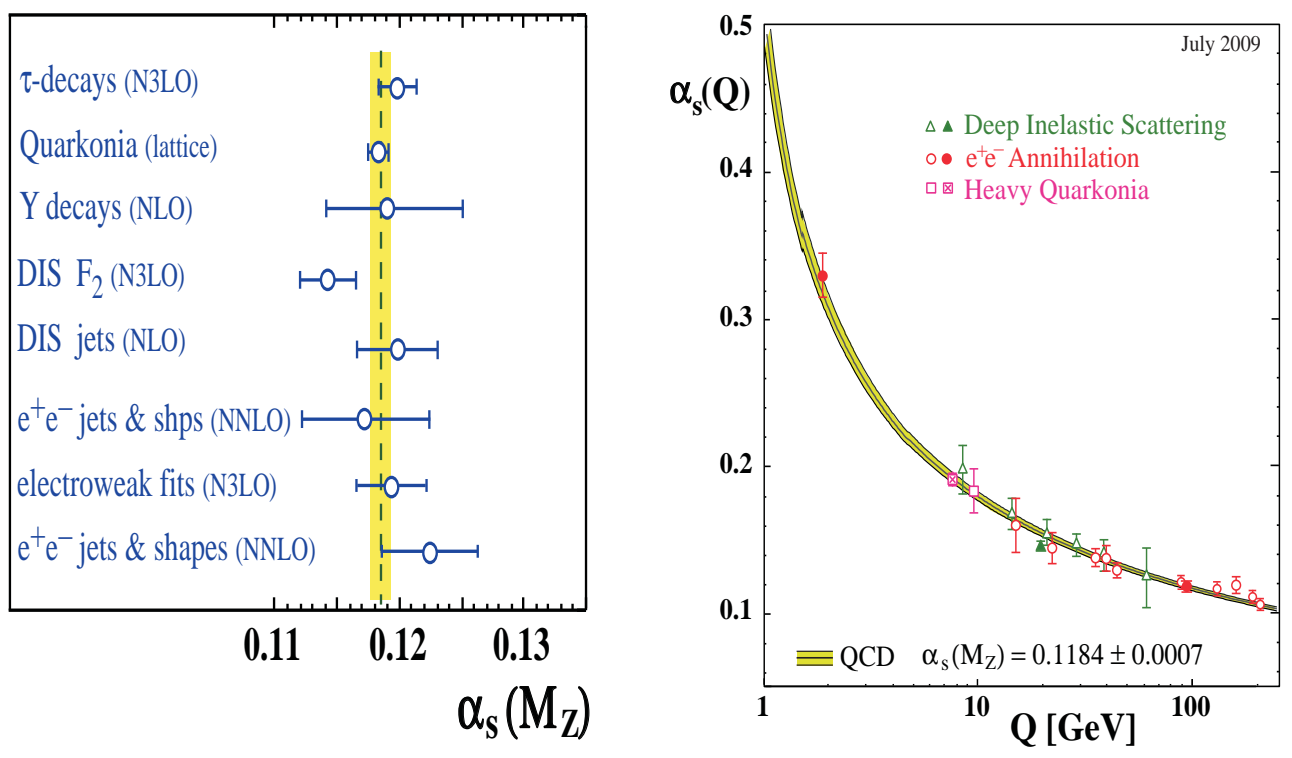

Fig. 32. Left-hand frame: Summary of measurements of $\alpha_{s}\left(M_{Z}\right)$, used as input for the world average value. Right-hand frame: Evolution of the QCD coupling with energy. Among other methods, analyses based on hadron production in $e^{+} e^{-}$annihilation play a leading role up to the highest LEP energies. Both frames are from Bethke 228].

and QCD - is now a diagnostic tool to probe physics at scales as high as the the grand unification scale.

\section{Jets as tools}

In the preceding sections, we discussed the impact which jet physics in $e^{+} e^{-}$annihilation experiments had in establishing QCD quantitatively. This progress owes itself to some extent to the fact that in $e^{+} e^{-}$annihilation the initial state is precisely known. This is not the case in other high energy collisions, such as the electroand photoproduction processes $e p$ and $\gamma p$, as well as the gamma-gamma and hadron hadron collisions, $\gamma \gamma, p \bar{p}$ and $p p$. Here, jets could be used as powerful tools for studying other aspects of high energy collisions. Examples are the partonic composition of the proton, i.e., quark and gluon densities of the proton (and antiproton), the parton distribution functions (PDFs) of the photon and the QCD coupling at HERA, Tevatron and the LHC. Yet other applications of jet physics include analyses of the electroweak sector and searches for new heavy particles in many extensions of the Standard Model (SM) - the QCD and electroweak theory of particle physics based on the groups $S U(3)_{c} \otimes S U(2)_{I} \otimes U(1)_{Y}$ Thus the prominent role of jets in studying QCD phenomena extends to quite different areas in particle physics.

Before we embark upon illustrating the use of jets as tools in $e p, \gamma \gamma, p \bar{p}$ and $p p$ collisions, it is worth pointing out that in these processes, QCD is at work in both the initial and the final states as opposed to the $e^{+} e^{-}$annihilation processes, where it influences only the final state distributions and rates. As seen in Fig. 33 for the DIS process, the cross section depends on three components: (i) the probability of finding a parton in the proton having a fractional longitudinal momentum $x$ (or $x_{\mathrm{Bj}}$ ), (ii) the interaction between these partons and the virtual photon, and (iii) the transition of partons to jets, which theoretically involved the recombination of two partons into 
one jet. While perturbative QCD provides a framework to evolve the PDFs and the fragmentation functions FFs from a low scale $\mu^{2}=Q_{0}^{2}$ to a high scale $\mu^{2}=Q^{2}$, non-perturbative inputs for the PDFs and FFs are required at the lower scale. This is obtained by parametrising an ansatz at lower scale. The theoretical tool which enables this is called factorization, a key concept in the application of QCD to high energy processes 229]. Simply stated, factorization of a process (such as inclusive- or jetcross sections in deep inelastic scatterings) allows it to be expressed as the product of a short-distance part, calculable in perturbative QCD, and a long distance part, comprising of non-perturbative matrix elements or PDFs. The universality of the PDFs and the evolution from a lower scale to a higher scale are process-independent. The division into the short- and long-distance parts is governed by the factorization scale $\mu_{F}$. We illustrate the applications of factorization on the example of deep inelastic scattering processes, discussed below.

\section{1 ep Collisions}

In DIS, described here by the process $e p \rightarrow e+X$, an electron $e$ with momentum $k$ emits an off-shell photon with momentum $q$ which interacts with a proton of momentum $P$. For virtualities of the photon $\left(Q^{2}=-q^{2}>0\right)$ far above the squared proton mass (but far below the $Z$-boson mass), the differential cross section in terms of the kinematic variables $Q^{2}, x=Q^{2} /(2 P . q)$ and $y=(q . P) /(k . P)$ is

$$
\frac{d^{2} \sigma}{d x d Q^{2}}=\frac{4 \pi \alpha}{2 x Q^{2}}\left[\left(1+(1-y)^{2}\right) F_{2}\left(x, Q^{2}\right)-y^{2} F_{L}\left(x, Q^{2}\right)\right]
$$

where $F_{2}\left(x, Q^{2}\right)$ and $F_{L}\left(x, Q^{2}\right)$ are proton structure functions, which encode the interaction between the photon and the proton. The structure functions are not calculable in perturbative QCD. In the lowest order, i.e., keeping only the Born contribution as shown in Fig. 33 (a) where $x=x_{\mathrm{Bj}}$, the structure functions are given by

$$
F_{2}\left(x, Q^{2}\right)=x \sum_{q} e_{q}^{2} f_{q / p}(x): F_{L}\left(x, Q^{2}\right)=0,
$$

where $f_{q / p}(x)$ is the PDF for quarks of type $q$ inside the proton. This result in which $f_{q / p}(x)$ are independent of the scale $Q$ is the "quark-parton model" picture. Hence, in this picture, also the structure functions $F_{2}$ and $F_{L}$ are independent of $Q^{2}$. Including higher order perturbative QCD corrections, the structure function $F_{2}\left(x, Q^{2}\right)$ has the form 230 ]

$$
F_{2}\left(x, Q^{2}\right)=x \sum_{n=0}^{\infty} \frac{\alpha_{s}^{n}\left(\mu_{R}^{2}\right)}{(2 \pi)^{n}} \int_{x}^{1} \frac{d z}{z} C_{2, i}^{(n)}\left(z, Q^{2}, \mu_{F}^{2}, \mu_{R}^{2}\right) f_{i / p}\left(\frac{x}{z}, \mu_{F}^{2}\right)
$$

Here we have a series in powers of $\alpha_{s}\left(\mu_{R}^{2}\right)$, each term involving a coefficient $C_{2, i}^{(n)}$, which can be calculated using Feynman graphs. The scale $\mu_{R}$ is called the renormalization scale at which the QCD coupling constant $\alpha_{s}\left(\mu_{R}^{2}\right)$ is calculated. The leading order in $\alpha_{s}$ QCD Compton scattering and the boson-gluon fusion contributions are shown in Fig. 33 (b) and 33 (c), respectively. An important point to note is that the momentum of the quark when it interacts with the photon and the momentum when the quark is extracted from the proton may differ. The ratio of these two momenta is $z$. The $C_{2, i}^{(n)}$ coefficients depend on the ratio $z$, and hence one has to integrate over $z$ as indicated above. In lowest order, i.e. without including any perturbative QCD corrections, one 


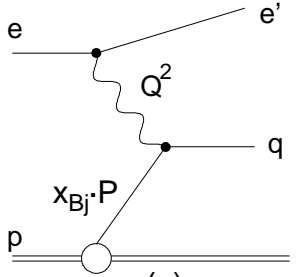

(a)

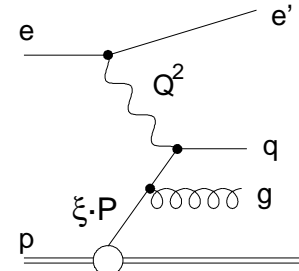

(b)

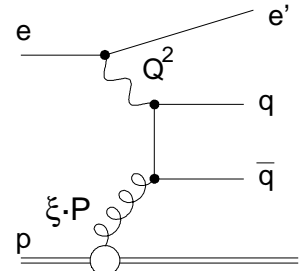

(c)

Fig. 33. Deep-inelastic lepton-proton scattering at different orders in $\alpha_{s}$ : (a) Born contribution $\mathcal{O}(1)$, (b) example of the QCD Compton scattering $\mathcal{O}\left(\alpha_{s}\right)$ and (c) boson-gluon fusion $\mathcal{O}\left(\alpha_{s}\right)$.

has $C_{2, q}^{(0)}=e_{q}^{2} \delta(1-z)$ and $C_{2, g}^{(0)}=0$, and one recovers the "quark-parton model" result.

The PDFs $f_{i / p}\left(\frac{x}{z}, \mu_{F}^{2}\right)$ depend on the factorisation scale $\mu_{F}^{2}$, and this dependence is governed by the DGLAP equation. In leading order in $\alpha_{s}\left(\mu_{F}^{2}\right)$, this reads as follows

$$
\frac{\partial f_{i / p}\left(x, \mu_{F}^{2}\right)}{\partial \mu_{F}^{2}}=\sum_{j} \frac{\alpha_{s}\left(\mu_{F}^{2}\right)}{2 \pi} \int_{x}^{1} \frac{d z}{z} P_{i \rightarrow j}^{(1)}(z) f_{j / p}\left(\frac{x}{z}, \mu_{F}^{2}\right)
$$

where, for example, $P_{q \rightarrow g}^{(1)}(z)=T_{R}\left(z^{2}+(1-z)^{2}\right)$, and the others can be extracted from Eq. (51). The coefficient functions are also $\mu_{F}$-dependent, and in the leading order in $\alpha_{s}$, one has $C_{2, i}\left(x, Q^{2}, \mu_{R}^{2}, \mu_{F}^{2}\right)=C_{2, i}\left(x, Q^{2}, \mu_{R}^{2}, Q^{2}\right)-\ln \left(\frac{\mu_{F}^{2}}{Q^{2}}\right) \sum_{j} \int_{x}^{1} \frac{d z}{z} P_{i \rightarrow j}^{(1)} C_{2, j}^{(0)}\left(\frac{x}{z}\right)$. In the above expressions, the choice of the renormalization and factorization scales is arbitrary. Varying $\mu_{F}$ and $\mu_{R}$ provides an estimate of the scale-dependent uncertainties. In inclusive DIS processes, the default choice is $\mu_{R}=\mu_{F}=Q$.

The extension of the factorization formalism to the processes with two initial-state hadrons follows very much along the same lines, and we shall discuss this somewhat later, as we discuss high energy hadronic collisions.

\subsubsection{Jets in DIS processes}

Jet production in neutral current (NC) deep inelastic scattering at the HERA collider at DESY provides an important further testing ground for QCD. While inclusive DIS gives indirect information on the strong coupling via scaling violations of the proton structure functions, the production of jets allows one a direct measurement of the strong coupling constant $\alpha_{s}$. The Born contribution to DIS (Fig. 33a) generates no transverse momentum in the $\gamma^{*} p$ centre-of-mass frame, where the virtual boson and the proton collide head on. Significant transverse momentum in the $\gamma^{*} p$ frame is produced at leading order (LO) in the strong coupling $\alpha_{s}$ by the QCD-Compton (Fig. 33b) and the photon-gluon fusion (Fig. 33k) processes. In LO the momentum fraction of the proton carried by the parton is given by $\xi=x_{B j}\left(1+M_{12}^{2} / Q^{2}\right)$, where $x_{B j}$ is the Bjorken scaling variable $x_{B j}=Q^{2} /\left(Q^{2}+W^{2}\right)$. Here $W$ is the total c.m. energy $W^{2}=(q+P)^{2}, q=$ is the momentum of the virtual photon, $M_{12}$ is the invariant mass of two jets of highest $p_{T}$ and $Q^{2}$ is the negative four-momentum transfer squared of the ingoing and outgoing electron. In the kinematic region of low $Q^{2}$ and low $\xi$, the $\gamma^{*}$-gluon fusion dominates the jet production imparting sensitivity to the gluon component of the parton density functions (PDFs) of the proton, whereas the contribution of the QCD-Compton process yields information on the various quark (antiquark) components of the proton. 


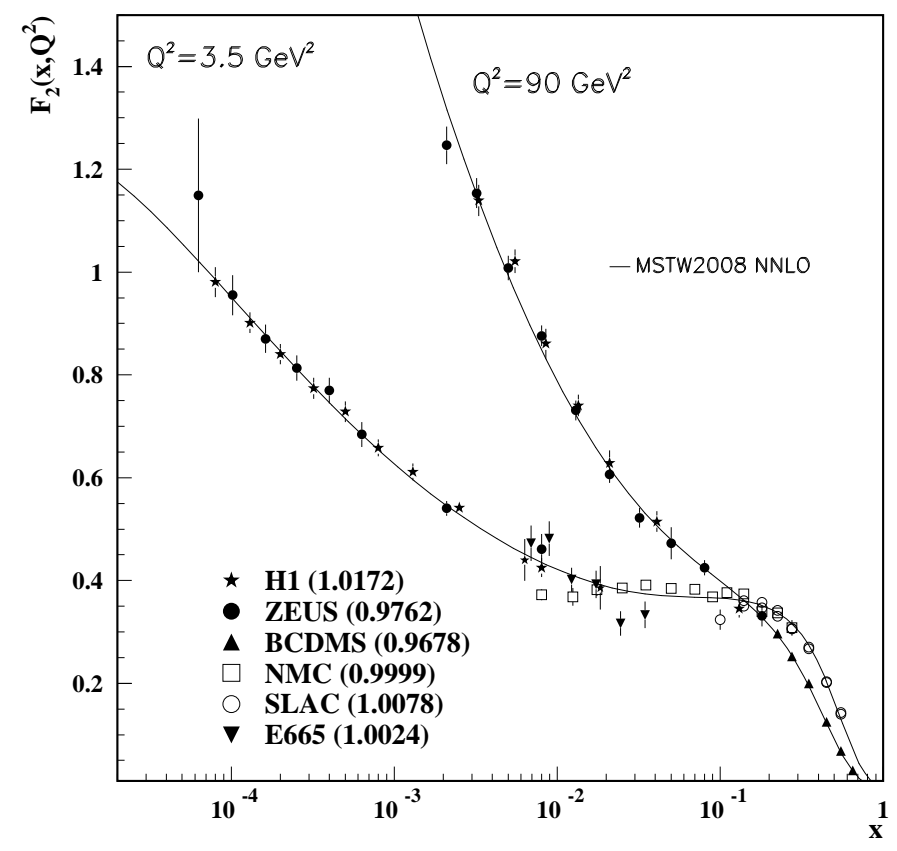

Fig. 34. Proton structure function $F_{2}^{p}\left(Q^{2}, x\right)$ given at two $Q^{2}$ values $\left(3.5 \mathrm{GeV}^{2}\right.$ and 90 $\mathrm{GeV}^{2}$ ). The various data sets have been normalised by the factors shown in the brackets in the key to the plot, which were determined in the NNLO MSTW2008 global analysis [232. (From Amsler et al. in Ref. 235]).

In order to make theoretical predictions on jet productions in neutral current DIS scattering one needs the PDFs of the proton, provided mostly by the global analysis collaborations (i.e., analysis in which all high energy physics measurements relevant for testing QCD and determination of various parameters and non-perturbative functions are undertaken), such as CTEQ 231 and MSTW 232. In addition one must have infrared and collinear safe parton cross sections, which are known now up to NLO in $\alpha_{s} 233234$. An example of the inclusive DIS measurements at HERA together with data from lower energy fixed target experiments is shown in Fig. 34. A striking feature of the HERA data is the dramatic rise of the proton structure function $F_{2}\left(x, Q^{2}\right)$ for increasing $Q^{2}$ and low values of $x$ (typically $x \leq 10^{-2}$ ). Almost all of this rise of $F_{2}\left(x, Q^{2}\right)$ is due to the gluon density in the proton. This has profound consequences for high energy $p \bar{p}$ (at the Tevatron) and $p p$ collisions (at the LHC).

DIS jet production depends in general on two large scales $Q=\sqrt{Q^{2}}$ and the $p_{T}$ of the produced jets. The $e p$ jet data were collected by two detectors at HERA: H1 and ZEUS, resulting from the collision of electrons or positrons with energy $E_{e}=27.6$ $\mathrm{GeV}$ with protons of energy $E_{p}=920 \mathrm{GeV}$, providing a center-of-mass energy of $\sqrt{s} \simeq 320 \mathrm{GeV}$. In the more recent analysis the inclusive $k_{T}$ jet algorithm [79] is used to combine the particles in the hadronic final state into jets. Theoretical predictions (at next-to-leading order) have been corrected for hadronisation effects, which are calculated via Monte Carlo Models with parton showers. The most recent publication on jet production in DIS comes from the H1 collaboration at HERA, where data up to 

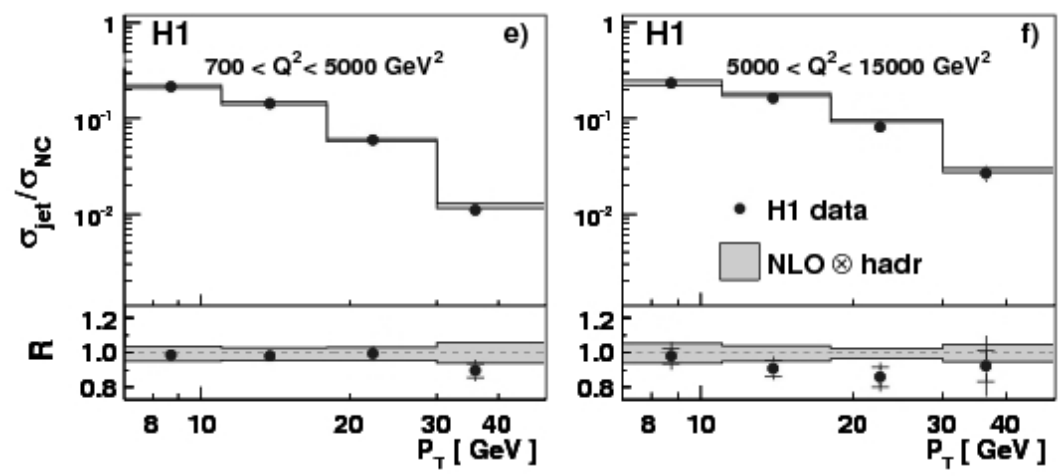

Fig. 35. The normalised inclusive jet cross sections measured as a function of the jet transverse momentum in the Breit frame $P_{T}$ in two regions of $Q^{2}$ indicated on the frames. The points are shown at the average value of $P_{T}$ within each bin. (From H1 Collaboration 236]).

2007 are included and $Q^{2}$ spans the range $150<Q^{2}<15000 \mathrm{GeV}^{2}$ 236. Inclusive jet, 2 -jet and 3-jet cross sections, normalised to the neutral current (NC) deep inelastic scattering cross section, are measured as functions of $Q^{2}$, jet transverse momentum and proton momentum fraction. We show in Fig. 35 the normalised inclusive jet cross section as a function of the jet transverse momentum $p_{T}$ in the Breit frame (defined as the frame in which $2 x \mathbf{p}+\mathbf{q}=0$, where $\mathbf{p}$ and $\mathbf{q}$ are the 3 -momenta of the proton and virtual photon, respectively) for two ranges of $Q^{2}, 700<Q^{2}<5000$ $\mathrm{GeV}^{2}$ (shown in the left-hand frame) and $5000<Q^{2}<15000 \mathrm{GeV}^{2}$ (shown in the right-hand frame). Agreement between data and theoretical predictions 233234] is excellent. The ratio $\mathrm{R}$ (of data over theory) lies near 1 (shown at the bottom of these frames). Similar plots for bins with smaller $Q^{2}$ can be seen in [236]. HERA data on inclusive jet production in DIS 237 238 239 have constrained the gluon density in the range $0.01<x<0.1$ The strong coupling $\alpha_{s}\left(Q^{2}\right)$ has been determined and translated into $\alpha_{s}\left(M_{Z}\right)=0.1168 \pm 0.0007$ (exp. $)_{-0.0036}^{+0.0046}$ (theor.) \pm 0.0016 (PDF) using the usual renormalisation group equation. This result is competitive with those from $e^{+} e^{-}$data and is in good agreement with the world average [235.

A similar recent analysis of the ZEUS collaboration has less integrated luminosity, as it is based only on the data taken from 1998- 2000. However, their data include also results for rather large $Q^{2}$. Therefore, $Z$ exchange is included in addition to the $\gamma$-exchange. The analysis is done in a similar fashion as that of the H1 collaboration described above. They also studied the inclusive one-jet cross section as a function of $Q^{2}$ and $E_{T, B}^{\text {jet }}$ (the transverse energy of the jet in the Breit frame). In addition they also measured this cross section for three different radii, $R=0.5,0.7$ and 1.0, used for combining hadrons into jets with the help of the inclusive $k_{T}$ cluster algorithm 79180 . The results are shown in Fig. 36 for $d \sigma / d Q^{2}$ for $E_{T, B}^{j e t}>8 \mathrm{GeV}$ as a function of $Q^{2}$. Further kinematic constraints, namely $\left|\cos \gamma_{h}\right|<0.65$, where $\gamma_{h}$ corresponds to the angle of the scattered quark in the quark-parton model and is constructed using the hadronic final state, and the pseudorapidity range $-2<\eta_{B}^{\text {jet }}<1.5$, are indicated on the figure. The pseudorapidity variable, defined as $\eta=-\ln \tan (\theta / 2)$ where $\cos \theta=p_{z} /|p|$, is approximately equal to the (longitudinal-boost-invariant) variable called rapidity $y=\frac{1}{2} \ln \left(\frac{E+p_{z}}{E-p_{z}}\right)$ in the limit $|p| \gg m$, and can be measured when the mass $m$ and the momentum of the particle $|p|$ are unknown. The NLO QCD predictions with scales $\mu_{R}=E_{T, B}^{j e t}, \mu_{F}=Q$, corrected 


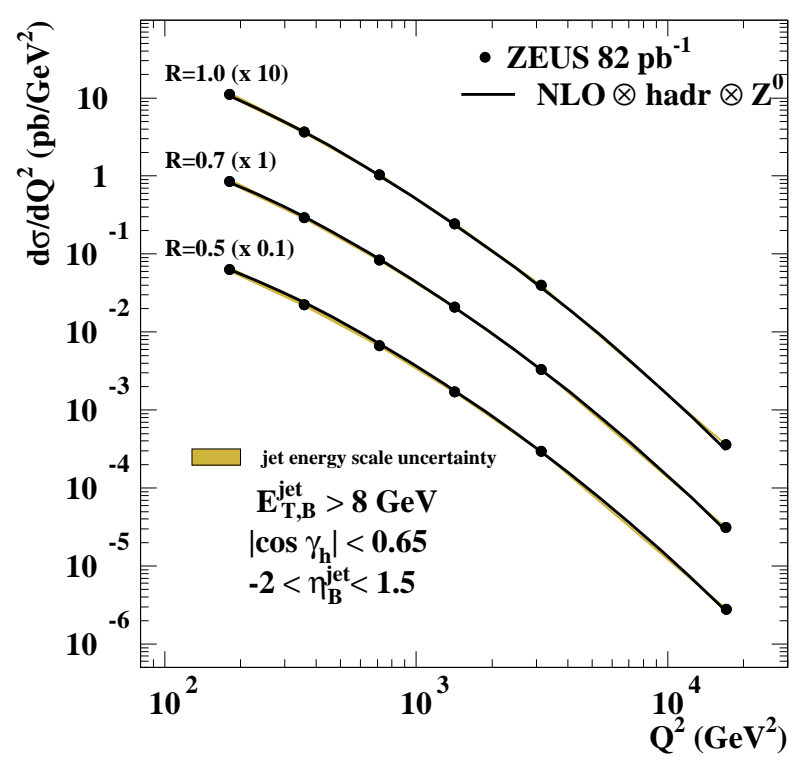

Fig. 36. Measured differential cross-section $d \sigma / d Q^{2}$ for inclusive-jet production with $E_{T, B}^{\text {jet }}>8 \mathrm{GeV}$ and $-2<\eta_{\mathrm{B}}^{\text {jet }}<1.5$ (dots) for different jet radii, in the kinematic range given by $\left|\cos \gamma_{h}\right|<0.65$. (From ZEUS Collaboration [240]).

to include hadronisation and $Z$ effects, are compared with the measurements 240. The calculations reproduce the measured differential cross section quite well for all three jet radii considered. In this work also $\alpha_{s}\left(Q^{2}\right)$ has been determined. The result is $\alpha_{s}\left(M_{Z}\right)=0.1207 \pm 0.0014$ (stat. $)_{-0.0033}^{+0.0035}$ (exp. $)_{-0.0023}^{+0.0023}$ (theor.), which is also consistent with the world average.

We now turn to photoproduction. At HERA the largest cross section is due to photoproduction, where the beam (electron or positron) interacts with the proton via the exchange of a virtual photon with a small virtuality $Q^{2} \approx 0$. The spectrum of the ingoing virtual photon can very well be described by the well-known WeizsäckerWilliams formula 241242243$]$.

The photoproduction of single jets, dijets and triple jets with high transverse momenta can be calculated also within perturbative QCD if the transverse momentum of the jets is large enough to provide the hard scale. Besides the larger cross section, as compared to the DIS jet production, the photoproduction of jets does not depend on the additional scale $Q$. The contributions to the theoretical cross sections which have been calculated up to NLO come from two processes: (i) the direct process in which the photon enters the hard sub-processes directly by coupling to the quarks, in the same way as in deep-inelastic ep scattering (see Fig. 33b, c in LO), and (ii) the socalled resolved process in which the photon fluctuates into partons, quarks or gluon, 
and one of them participates in the hard parton-parton scattering process 244245 . This latter process is equivalent to jet production in hadron-hadron collisions, of which the LO hard scattering cross sections for $q q^{\prime} \rightarrow q q^{\prime}, g g \rightarrow g g$ and $g q \rightarrow g q$ are written below. The only difference is that the PDFs of one of the hadrons is replaced by the photon PDF. This process, therefore, is sensitive to the parton structure of the proton and the photon. It is one of the few processes which can give information on the gluon content of the photon.

The basic $\gamma$-parton processes which enter the calculation of the direct process in LO are the following: QCD Compton process: $\gamma q \rightarrow g q$, and the photon-gluon fusion: $\gamma g \rightarrow q \bar{q}$. These $\gamma$-parton cross sections have the following simple forms

$$
\begin{gathered}
\gamma q \rightarrow g q: \frac{d \sigma}{d \cos \theta^{*}} \sim e_{q}^{2} \frac{\alpha}{\pi} \frac{\alpha_{s}}{\pi} \frac{1}{s} \frac{4}{9}\left(-\frac{\hat{u}}{\hat{s}}-\frac{\hat{s}}{\hat{u}}\right), \\
\gamma g \rightarrow q \bar{q}: \frac{d \sigma}{d \cos \theta^{*}} \sim e_{q}^{2} \frac{\alpha}{\pi} \frac{\alpha_{s}}{\pi} \frac{1}{2 s}\left(\frac{\hat{u}}{\hat{t}}+\frac{\hat{t}}{\hat{u}}\right),
\end{gathered}
$$

where $e_{q}$ is the charge of the quark with flavour $q, \hat{s}=4, \hat{t}=-2\left(1-\cos \theta^{*}\right), \hat{u}=$ $-2\left(1+\cos \theta^{*}\right)$ and $\theta^{*}$ is the angle of the dijets in their centre-of mass system. $\left|\cos \theta^{*}\right|$ is related to the pseudorapidities of the two jets, $\eta_{1}$ and $\eta_{2}$ by

$$
\left.\left|\cos \theta^{*}\right|=\mid \tanh \left(\eta_{1}-\eta_{2}\right) / 2\right) \mid \text {. }
$$

There are many observables which have been measured and which can be used to test the basic parton-parton cross sections for the direct and resolved process up to NLO $246 \mid 247248$ 249. We shall present only a few taken from the most recent H1 250] and ZEUS 251 publications. The $d \sigma / d\left|\cos \theta^{*}\right|$ distribution has been studied as a function of $\left|\cos \theta^{*}\right|$ by the $\mathrm{H} 1$ collaboration [250] with and without an additional cut on the invariant mass of the two jets $M_{j j}$ for the direct (resolved) enhanced contribution. This analysis is done in terms of a variable $x_{\gamma}$ defined by,

$$
x_{\gamma}=\frac{1}{2 E_{\gamma}} \sum_{i=1}^{2} E_{T, i} e^{-\eta_{i}},
$$

where $E_{T, 1}$ and $E_{T, 2}$ are the transverse energies of the two jets with the two largest $E_{T}$ 's. In LO the direct contribution is at $x_{\gamma}=1$ and the resolved contribution has $x_{\gamma}<1$. Therefore, by selecting events with $x_{\gamma}>0.8\left(x_{\gamma}<0.8\right)$ the direct (resolved) parts of the cross sections are dominant. The results of the H1 analysis are shown in Fig. 37 for the two bins of $x_{\gamma}$ as a function of $\cos \theta^{*}$, with the upper two frames without a cut on $M_{j j}$ and the lower two frames with $M_{j j}>65 \mathrm{GeV}$.

The cross section $d \sigma / d\left|\cos \theta^{*}\right|$ with the $M_{j j}$ cut is sensitive to the dynamics of the underlying $\gamma$-parton and parton-parton hard interactions. The cross section in the resolved sample $x_{\gamma}<0.8$ rises more rapidly with $\left|\cos \theta^{*}\right|$ than that in the direct sample due to the dominance of the virtual gluon exchange in the resolved processes (see formulae for parton-parton cross sections below). The dependence on $\left|\cos \theta^{*}\right|$ and also the absolute normalisation are well predicted by the NLO calculations. Similar results have been presented by the ZEUS collaboration [252] by varying the dijet mass $M_{j j}$ and their results have been presented in [253]. Another example is the cross section $d \sigma / d \overline{E_{T}}$, where $\overline{E_{T}}$ is the mean transverse energy of the two jets

$$
\overline{E_{T}}=\frac{1}{2}\left(E_{T}^{j e t 1}+E_{T}^{j e t 2}\right) .
$$

An example of such a cross section for $x_{\gamma}>0.75$ and $x_{\gamma}<0.75$, respectively, as presented by the ZEUS collaboration [251] is shown in Fig. 38. The cross section is 

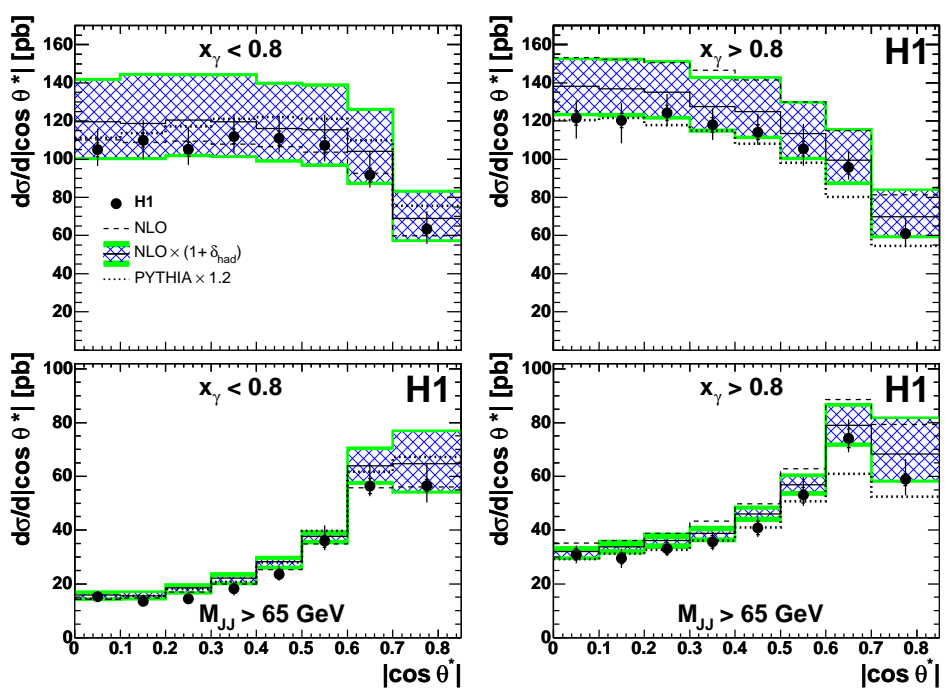

Fig. 37. Bin averaged cross sections as a function of $\left|\cos \theta^{*}\right|$ for data (points), NLO QCD calculations with (solid line) and without (dashed line) hadronisation corrections $\delta_{\text {had }}$ and for the PYTHIA Monte Carlo predictions (dotted line) scaled by a factor of 1.2. The inner (hatched) band of the $\mathrm{NLO} \times\left(1+\delta_{\text {had }}\right)$ result is the scale uncertainty, the outer (shaded) band is the total uncertainty. The cross sections are shown for two regions in $x_{\gamma}$, with and without an additional cut applied on the invariant dijet mass ( $\left.M_{\mathrm{JJ}}\right)$. (From H1 Collaboration [250]).

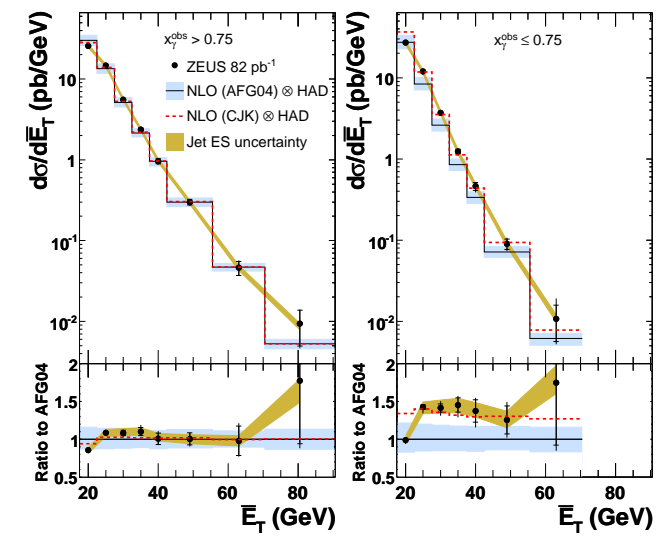

Fig. 38. Measured cross-section $d \sigma / d \bar{E}_{T}$ for (a) $x_{\gamma}^{\text {obs }}>0.75$ and (b) $x_{\gamma}^{\text {obs }} \leq 0.75$ compared with NLO QCD predictions using the AFG04 254 (solid line) and CJK 255] (dashed line) photon PDFs. The predictions using AFG04 are also shown with their associated uncertainties (shaded histogram). The ratios to the prediction using the AFG04 photon PDF are shown at the bottom of the figure. (From ZEUS Collaboration 251]). 
measured up to $\overline{E_{T}} \simeq 80 \mathrm{GeV}$, i.e. further out in $E_{T}$ than in DIS jet production. In this figure also results for two different photon PDFs (namely, the so-called AFG04 [254] and the CJK 255]) are shown. The most sensitive cross section concerning direct and resolved separation is the cross section $d \sigma / d x_{\gamma}^{o b s}$, where $x_{\gamma}^{o b s}$ is the $x_{\gamma}$ defined above with the sum over the two jets (therefore "obs" in the notation of $x_{\gamma}$ since not all jets are included in the sum). The result from the ZEUS collaboration is shown in Fig. 39, from which one can see how the data compare with different photon PDFs assumed in the NLO prediction. An appreciable dependence on these PDFs is seen in the small $x_{\gamma}$ region as one would expect. In an earlier analysis the ZEUS collaboration determined also the strong coupling $\alpha_{s}$, just from jet production in $\gamma p$ interactions alone. The result is $\alpha_{s}\left(M_{Z}\right)=0.1224 \pm 0.0001$ (stat.) ${ }_{-0.0019}^{+0.0022}$ (exp. $)_{-0.0042}^{+0.0054}$ (theor.) [256], and the variation of $\alpha_{s}$ with the scale $\overline{E_{T}}$ has been found in good agreement with the running of $\alpha_{s}$ as predicted by QCD.

Summarising the DIS and photoproduction processes at HERA, we see that QCD and jets have made very significant impact on the profile of the proton and the photon in terms of their respective PDFs, which determine the luminosity functions of the parton-parton scatterings at high energies and hence the hard scattering cross sections of interest. Detailed studies of $F_{2}\left(x, Q^{2}\right)$ at HERA have also rekindled theoretical interest in the small- $x$ region. The evolution in $\ln (1 / x)$ at fixed value of $Q^{2}$ is governed by the so-called BFKL equation 261262. Originally developed to study Regge processes in high energy scatterings and the Pomeranchuk singularity (the QCD Pomeron), it can be combined with the DGLAP equation (for evolution in $Q^{2}$ ) to provide a quantitative description of the DIS structure functions over an enlarged $\left(x, Q^{2}\right)$ domain. Several proposals in carrying out the small- $x$ resummation have been considered in the literature, which are comparatively discussed in a recent working group report 263. In addition, the evolution in $\ln (1 / x)$ leads to soft gluon enhancements, generating a dense gluonic system over a limited range of the nucleon wave function (hot spots). As the gluon occupation number becomes of order $1 / \alpha_{s}$, non-linear effects present in the QCD Lagrangian become important, leading eventually to the saturation of the gluon density in the nucleons in high energy collisions 264. This picture of high energy nucleonic wave functions (a high density, nonperturbative gluonic system with a weak coupling constant) is called the Color Glass Condensate 265, and is of great interest in understanding the QCD aspects of heavy ion collisions, such as at RHIC and the LHC [266].

\section{$7.2 \gamma \gamma$ collisions}

Another area in which jet production has been studied experimentally and theoretically is photon-photon collisions in the LEP2 energy range. The two incoming photons are produced in $e^{+} e^{-}$collisions in the anti-tagged mode, i.e. when both the scattered electron and the positron escape detection. This is kinematically analogous to the photoproduction process in high energy ep collisions at HERA. In $\gamma \gamma \rightarrow$ hadrons, four classes of events have to be distinguished (see, Fig. 40). The variables used in the classification of these events $x_{\gamma}^{+}$and $x_{\gamma}^{-}$, which are analogues of the variable $x_{\gamma}$ in $\gamma p$ collisions, are defined as follows:

$$
\begin{aligned}
& x_{\gamma}^{+}=\frac{\sum_{\text {jets }}\left(E_{\text {jet }}+p_{z, \text { jet }}\right)}{\sum_{\text {part }}\left(E_{\text {part }}+p_{z, \text { part }}\right)}, \\
& x_{\gamma}^{-}=\frac{\sum_{\text {jets }}\left(E_{\text {jet }}-p_{z, \text { jet }}\right)}{\sum_{\text {part }}\left(E_{\text {part }}-p_{z, \text { part }}\right)},
\end{aligned}
$$




\section{ZEUS}

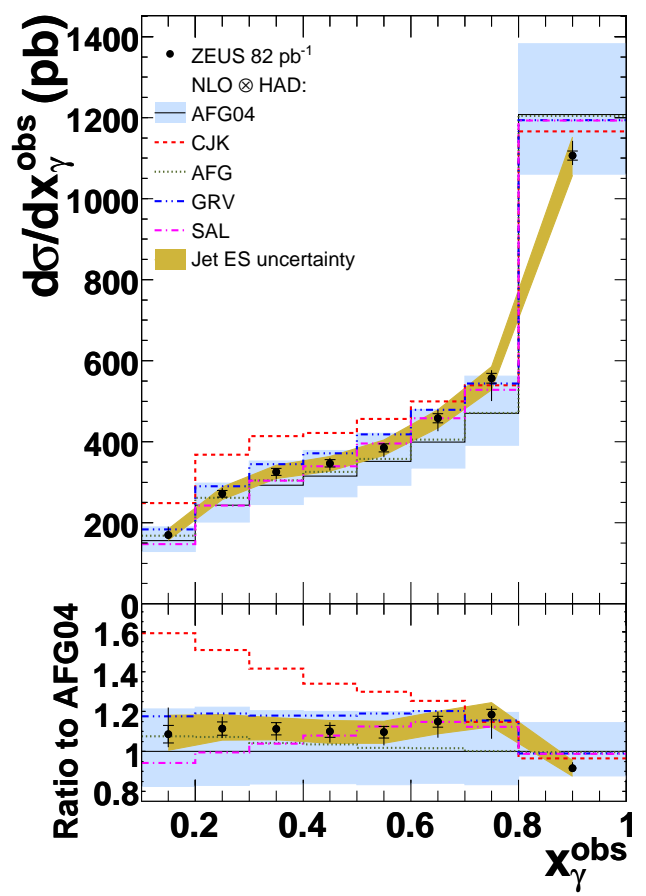

Fig. 39. Measured cross section for $d \sigma / d x_{\gamma}^{\text {obs }}$ compared with the NLO QCD predictions using the AFG04 254] (solid line), CJK 255] (dashed line), AFG 257] (dotted line), GRV 258 259 (dashed and double-dotted line) and SAL 260 (dashed and single-dotted line) photon PDFs. The ratios to the prediction using the AFG04 254] photon PDF are shown at the bottom of the figure. (From 251).

where 'part' corresponds to all detected particles and $E_{\text {jet }}$ and $p_{z \text {,jet }}$ are the two hardjets energy and the component of jet momentum along the $z$-axis, respectively. The four classes are:

1. Hadron production via vector meson interactions (Vector-Meson Dominance Model VDM) [267/268] (Fig. 406).

2. The direct domain, where both $x_{\gamma}^{+}$and $x_{\gamma}^{-}$are close to 1 . This domain is mostly populated by the quark-parton model like events $\gamma \gamma \rightarrow q+\bar{q}$ (Fig. 40b).

3. The single resolved domain, with the presence of a remnant jet, where only one of the $x_{\gamma}^{+}$and $x_{\gamma}^{-}$is close to 1 and the other is shifted to some lower value (Fig. 40k).

4. The double-resolved domain, where both $x_{\gamma}^{ \pm}$are shifted to values below 1 (Fig. 40 d).

Due to the appearance of the double resolved region, jet production in $\gamma \gamma$ collisions has increased sensitivity to the gluon content of the resolved photon. This has enormous significance for future high energy $\gamma-\gamma$ collisions, being entertained in the context of a high energy linear $e^{+} e^{-}$collider.

In the past, dijet production in $\gamma \gamma$ collisions has been studied experimentally at $\sqrt{s_{e e}}$ from 189 to $209 \mathrm{GeV}$ by the OPAL [269] and DELPHI [270] collaborations at LEP. To that end a number of observables (differential distributions) have been measured by introducing $x_{\gamma}^{ \pm}$-cuts at 0.75 (OPAL) and $x_{\gamma}^{ \pm}=0.85$ (DELPHI). These 

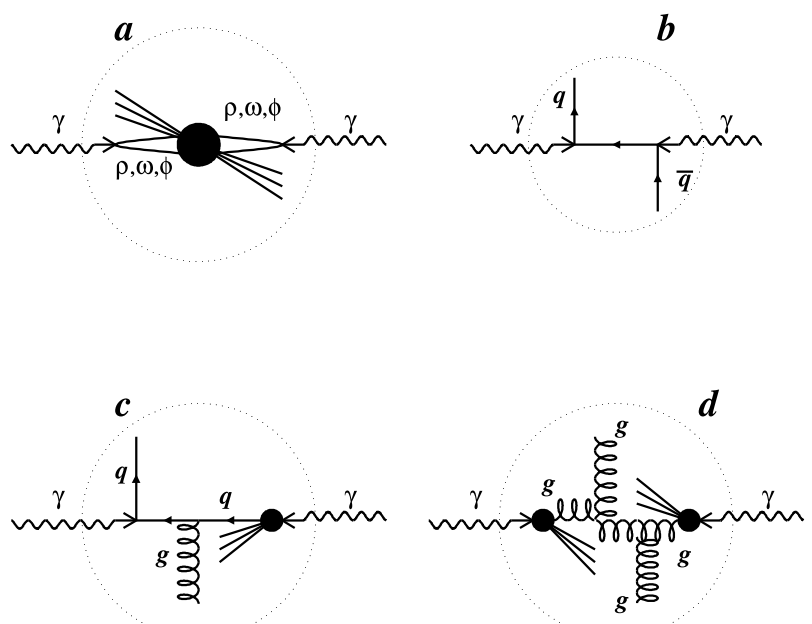

Fig. 40. Main diagrams corresponding to the hadron production in $\gamma \gamma$ collisions via vector meson interactions (VDM-like, a), point-like interactions (QPM-like, b) and with one (c) or both (d) photons resolved into partons. (From [270]).

distributions include, among others, $d \sigma_{\text {dijet }} / d \bar{E}_{T}^{\text {jet }}$, with $\bar{E}_{T}^{\text {jet }}=1 / 2\left(E_{T, 1}^{\text {jet }}+E_{T, 2}^{\text {jet }}\right)$ and $d \sigma_{\text {dijet }} / d x_{\gamma}$. The data from both collaborations have been compared with the NLO QCD calculations based on the work of [246] and are found to be in good agreement. This is shown in Fig. 41 for the differential distribution $f \dot{d} \sigma / d \bar{E}_{T}^{\text {jet }}$, where the factor $f$ is used to visibly separate the three measurements. Inclusive jet production in $\gamma-\gamma$ collisions has also been measured by the L3 [271] and OPAL [272] collaborations at LEP.

\subsection{Proton colliders}

\subsubsection{Fundamental QCD scattering processes}

In parallel to electron and photon processes in QED, a large number of $2 \rightarrow 2$ scattering processes involving quarks and gluons are predicted in QCD, see e.g. [55]. They give rise to jets at hadron colliders. Most interesting are the fundamental abelian processes in QED transcribed to the non-abelian extensions in QCD, like

$$
\begin{aligned}
\text { Rutherford quark scattering }: & q q^{\prime} \rightarrow q q^{\prime}, \\
\text { Rutherford gluon scattering }: & g g \rightarrow g g, \\
\text { Super - Compton process }: & g q \rightarrow g q .
\end{aligned}
$$

Representative scattering diagrams are depicted in Fig 42, The associated cross sections scale in the energy squared $s$ for massless initial and final-state quarks, while the angular distributions are given by

$$
q q^{\prime} \rightarrow q q^{\prime}: \frac{d \sigma}{d \cos \theta^{*}} \sim\left(\frac{\alpha_{s}}{\pi}\right)^{2} \frac{1}{s} \frac{4}{9} \frac{\hat{s}^{2}+\hat{u}^{2}}{\hat{t}^{2}},
$$




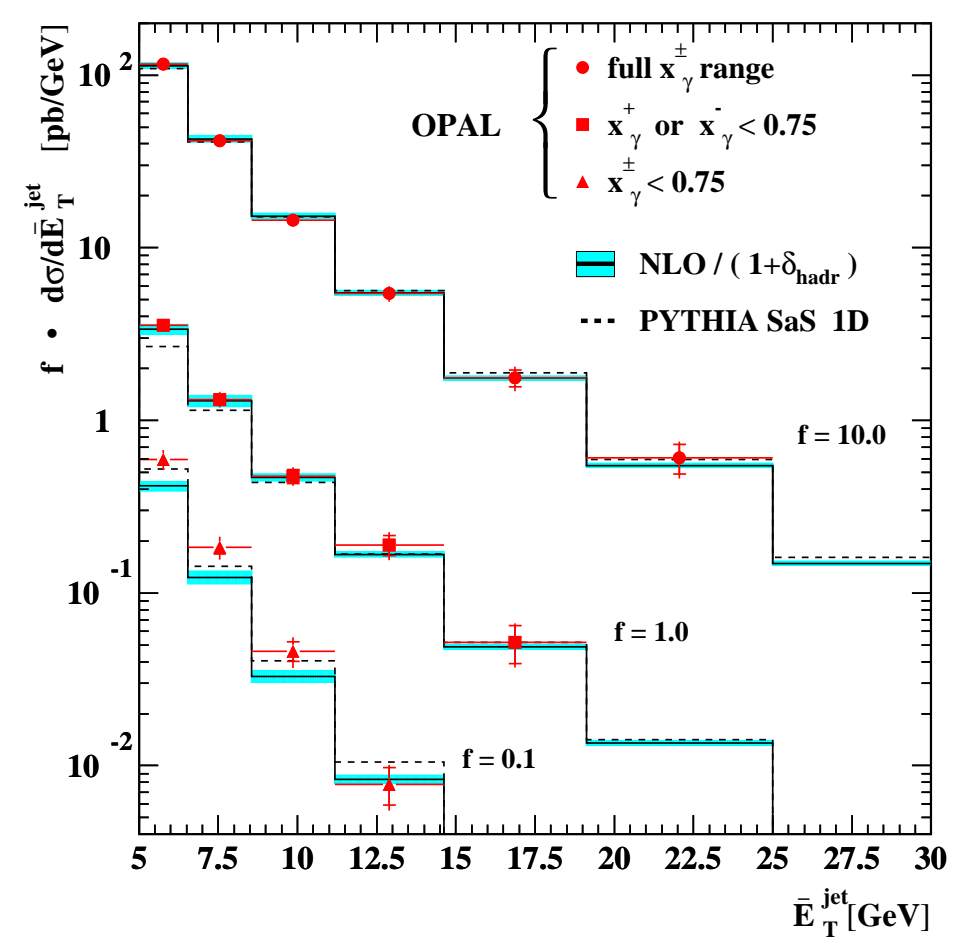

Fig. 41. The dijet cross-section in $\gamma \gamma$ collisions at LEP as a function of the mean transverse energy $\bar{E}_{T}^{\text {jet }}$ of the dijet system, for the three regions in $x_{\gamma}^{+}-x_{\gamma}^{-}$-space given in the figure. The factor $f$ is used to separate the three measurements in the figure more clearly. The prediction of the LO program PYTHIA is compared to the data. The NLO calculation is from 247]. (From OPAL 269]).

$$
\begin{gathered}
g g \rightarrow g g: \frac{d \sigma}{d \cos \theta^{*}} \sim\left(\frac{\alpha_{s}}{\pi}\right)^{2} \frac{1}{s} \frac{9}{2}\left[3-\frac{\hat{s} \hat{u}}{\hat{t}^{2}}-\frac{\hat{s} \hat{t}}{\hat{u}^{2}}-\frac{\hat{t} \hat{u}}{\hat{s}^{2}}\right], \\
g q \rightarrow g q: \frac{d \sigma}{d \cos \theta^{*}} \sim\left(\frac{\alpha_{s}}{\pi}\right)^{2} \frac{1}{s}\left[\frac{\hat{u}^{2}+\hat{s}^{2}}{\hat{t}^{2}}-\frac{4}{9} \frac{\hat{s}^{2}+\hat{u}^{2}}{\hat{s} \hat{u}}\right],
\end{gathered}
$$

and the variables $\hat{s}, \hat{u}$ and $\hat{u}$ have been defined earlier. One should notice the threegluon coupling already in LO. These amplitudes generate the expected Rutherford singularities $\sim d \theta^{* 2} / \theta^{* 4}$ for forward scattering $\hat{t} \rightarrow 0$, and analogously for backward scattering $\hat{u} \rightarrow 0$.

Calculating the experimentally observed cross sections at hadron colliders requires three essential steps, which we have already outlined in the context of calculating the DIS cross sections, namely (i) the hard $2 \rightarrow 2$ scattering processes, including NLO QCD corrections, (ii) flux of the incoming partons, determined in terms of the PDFs of the protons (and antiprotons), discussed earlier in the context of DIS scattering at HERA, and (iii) hadronic (non-perturbative) corrections. Here also QCD plays an important role in terms of the scale dependence of the PDFs and FFs. Thus, for example, the cross section of the hadron-hadron scattering with the four-momenta of 


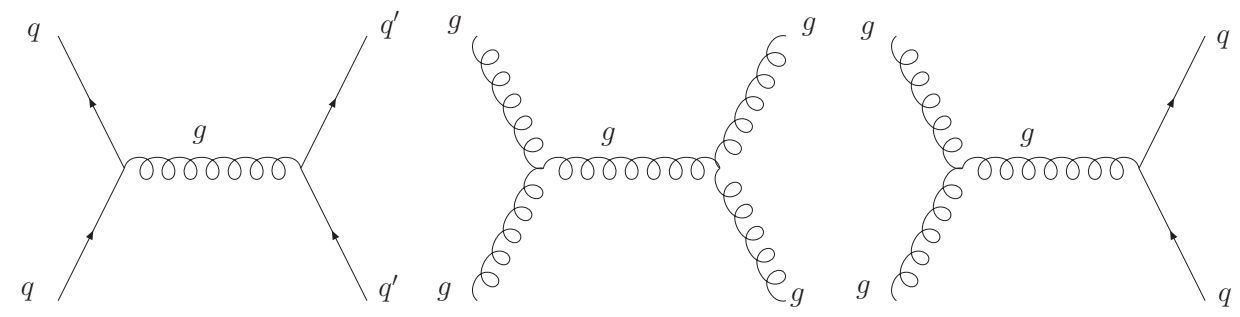

Fig. 42. Representative Feynman diagrams for fundamental QCD processes in hadronic collisions.
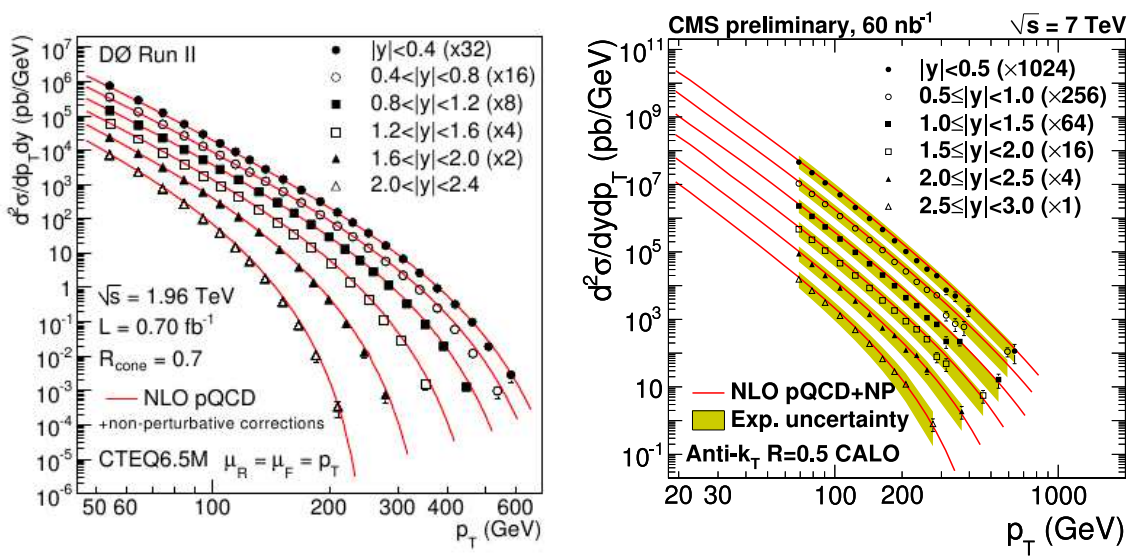

Fig. 43. Left-hand frame: Transverse energy distribution of jets at the Tevatron 273. Righthand frame: Comparison between the measured $p_{T}$ spectra by the CMS collaboration at the LHC and theory predictions for calorimeter jets 274. For better visibility the spectra in both frames are multiplied by arbitrary factors indicated in the legend.

the two colliding hadrons $P_{1}$ and $P_{2}$ can be written as [55]

$\sigma\left(P_{1}, P_{2}\right)=\sum_{i, j} \int d x_{1} \int d x_{2} f_{i}\left(x_{1}, \mu_{F}^{2}\right) f_{j}\left(x_{2}, \mu_{F}^{2}\right) \hat{\sigma}_{i j}\left(p_{1}, p_{2}, \alpha_{s}\left(\mu_{R}^{2}\right), Q^{2} / \mu_{F}^{2}, Q^{2} / \mu_{R}^{2}\right)$,

where the hard interaction between the partons $i$ and $j$ is given by $\hat{\sigma}_{i j}$ and $p_{1}=x_{1} P_{1}$ and $p_{2}=x_{2} P_{2}$ are the momenta of the two partons.

\subsubsection{Jets in hadron colliders and tests of $Q C D$}

An example for inclusive jet production at the Tevatron measured by the D0 collaboration is shown in Fig. 43 (left-hand frame). Similar measurements have been done by the CDF collaboration at the Tevatron. The dominant contribution at small $p_{T}$ can be traced back to Rutherford gluon scattering $g g \rightarrow g g$. This result is naturally expected since, on average, the gluon colour charges are significantly larger than the quark colour charges and, as discussed earlier, the gluon flux for low values of $x$ by far exceeds the quark flux of high-energy protons. These jet cross sections can be exploited to determine the gluon distribution of the proton and to measure the QCD coupling 275. By combination with other measurements the two observables are disentangled in the Tevatron measurements. The gluon flux extracted this way is large, as 
anticipated, and the QCD coupling is compatible with the world average. This [273], and related measurements [276 277] impact on the proton PDFs and have been used in updating this information 231232. In particular, they provide constraints on the gluon (and quark) distributions in the domain $0.01<x<0.5$. A detailed discussion of jets and comparison of data and theory at the Tevatron can be seen in [278].

Very soon, similar but more sensitive analyses will also be undertaken at the LHC and a beginning has already been made. In Fig. 43 (right-hand frame), we show a comparison between the measured $p_{T}$ spectra by the CMS collaboration [274] at the LHC with $\sqrt{s}=7 \mathrm{TeV}$ and an integrated luminosity of $60 n b^{-1}$ for the calorimeter jets and theory predictions at the next-to-leading (NLO) order accuracy, using an anti- $k_{T}$ jet algorithm with $R=0.5$. Data are divided in several intervals of rapidity $y$ bins. Theory predictions are based on NLOJET ++ 279] with CTEQ-6.6 [231] sets of parton distribution functions (PDF). The non-perturbative (NP) corrections are estimated using two different hadronisation models, PYTHIA 215] and HER$\mathrm{WIG}++$ [280], with the mean of the two predictions taken as the correction. Despite currently modest LHC luminosity, jets having transverse momenta up to $800 \mathrm{GeV}$ are measured and the agreement with QCD is excellent. An in-depth review discussing the physics basis and use of the general purpose Monte Carlo event generators for hadronic collisions at the LHC is available [281, to which we refer for a comprehensive discussion.

Experiments at the LHC have opened a window to sub-energies in the $\mathrm{TeV}$ range for studying jet phenomena in QCD, enabling searches for physics beyond-the-SM in a number of such extensions. Both ATLAS and CMS have searched for new heavy particles, which manifest themselves as narrow resonances in their data collected at the LHC at $\sqrt{s}=7 \mathrm{TeV}$. Such new states may include an excited composite quark $q^{*}$, expected in theories with quark substructure [282 283|284]; an axigluon predicted by chiral colour-models 285|286; a flavour-universal colour-octet coloron [287/288]; or a colour-octet techni- $\rho$ meson predicted by models of extended technicolor and topcolor-assisted tecnicolor 289290291292 .

The dijet invariant mass $\left(m_{j j}\right)$ is an observable which is particularly sensitive to such new objects. This was studied already at the Tevatron in $p \bar{p}$ collisions with negative results, exemplified by the CDF limit on the mass of the excited quarks $q^{*}$ in which a mass range $260<m_{q^{*}}<870 \mathrm{GeV}$ was excluded at $95 \%$ C.L. [293]. ATLAS has extended this exclusion range to higher $q^{*}$ masses, with the range $0.40<m_{q^{*}}<$ $1.26 \mathrm{TeV}$ now excluded using $p p$ collisions 294 . Fig. 44 shows the predicted signal for $q^{*}$ masses of 500, 800, and $1200 \mathrm{GeV}$ satisfying all event selection cuts. No signal of $q^{*}$ is found and the data are in excellent agreement with the background estimates based on the SM.

Similar measurements of the dijet invariant mass spectrum and search for new particles decaying to dijets have been performed by the CMS collaboration [295]. The highest observed dijet mass by CMS at $\sqrt{s}=7 \mathrm{TeV}$ is $2.13 \mathrm{TeV}$. No deviations are found from QCD up to this dijet mass. In particular, string resonances with a mass less than $1.67 \mathrm{TeV}$ have been excluded by the current CMS measurements at 95\% C.L. The sensitivities to the narrow resonances in the dijet mass will increase substantially with the increase in the LHC luminosity and energy. For example, for the anticipated luminosity of $1 \mathrm{fb}^{-1}$ at $\sqrt{s}=7 \mathrm{TeV}$, the expected limits are all in the range of 2.5 to $3.5 \mathrm{TeV}$.

\subsubsection{Physics of the top quark and $\left(W^{ \pm}, Z\right)$ bosons using jets}

Inclusive jet production in $p \bar{p}$ and $p p$ collisions in association with a $Z / \gamma^{*} / W$ boson provides a stringent test of QCD. As these final states are also of great importance in 


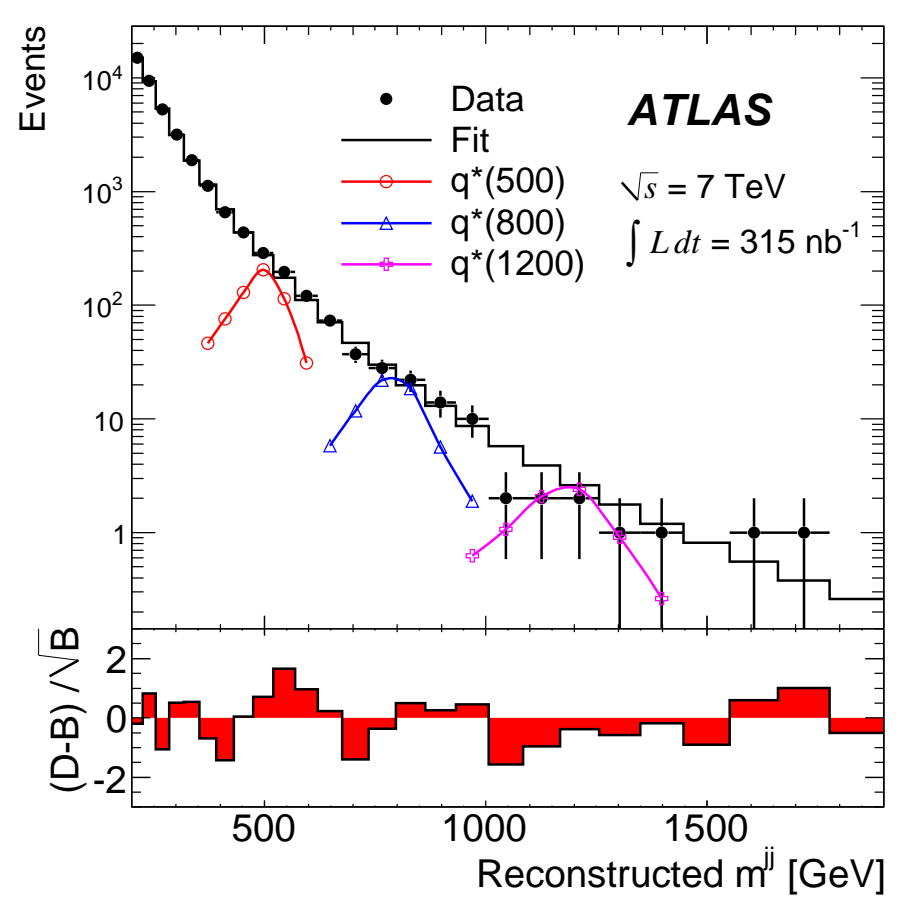

Fig. 44. The data (D) dijet mass distribution (filled points) fitted using a binned background (B) distribution described in the text (histogram). The predicted excited quark $q^{*}$ signals for excited quark masses of 500, 800 and $1200 \mathrm{GeV}$ are overlaid, and the significance of the data-background difference is shown (from ATLAS collaboration [294]).

the search of the SM Higgs boson arising from the process $p p(\bar{p}) \rightarrow W / Z+H(\rightarrow b \bar{b})$, and in the search of supersymmetry in the missing $E_{T}+$ jets channel, the processes $p p(\bar{p}) \rightarrow W / Z / \gamma^{*}+$ jets have received a lot of theoretical and experimental attention. In particular, theoretical predictions for vector boson production recoiling against a hadron jet at next-to-leading order were presented in [296 297 298299]. The processes $p+\bar{p} \rightarrow W / Z / \gamma^{*}+2$ jets to the same level of theoretical accuracy were calculated for the Tevatron in [300] and the corresponding processes $p+p \rightarrow W / Z / \gamma^{*}+2$ jets for the LHC in [301]. Vector boson production in association with $n$-jets for $n \leq 4$ was calculated in 302 303. A parton-level event generator, called MCFM [304], which gives theoretical predictions for a large number of processes containing $W, Z$ and $H$ bosons and jets (including heavy quark jets) is available for the Tevatron and the LHC colliders. Similar theoretical tools have been developed which give predictions for the transverse momentum distributions of the $Z / \gamma^{*} / W$ produced in hadron collisions, based either on fixed order perturbation theory, such as [305] and [306, or based on soft gluon resummations valid at low $p_{T}$ [307], such as RESBOS [308]. They have been used in conjunction with the PDFs [231] in the analysis of the Tevatron data [309 310], and we show below representative measurements from the CDF Collaboration in Figs. 45, The NLO pQCD MCFM framework describes the data rather well over a large range of $p_{T}^{\text {jet }}$, as well as the jet-multiplicity.

The production of heavy gauge boson pairs $(W W, W Z, Z Z)$ in $p \bar{p}$ and $p p$ collisions provides tests of the self-interactions of the gauge bosons and hence deviations from the SM-based predictions for the production rate could indicate new physics [311. Since, topologically diboson production is similar to the associated Higgs boson pro- 

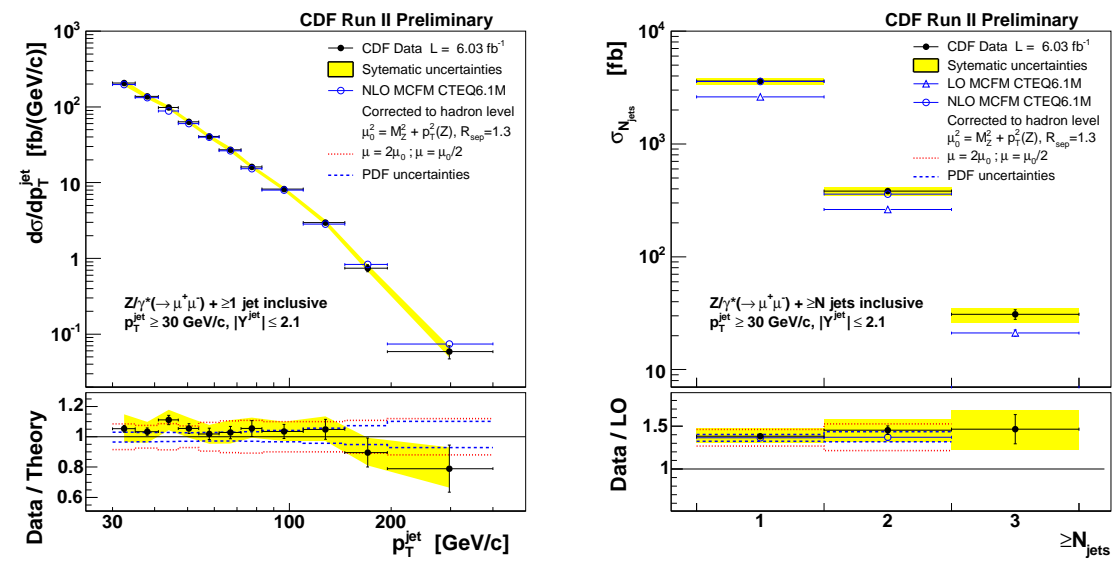

Fig. 45. Left-hand frame: (top) Inclusive jet differential cross section measured by the CDF collaboration as a function of $p_{T}^{\text {jet }}$ in $Z / \gamma^{*}+\geq 1$ jet events (black dots) compared to NLO pQCD predictions (open circles). (bottom) Data/Theory versus $p_{T}^{\text {jet }}$. Right-hand frame: (top) Measured total cross section for inclusive jet production in $Z / \gamma^{*} \rightarrow \mu^{+} \mu^{-}$events as a function of $N_{\text {jet }}$ compared to LO and NLO pQCD predictions. (bottom) Ratio of data and LO pQCD predictions versus $N_{\text {jet }}$. (From 310]).

duction $p p(\bar{p}) \rightarrow V H+X(V=W, Z)$, the experimental techniques developed in $p p(\bar{p}) \rightarrow V V$ are important for the Higgs boson searches as well. The process $p \bar{p} \rightarrow V V$ with both the vector mesons decaying into lepton pairs $\left(W^{ \pm} \rightarrow \ell^{ \pm} \nu_{\ell} ; Z \rightarrow \ell^{+} \ell^{-}\right)$has been observed at the Fermilab Tevatron experiments by CDF [312]313] and D0 [314]. Diboson production has not been conclusively observed in decay channels involving only hadrons. However, evidence for diboson decays into a mixed $\ell \bar{\nu}_{\ell} q \bar{q}$ final state $(\ell=e, \mu, \tau ; q=u, d, s, c, b)$ has been presented by D0 [315] and CDF [316. The experimental analyses involve large transverse momentum imbalance (due to the escaped neutrino) and two jets whose invariant mass can be reconstructed. Because of the limited resolution in the dijet invariant mass, decays of $W^{ \pm} \rightarrow 2$ jets and $Z \rightarrow 2$ jets are not distinguished separately. The most significant backgrounds to the diboson signals are $W(\ell \bar{\nu})+$ jets, $Z(\nu \bar{\nu})+$ jets and QCD multijet production.

In Fig. 466, we show the dijet mass distribution from the $e \nu q \bar{q}$ and $\mu \nu q \bar{q}$ channels for the D0 data 315 and MC predictions. A clear diboson signal in the dijet invariant mass is seen in the lower frame. The resulting cross section $\sigma(W V)=20.2 \pm 4.5 \mathrm{pb}$ is consistent with the SM prediction $\sigma(W V)=16.1 \pm 0.9 \mathrm{pb}$ at $\sqrt{s}=1.96 \mathrm{TeV}[317$. Fig. 47 shows the corresponding measurements by CDF 316. This yields a combined $W W+W Z+Z Z$ cross section in $p \bar{p}$ collisions at $\sqrt{s}=1.96 \mathrm{TeV}: \sigma(p \bar{p} \rightarrow V V)=$ $18.0 \pm 2.8$ (stat) \pm 2.4 (syst) \pm 1.1 (lumi) pb, consistent with the SM prediction.

At the Fermilab Tevatron, top quarks are produced mostly in pairs $p \bar{p} \rightarrow t \bar{t}+X$. In the SM, top quarks decay into a $W$ boson and a $b$ quark almost $100 \%$ of the time. The topology of the final states resulting from the $t \bar{t}$ production depends on whether the $W$ boson decays leptonically $W \rightarrow \ell \nu_{\ell}$, or hadronically $W \rightarrow q \bar{q}^{\prime}$ leading to two jets. Following this, $t \bar{t}$ events have been measured in dilepton $\ell^{+} \ell^{-}+X$, single lepton $\ell^{ \pm}+4$ jets and also in the non-leptonic mode with no energetic leptons. The non-leptonic $t \bar{t}$ final state has the advantage of a large branching ratio $(\simeq 4 / 9)$. The major challenge of this channel is the large background from QCD multijet production. To increase the purity of the candidate sample, methods based on artificial neural networks are applied to the data. Further improvement is then obtained from the requirement of 

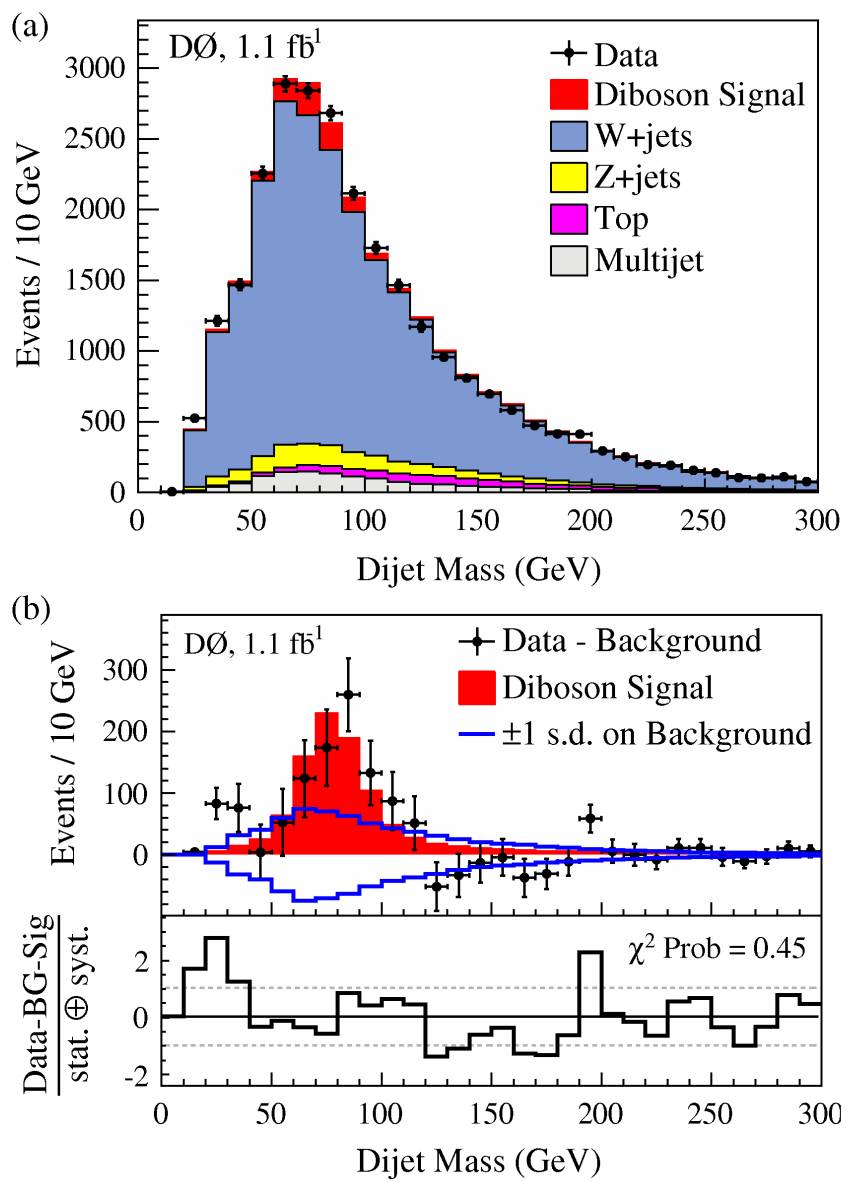

Fig. 46. (a) The dijet mass distribution from the combined $e \nu q \bar{q}$ and $\mu \nu q \bar{q}$ channels for data from the D0 collaboration at the Tevatron and MC predictions. (b) A comparison of the extracted signal (filled histogram) to background-subtracted data (points), along with the $\pm 1 \sigma$ systematic uncertainty on the background. The residual distance between the data points and the extracted signal, divided by the total uncertainty, is shown at the bottom [D0 [315]].

at least one jet identified as originating from a $b$ quark using a secondary vertex $b$ tagging algorithm. These techniques have allowed one to measure the top quark mass and the $t \bar{t}$ cross section in spite of the overwhelming QCD multijet production.

To these ends, a reconstructed top quark mass, $m_{t}^{\text {rec }}$, is determined by fitting the kinematics of the six leading jets from the process $p \bar{p} \rightarrow t \bar{t}+X \rightarrow 6$ jets. There exists a strong correlation between $m_{t}^{\text {rec }}$ and the jet energy scale JES. However, the JES can be calibrated using a selected sample of $t \bar{t}$ candidate events, where a second variable $m_{W}^{\text {rec }}$ is reconstructed from the jets assigned to the $W$ boson. The variable $m_{W}^{\text {rec }}$ is related to the $W^{ \pm}$boson mass, which is known accurately. Relating $m_{t}^{\text {rec }}$ and $m_{W}^{\text {rec }}$ to match the experimental data (in situ calibration) reduces significantly the systematic errors. Further improvement comes by using a multivariate approach taking advantage of the distinctive features of the signal and background events through a neural network. Fig. 48 shows the histogram of $m_{t}^{\text {rec }}$ as obtained in the data and compared to the distributions in the so-called 1-tag and $\geq 2$-tag events from signal and background 


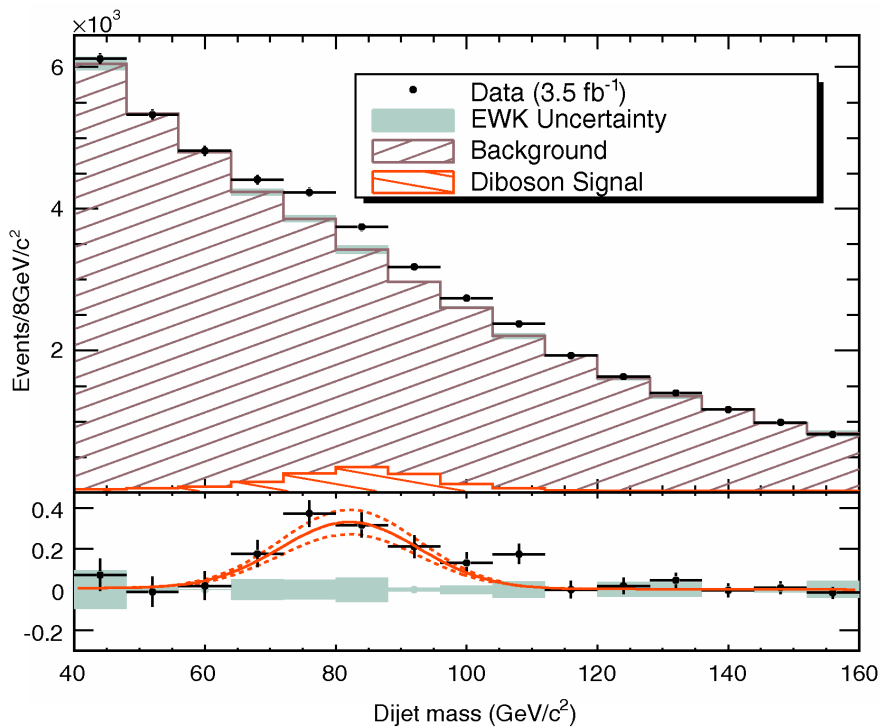

Fig. 47. Top: Comparison between data in $p \bar{p} \rightarrow V V+X)\left(V=W^{ \pm}, Z^{0}\right)$ from the CDF collaboration at the Fermilab Tevatron at $\sqrt{s}=1.96 \mathrm{TeV}$ and the fitted background only in the Dijet invariant mass. Bottom: Comparison of the diboson signal (solid line) with the background subtracted data (points). The dashed lines represent the $\pm 1 \sigma$ statistical variations on the signal [CDF [316]].

corresponding to $M_{\mathrm{top}}=175 \mathrm{GeV}$. The best estimates of the top quark mass from this analysis is 318

$$
M_{\text {top }}=174.8 \pm 2.4(\text { stat }+\mathrm{JES}) \mathrm{GeV} .
$$

The procedure used to measure the top quark mass also returns the average number of signal events expected, given the selected data samples. These results can be turned into a measurement of the $t \bar{t}$ cross section, and yield

$$
\sigma_{t \bar{t}}=7.2 \pm 0.5(\text { stat }) \pm 1.0 \text { (syst) } \pm 0.4(\text { lum }) \mathrm{pb}
$$

\subsubsection{Searches for the Higgs particles}

We have discussed numerous electroweak processes at the Tevatron in which jets play an essential role in the analysis. In particular, $W^{ \pm}$and $Z$ gauge bosons and top quarks have been measured using jets. The last and the most-prized on this list is the Higgs boson. This is being searched for at the Tevatron feverishly. For $m_{H}<135 \mathrm{GeV}$, the dominant decay mode is $H \rightarrow b \bar{b}[319$; analyses of this decay mode open a powerful new Higgs discovery channel [320]. The dominant production modes are $g g \rightarrow H$ and $q \bar{q} \rightarrow H$. The $b \bar{b}$ signal in this channel is overwhelmed by the QCD $b \bar{b}$ production. A promising production and search strategy is the production of a Higgs boson decaying to a pair of bottom quarks in association with a vector boson $V$ ( $W$ or $Z$ ) decaying to quarks or leptons, resulting into a four-jet or a charged lepton + two-jet final states. In either case, two of the jets are required to have secondary vertices consistent with $B$-hadron decays. So far Tevatron Run II searches have used signatures where the $V$ decays to leptons (see, for example Refs. 321322]). Recently, also searches in the 

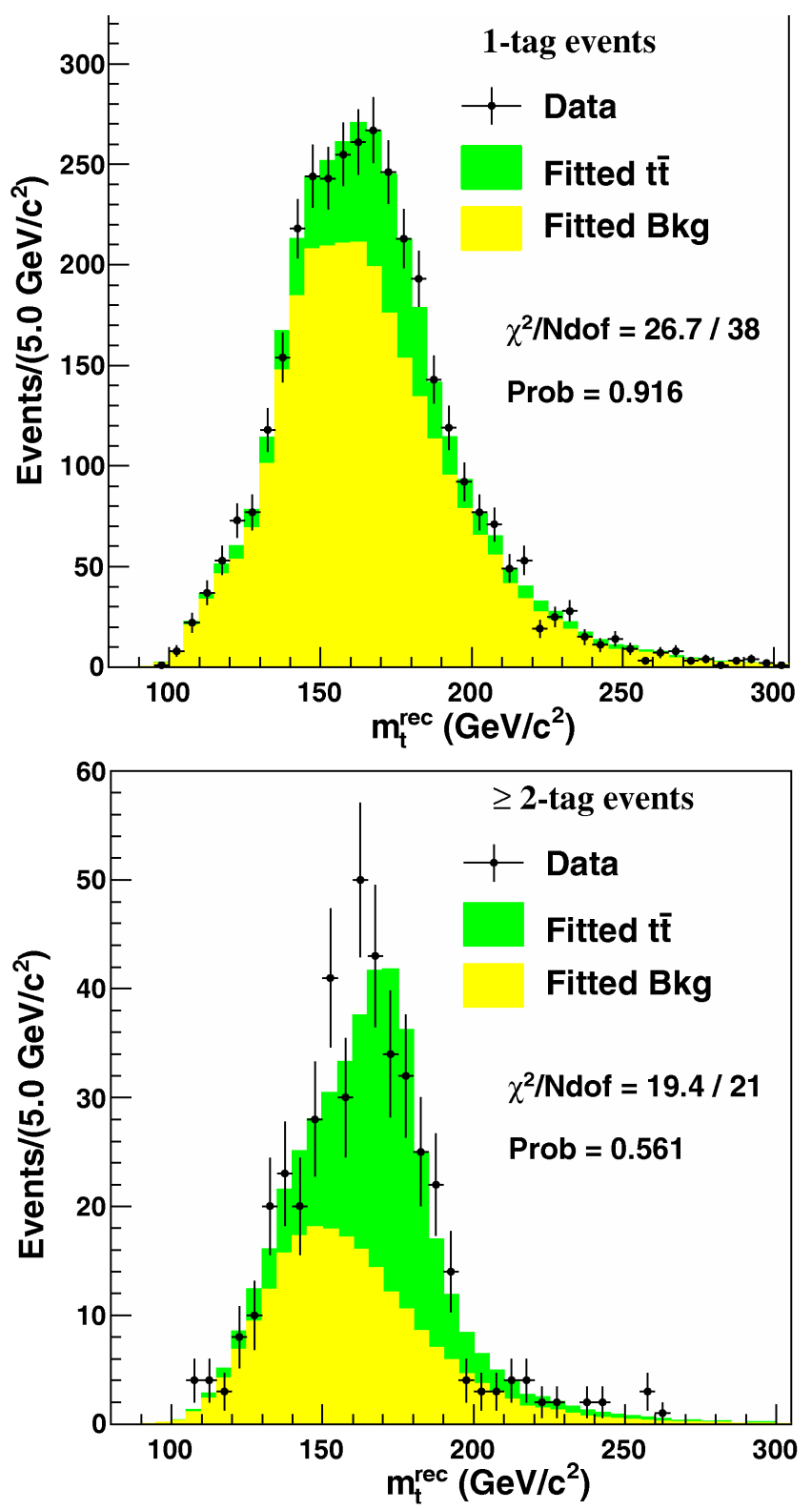

Fig. 48. Histogram of $m_{t}^{\text {rec }}$ from the CDF data (black points) for 1-tag (upper plot) and $\geq 2$ tag events (lower plot) compared to the distributions from signal and background corresponding to $m_{\mathrm{top}}=175 \mathrm{GeV}$. [CDF [318]].

four-jet channels have been reported 323. Using an integrated luminosity of $2 \mathrm{fb}^{-1}$, Higgs boson searches in this channel provide a weak upper bound. For example, for $m_{H}=120 \mathrm{GeV}$, CDF is able to exclude a Higgs production cross section larger than 38 times the SM prediction! Hence, establishing the Higgs signal in this channel requires much more statistics, but also some fundamental progress in jet algorithms to be more efficient in Higgs (and other similar particle) searches. 
New opportunities are offered by observing $b$ jets in Higgs decays at the LHC. The key technique is the 2-jet splitting of a fat $b \bar{b}$ jet generated in events in which the Higgs boson is boosted to large transverse momenta in the processes $p p \rightarrow W^{ \pm} H$ and $Z H$. If the "fat" jet is characterised by a jet radius $R=R_{b b} \simeq M_{H} / p_{T}$, the clustering is partially undone by shrinking the radius $R$ until the fat jet $R_{b \bar{b}}$ decomposes into two slim $R_{b}$ subjets with significantly lower mass, each containing a $b$ quark. Additional criteria will reduce the contamination by standard QCD processes. Though the boost will strongly reduce the event rate, the significance will nevertheless be raised to such a level that light Higgs events can clearly be isolated above background. Extending the method to the channel $p p \rightarrow t \bar{t} H \rightarrow t \bar{t} b \bar{b}$, the crucial $t t H$ coupling, apparently not accessible at LHC otherwise, can be measured in the light Higgs sector 324].

The concept is useful also for the analysis of other processes, for example, the search for supersymmetric particles decaying to jets from the hadronic decays of the electroweak and Higgs bosons [325], or for detecting strongly interacting $W^{ \pm}, Z$ bosons [326], or the search for heavy resonances in decays to top-quark jets [327.

\section{Summary}

Quantum Chromodynamics has been established experimentally in the past four decades as the microscopic theory of the strong interactions, formulated as a nonabelian gauge theory for coloured quarks and gluons within the Standard Model of particle physics. Jet physics has been a crucial instrument for achieving this fundamental result. The beginning was made at SPEAR with the observation of quark jets in $e^{+} e^{-}$annihilation by the SLAC-LBL collaboration 21. Subsequent studies undertaken, in particular at DORIS, PEP and PETRA, involving higher center-of-mass energies largely consolidated the phenomenological profile of the quark jets (see [54] for a review). In fact, jets provide an irrefutable case for the existence of quarks as dynamical entities directly observable in particle detectors, despite colour confinement, convincing even the most die-hard skeptics about the reality of quarks. Moreover, making use of the larger masses of the $b$ - and $c$-quarks, relatively long half-lifes of the corresponding hadrons and their characteristic decay patterns, one can efficiently flavour-tag the heavy quark jets. In the meanwhile, these techniques have been developed to the level of a diagnostic tool to search for new phenomena in which heavy quarks play a role. The decays $t \rightarrow b W$ and $H \rightarrow b \bar{b}$ are two good cases in point.

Theoretically, the existence proof of quark jets in fixed order perturbative QCD was provided by Sterman and Weinberg [45] using a jet-cone definition which coincided with the actual process of detection of hadrons in finite segments of hadron calorimeters. Subsequently, Sterman [46] provided an all-orders argument for the infra-red safety of jet cross sections. Phenomenologically, quark jets follow from the observation that the transverse momenta of the hadrons produced in the fragmentation $q^{*} \rightarrow$ hadrons is limited, whereas the longitudinal components of the hadron momenta scale with energy. A very intuitive and largely accurate quark jet fragmentation model was developed along these lines by Field and Feynman [37, which played an important role in the quantitative analysis of jet data.

Analysis of the decays $\Upsilon(9.46) \rightarrow$ hadrons measured by the PLUTO collaboration 24] working at DORIS were undertaken in terms of the underlying perturbative process $\Upsilon(9.46) \rightarrow g g g$. In particular, the experimental profile of the most energetic parton $\left(\left\langle E_{1}\right\rangle \simeq 4.1 \mathrm{GeV}\right)$ was close to the phenomenological expectations of a hadron jet. However, a clear three-jet topology using en vogue jet definitions was not established in $\Upsilon(9.46)$ decays for lack of energy [24]. This three-jet topology was established later in the $e^{+} e^{-}$experiments operating at higher energies (typically $\sqrt{s}=30 \mathrm{GeV}$ ), resulting from the energetic quark, anti-quark and gluon from the 
process $e^{+} e^{-} \rightarrow q \bar{q} g$. The jet profile of the three jets as well as the inclusive hadronic measurements undertaken at PETRA in 1979 followed detailed theoretical expectations 303132 . Thus, it is fair to conclude that the study of the decay $\Upsilon(9.46) \rightarrow 3 g$, initiated by PLUTO at DORIS [24, was an important step in the confirmation of QCD which served as a prelude to the unambiguous discovery of the three-jet topology by the experiments at PETRA. More detailed and quantitative tests of perturbative QCD in the decays of $\Upsilon(9.46)$ were also presented subsequently by PLUTO [11]. Theoretical proofs of the existence of three-jet topologies, in the Sterman-Weinberg sense, were provided in 1981, and somewhat later in terms of the next-to-leading order calculations of the three-jet cross sections. This was done for inclusive jet distributions (such as the Fox-Wolfram shape variable [142 143], thrust 144145] and energy-energy correlations [153 154 157 158]) and in terms of topological jet cross sections [163 164 165166 167 169]. Confirmation of the non-abelian character of QCD in jets 218 219220221 in the four-parton processes $e^{+} e^{-} \rightarrow q \bar{q} g g$ came from experiments at LEP [222 223]. In the meanwhile, multijet physics has developed enormously, with the NLO calculation of $e^{+} e^{-} \rightarrow \gamma, Z \rightarrow 4$ jets 210211212 completed around 1996, and the NLO calculation to five-jet production at LEP reported recently [214.

The properties of gluon jets have largely been determined by experiments at PETRA and subsequently at LEP. The fragmentation of gluon jets was initially conceived by treating them as independent partons [31], or implemented by the perturbative process $g^{*} \rightarrow q \bar{q}$ as the first step followed by incoherent quark fragmentation [32] (IJ models). The resulting picture could largely account for the essential properties of the two- and three-jet events seen at PETRA and PEP, and they helped in the discovery of three-jet events (and hence, of gluons) at PETRA. However, analysis of the PETRA jets saw the emergence of an alternative fragmentation scheme for the $e^{+} e^{-} \rightarrow q \bar{q} g$ events - the LUND string model [38 - in which hadronisation was implemented in terms of two strings stretched along the colour-anticolour axes which then fragmented very much like the quark-antiquark string in $e^{+} e^{-} \rightarrow 2$-jets. This model provided a better phenomenological description of data, in particular the particle flow between the quark, antiquark and gluon jets 179 180181182. The LUND-string effect was subsequently understood in perturbation theory in terms of the antenna radiation pattern of QCD [48, reflecting the colour coherence effect of the non-abelian character of this theory. Detailed fragmentation models were built along the angle-ordered perturbation theory, which preserve the colour coherence in QCD, and in which parton showers were included in the form of cascades. which then finally fragmented as hadron clusters according to phase space (cluster hadronisation models) [39]. These Monte Carlo models developed for the PETRA jet analysis have played an important role in the analysis of all high energy data involving jets. The modern incarnation of these fragmentation models are PYTHIA [49], HERWIG [51] and SHERPA [52], which differ in details on how the parton showers are matched on to the fixed order perturbative QCD matrix elements and in the hadronisation schemes. A central role is also played by the jet algorithms, which starting from the JADE scheme 44 have now evolved as trustworthy tools in the definition of jets, with the modern versions called the $k_{T}$ [78. (mostly in $e^{+} e^{-}$annihilation processes) and anti- $k_{T}$ jet algorithms [82.

Another large application of QCD is in studying DIS, photoproduction and $\gamma \gamma$ collisions. In these cases, initial states are not so well known as in $e^{+} e^{-}$annihilation. Jets and QCD have played a central role in mapping the PDFs of the proton and the photon. We have summarised some of the highlights in this article. In particular, DIS measurements at HERA [235/236 237/238|239|240] have firmly established the rise of the structure function $F_{2}\left(x, Q^{2}\right)$, which is due to the rapid growth of the gluon density $g\left(x, Q^{2}\right)$ for low values of $x$ as $Q^{2}$ increases. Likewise, high energy $p \bar{p}$ collisions at the Tevatron, in particular the Tevatron Run II data on inclusive jet production 273 276 277, have led to greatly firming up the PDFs of the proton. On the 
theoretical side, the complete next-to-next-to leading order (3-loop) parton splitting functions have been derived by Moch et al. 328 329. They have been used in working out the proton PDFs by the CTEQ 231 and the MSTW 232 collaborations. Thus, the HERA and the Tevatron measurements and the progress in the QCD splitting functions will prove to be an asset in understanding the forthcoming LHC data.

In the meanwhile, a fundamental change of paradigm has taken place concerning "Jets and QCD". The theory (QCD) is so well controlled (in particular, $\alpha_{s}\left(M_{Z}\right)$ is known to an accuracy of better than $1 \%$ and the crucial property of asymptotic freedom is now fully established) that jet-physics can serve as a tool to chart out new territories in high energy physics. We have reviewed here some applications of jet techniques in quantifying the properties of the top quark and the electroweak gauge bosons $(W, Z)$. They have already played a significant role in determining the properties of the SM particles in experiments at the Tevatron and they will play an even more important role in the analysis of data from the experiments at the LHC. For example, jets are now increasingly used in developing search strategies for the Higgs boson, and even particles suggested in theories beyond the Standard Model. New jet techniques, such as the 2 -jet splitting of a fat $b \bar{b}$ jet will be required to disentangle the decay $H \rightarrow b \bar{b}$ from an overwhelming QCD background in hadron colliders.

One problem in jet physics however remains unsolved up to now. While, due to asymptotic freedom, the dynamics of quarks and gluons can theoretically be described with high accuracy at short distances, matched by numerical lattice calculations for static properties of hadrons, the transition from small to large distances in the evolution of jets is theoretically not understood. However, a bridge is built, at the present time, by intuitively formulated models, which are constrained experimentally so stringently that hadron jets can be exploited to draw a valid picture of quarks and gluons and their interactions at short distances. New theoretical methods may help solve this multi-scale problem rigorously in the future.

Acknowledgements We thank Peter Zerwas for the collaboration in the early stages of this work, for numerous helpful discussions that we had with him all along, and for his valuable input in good parts of this manuscript. Helpful discussions with Hans-Jürgen Meyer, Hinrich Meyer and Bruno Stella on the PLUTO analysis of the $\Upsilon$ (9.46) data are thankfully acknowledged. We also thank a large number of our colleagues and collaborators whose dedicated and painstaking work over decades has contributed decisively to the development of QCD and jet physics. This article is dedicated collectively to all of them.

\section{References}

1. H. Fritzsch and M. Gell-Mann, Current Algebra: Quarks and What Else?, Proceedings, 16th Int. Conference on High Energy Physics, Batavia IL, eConf C720906V2: 135-165 (1972)

2. H. Fritzsch, M. Gell-Mann and H. Leutwyler, Advantages of the Color Octet Gluon Picture, Phys. Lett. B 47,365 (1973)

3. D. J. Gross and F. Wilczek, Ultraviolet Behavior of Non-Abelien Gauge Theories, Phys. Rev. Lett. 30, 1343 (1973)

4. H. D. Politzer, Reliable Perturbative Results for Strong Interactions?, Phys. Rev. Lett. 30, 1346 (1973)

5. M. Gell-Mann, A Schematic Model of Baryons and Mesons, Phys. Lett. 8, 214 (1964)

6. G. Zweig, An SU(3) Model for Strong Interaction Symmetry and its Breaking, CERNTH-401 (1964)

7. K. Nakamura et al., [Particle Data Group], Review of Particle Physics, J. Phys. G 37, $075021(2010)$ 
8. O. W. Greenberg, Spin and Unitary Spin Independence in a Paraquark Model of Baryons and Mesons, Phys. Rev. Lett. 13, 598 (1964)

9. M. Y. Han and Y. Nambu, Three-triplet Model with Double SU(3) Symmetry, Phys. Rev. 139, B1006 (1965)

10. M. Gell-Mann, Quarks, Acta Phys. Austr. Suppl. IX, 733 (1972)

11. Y. Nambu, A Systematics of Hadrons in Subnuclear Physics, in Preludes in theoretical physics, North-Holland Publishing Company, Amsterdam (1966)

12. C. N. Yang and R. L. Mills, Conservation of Isotopic Spin and Isotopic Gauge Invariance, Phys. Rev. 96, 191 (1954)

13. K. G. Wilson, Confinement of Quarks, Phys. Rev. D 10, 2445 (1974)

14. C. H. Llewellyn Smith, Inelastic Lepton Scattering in Gluon Models, Phys. Rev. D 4, $2392(1971)$

15. D. J. Gross, How to Test Scaling in Asymptotically Free Theories, Phys. Rev. Lett. 32, $1071(1974)$

16. R. P. Feynman, The Behavior of Hadron Collisions at Extreme Energies, in Proceedings of the 3rd Topical Conference on High Energy Collision of Hadrons, Stony Brook, N. Y., 237 (1969)

17. R. M. Barnett et al. [Particle Data Group], Review of particle physics. Particle Data Group, Phys. Rev. D 54, 1 (1996)

18. N. Cabibbo, G. Parisi and M. Testa, Hadron Production in $e^{+} e^{-}$Collisions, Lett. Nuovo Cim. 4S1, 35 (1970) [Lett. Nuovo Cim. 4, 35 (1970)]

19. S. D. Drell, D. J. Levy and T. M. Yan, Theory of Deep-Inelastic Lepton-Nucleon Scattering and Lepton-Pair Annihilation Processes, iii: Deep-Inelastic Electron-Positron Annihilation, Phys. Rev. D 1, 1617 (1970)

20. S. M. Berman, J. D. Bjorken and J. B. Kogut, Inclusive Processes at High Transverse Momentum, Phys. Rev. D 4, 3388 (1971)

21. G. Hanson et al. [SLAC-LBL Collaboration], Evidence for Jet Structure in Hadron Production by $e^{+} e^{-}$Annihilation, Phys. Rev. Lett. 35, 1609 (1975)

22. G. Hanson et al. [SLAC-LBL Collaboration], Hadron Production by $e^{+} e^{-}$Annihilation at Center-of-Mass Energies between $2.6 \mathrm{GeV}$ and $7.8 \mathrm{GeV}$, Part 2: Jet Structure and Related Inclusive Distributions, Phys. Rev. D 26, 991 (1982)

23. J. D. Bjorken and S. J. Brodsky, Statistical Model for Electron-Positron Annihilation into Hadrons, Phys. Rev. D 1, 1416 (1970)

24. Ch. Berger et al. [PLUTO Collaboration], Jet Analysis of the Upsilon(9.46) Decay into Charged Hadrons, Phys. Lett. B 82, 449 (1979)

25. R. Brandelik et al. [TASSO Collaboration], Evidence for Planar Events in $e^{+} e^{-}$Annihilation at High Energies, Phys. Lett. B 86, 243 (1979)

26. D. P. Barber et al. [Mark-J Collaboration], Discovery of Three Jet Events and a Test of Quantum Chromodynamics at Petra Energies, Phys. Rev. Lett. 43, 830 (1979)

27. C. Berger et al. [PLUTO Collaboration], Evidence for Gluon Bremsstrahlung in $e^{+} e^{-}$ Annihilations at High-Energies, Phys. Lett. B 86, 418 (1979)

28. W. Bartel et al. [JADE Collaboration], Observation of Planar Three Jet Events in $e^{+} e^{-}$ Annihilation and Evidence for Gluon Bremsstrahlung, Phys. Lett. B 91, 142 (1980)

29. B. H. Wiik, First Results From Petra, in *Bergen 1979, Proceedings, Neutrino '79, Vol.1*, p. 113

30. J. R. Ellis, M. K. Gaillard and G. G. Ross, Search for Gluons in $e^{+} e^{-}$Annihilation, Nucl. Phys. B 111,253 (1976) [Erratum-ibid. B 130, 516 (1977)]

31. P. Hoyer, P. Osland, H. G. Sander, T. F. Walsh and P. M. Zerwas, Quantum Chromodynamics and Jets in $e^{+} e^{-}$, Nucl. Phys. B 161, 349 (1979)

32. A. Ali, E. Pietarinen, G. Kramer and J. Willrodt, A QCD Analysis of the High-Energy $e^{+} e^{-}$Data from Petra, Phys. Lett. B 93, 155 (1980)

33. M. L. Mangano and T. J. Stelzer, Tools for the simulation of hard hadronic collisions, Ann. Rev. Nucl. Part. Sci. 55, 555 (2005)

34. S. Bethke, QCD studies at LEP, Phys. Rept. 403-404, 203 (2004)

35. A. Breakstone et al. [Ames-Bologna-CERN-Dortmund-Heidelberg-Warsaw Collaboration], High $P_{T}$ Hadrons as Leading Particles in Jets Produced at the ISR. 1. Momentum Distribution of Secondaries in the Trigger Jet, Z. Phys. C 23, 9 (1984) 
36. W. G. Scott [UA1 Collaboration], Jets in the UA1 Experiment, In *Berne 1984, Proceedings, Proton Antiproton Collider Physics*, p. 6

37. R. D. Field and R. P. Feynman, A Parametrization of the Properties of Quark Jets, Nucl. Phys. B 136, 1 (1978)

38. B. Andersson, G. Gustafson and T. Sjostrand, A Three-Dimensional Model for Quark and Gluon Jets, Z. Phys. C 6, 235 (1980)

39. G. Marchesini and B. R. Webber, Simulation of QCD Jets including Soft Gluon Interference, Nucl. Phys. B 238, 1 (1984)

40. S. L. Wu and G. Zobernig, A Method of Three Jet Analysis in $e^{+} e^{-}$Annihilation, Z. Phys. C 2, 107 (1979)

41. K. Lanius, Cluster Methods of Jet Analysis, DESY Report 80/36 (1980)

42. K. Lanius, H. E. Roloff and H. Schiller, Selection of Jets in Multi - Hadron Final States Produced by $e^{+} e^{-}$Annihilation, Z. Phys. C 8, 251 (1981)

43. H. J. Daum, H. Meyer and J. Bürger, A Cluster Algorithm for Jet Studies, Z. Phys. C 8, $167(1981)$

44. S. Bethke et al. [JADE Collaboration], Experimental Investigation of the Energy Dependence of the Strong Coupling Strength, Phys. Lett. B 213, 235 (1988)

45. G. Sterman and S. Weinberg, Jets from Quantum Chromodynamics, Phys. Rev. Lett. 39, 1436 (1977)

46. G. F. Sterman, Phys. Rev. D19, 3135 (1979)

47. B. Andersson, G. Gustafson, G. Ingelman and T. Sjostrand, Parton Fragmentation and String Dynamics, Phys. Rept. 97, 31 (1983)

48. Y. I. Azimov, Y. L. Dokshitzer, V. A. Khoze and S. I. Troian, The String Effect and QCD Coherence, Phys. Lett. B 165, 147 (1985)

49. T. Sjostrand, PYTHIA 8 Status Report, arXiv:0809.0303 [hep-ph]

50. T. Sjostrand, S. Mrenna and P. Z. Skands, A Brief Introduction to PYTHIA 8.1, Comput. Phys. Commun. 178, 852 (2008)

51. M. Bähr et al., Herwig++ Status Report, Eur. Phys. J., C58 (2008) 639, arXiv:0809.2002 [hep-ph] (2008)

52. T. Gleisberg, S. Hoche, F. Krauss, M. Schönherr, S. Schumann, F. Siegert and J. Winter, Event Generation with SHERPA 1.1, JHEP 0902, 007 (2009)

53. G. Kramer, Theory of Jets in Electron-Positron Annihilation, Springer Tracts in Modern Physics, Nr. 102 (1984)

54. A. Ali and P. Söding, High Energy Electron-Positron Physics, World Scientific (1988)

55. R. K. Ellis, W. J. Stirling and B. R. Webber, $Q C D$ and Collider Physics, Cambridge University Press (1996)

56. G. S. Bali, H. Neff, T. Duessel, T. Lippert and K. Schilling [SESAM Collaboration], Observation of String Breaking in QCD, Phys. Rev. D 71, 114513 (2005)

57. G. Abbiendi et al. [OPAL Collaboration], Leading particle production in light flavor jets, Eur. Phys. J. C 16, 407 (2000) arXiv:hep-ex/0001054

58. S. Albino, B. A. Kniehl and G. Kramer, Fragmentation functions for light charged hadrons with complete quark flavor separation, Nucl. Phys. B 725, 181 (2005) arXiv:hep-ph/0502188.

59. D. H. Saxon, in Ref. 54.

60. S. Albino, The Hadronization of Partons, Rev. Mod. Phys. 82, 2489 (2010)

61. Ya. I. Azimov, L. L. Frankfurt and V. A. Khoze, On Reaction $e^{+} e^{-} \rightarrow$ Hadrons, New Particles and $e^{+} e^{-}$Annihilation (in Russian), Leningrad 76-222 (1976)

62. J. D. Bjorken, Properties of Hadron Distributions in Reactions Containing Very Heavy Quarks, Phys. Rev. D 17, 171 (1978)

63. C. Peterson, D. Schlatter, I. Schmitt and P. M. Zerwas, Scaling Violations in Inclusive $e^{+} e^{-}$Annihilation Spectra, Phys. Rev. D 27, 105 (1983)

64. B. Mele and P. Nason, The Fragmentation function for heavy quarks in QCD, Nucl. Phys. B 361, 626 (1991).

65. J. P. Ma, Perturbative prediction for parton fragmentation into heavy hadron, Nucl. Phys. B 506, 329 (1997) arXiv:hep-ph/9705446 
66. P. Nason and C. Oleari, A Phenomenological Study of Heavy-Quark Fragmentation Functions in $e^{+} e^{-}$Annihilation, Nucl. Phys. B 565, 245 (2000)

67. M. Cacciari, P. Nason and C. Oleari, A Study of Heavy Flavoured Meson Fragmentation Functions in $e^{+} e^{-}$Annihilation, JHEP 0604, 006 (2006)

68. T. Kneesch, B. A. Kniehl, G. Kramer and I. Schienbein, Charmed-meson fragmentation functions with finite-mass corrections, Nucl. Phys. B 799, 34 (2008) arXiv:0712.0481 [hep$\mathrm{ph}]]$

69. B. A. Kniehl, G. Kramer, I. Schienbein and H. Spiesberger, Finite-mass effects on inclusive $B$ meson hadroproduction, Phys. Rev. D 77, 014011 (2008) arXiv:0705.4392 [hep-ph]]

70. D. Amati and G. Veneziano, Preconfinement as a Property of Perturbative QCD, Phys. Lett. B 83, 87 (1979)

71. V. V. Sudakov, Vertex Parts at Very High-Energies in Quantum Electrodynamics, Sov. Phys. JETP 3, 65 (1956) [Zh. Eksp. Teor. Fiz. 30, 87 (1956)]

72. G. Alexander Proceedings of the XIXth International Conference On High Energy Physics (Tokyo, 1978) p. 255

73. S. Brandt, C. Peyrou, R. Sosnowski and A. Wroblewski, The Principal Axis of Jets: An Attempt to Analyze High-energy Collisions as Two-body Processes, Phys. Lett. 12, 57 (1964)

74. E. Fahri, A QCD Test for Jets, Phys. Rev. Lett. 39, 1587 (1977)

75. S. Brandt and H. J. Dahmen, Axes and Scalar Measures of Two-Jet and Three-Jet Events, Z. Phys. C1, 61 (1979)

76. O. Nachtmann and A. Reiter, A New Quantity for Finding Four-Jet Events in ElectronPositron Annihilation into Hadrons, Z. Phys. C 14, 47 (1982)

77. G. P. Salam, Towards Jetography, Eur. Phys. J. C 67, 637 (2010)

78. S. Catani, Y. L. Dokshitzer, M. Olsson, G. Turnock and B. R. Webber, New Clustering Algorithm for Multi - Jet Cross-sections in $e^{+} e^{-}$Annihilation, Phys. Lett. B 269, 432 (1991)

79. S.D. Ellis and D.E. Soper, Successive Combination Jet Algorithm for Hadron Collisions, Phys. Rev. D 48, 3160 (1993)

80. S. Catani et al., Longitudinally Invariant $k_{t}$ Clustering Algorithms for Hadron Hadron Collisions, Nucl. Phys. B 406, 187 (1993)

81. M. Wobisch and T. Wengler, Hadronization Corrections to Jet Cross Sections in DeepInelastic Scattering, arXiv:hep-ph/9907280 (1999)

82. M. Cacciari, G. P. Salam and G. Soyez, The Anti- $k_{t}$ Jet Clustering Algorithm, JHEP 0804, 063 (2008)

83. G. C. Blazey et al., Run II Jet Physics, arXiv:hep-ex/0005012 (2000)

84. J. E. Huth et al., Toward a Standardization of Jet Definitions, FERMILAB-CONF-90249-E (1990), Published in Snowmass Summer Study 1990:0134-136 (QCD161:D15:1990)

85. M. H. Seymour, Jet shapes in hadron collisions: Higher orders, resummation and hadronization, Nucl. Phys. B 513, 269 (1998) arXiv:hep-ph/9707338

86. R. P. Feynman, Photon-Hadron-Interactions (Benjamin, Reading) (1972)

87. R. Gatto, Proceedings of the International Symposium on Electron and Photon Interactions at High Energies (Deutsche Physikalische Gesellschaft e. V., Hamburg 1965) edited by G. Höhler, G. Kramer and U. Meyer-Berkhout, Vol. 1,106 (1965)

88. Ch. Berger et al. [PLUTO Collaboration], A Study of Jets in Electron Positron Annihilations into Hadrons in the Energy Range $3.1 \mathrm{GeV}$ to $9.5 \mathrm{GeV}$, Phys. Lett. B 78, 176 (1978)

89. E. Elsen, Multi-Hadron Production in $e^{+} e^{-}$Annihilation at Petra Energies and Comparison with Quantum Chromodynamics (in German), Interner Bericht DESY F22-81/02 (1981)

90. C. G. Callan, D. J. Gross, High-Energy Electroproduction and the Constitution of the Electric Current, Phys. Rev. Lett. 22, 156 (1969)

91. T. D. Lee and M. Nauenberg, Degenerate Systems and Mass Singularities, Phys. Rev. 133, B1549 (1964)

92. P. M. Stevenson, Comments on Sterman-Weinberg Jet Formula, Phys. Lett. B 78, 451 (1978) 
93. G. Kramer and B. Lampe, Two-Jet Cross-Section in $e^{+} e^{-}$Annihilation, Z. Phys. C 34, 497 (1987) [Erratum-ibid. C 42, 504 (1989)]

94. W. Bartel et al. [JADE Collaboration], Experimental Studies on Multi-Jet Production in $e^{+} e^{-}$Annihilation at PETRA Energies, Z. Phys. C 33, 23 (1986)

95. S. W. Herb et al., Observation of a Dimuon Resonance at $9.5 \mathrm{GeV}$ in $400 \mathrm{GeV}$ ProtonNucleus Collisions, Phys. Rev. Lett. 39 (1979) 252

96. W. R. Innes et al., Observation of Structure in the Upsilon Region, Phys. Rev. Lett. 39, 1240 (1979)

97. Ch. Berger et al. [PLUTO Collaboration], Observation of a Narrow Resonance in $e^{+} e^{-}$ Annihilation at $9.46 \mathrm{GeV}$, Phys. Lett. B 76, 243 (1978)

98. Ch. Berger et al. [PLUTO Collaboration], Determination of the Electronic Branching Ratio of the Upsilon(9.46) and an Upper Limit for its Total Width, Phys. Lett. B 93, 497 (1980)

99. C. W. Darden et al., Observation of a Narrow Resonance at $9.46 \mathrm{GeV}$ in ElectronPositron Annihilation, Phys. Lett. B 76, 246 (1978)

100. J. K. Bienlein et al., Observation of a Narrow Resonance at $10.02 \mathrm{GeV}$ in $e^{+} e^{-}$Annihilations, Phys. Lett. B 78, 360 (1978)

101. D. Andrews et al. [CLEO Collaboration], Observation of Three Upsilon States, Phys. Rev. Lett. 44, 1108 (1980)

102. T. Böhringer et al., Observation of Upsilon, Upsilon' and Upsilon" at the Cornell Storage Ring, Phys. Rev. Lett. 44, 1111 (1980)

103. T. Appelquist and H. D. Politzer, Orthocharmonium and $e^{+} e^{-}$Annihilation, Phys. Rev. Lett. 34, 43 (1975)

104. T. Appelquist and H. D. Politzer, Heavy Quarks and Long-lived Hadrons, Phys. Rev. D 12, 1404 (1975)

105. K. Koller and T. F. Walsh, Three Gluon Jets as a Test of QCD, Phys. Lett. B 72, 227 (1977)

106. K. Koller and T. F. Walsh, Gluons in Quarkonium Decay, Nucl. Phys. B 140, 449 (1978)

107. K. Koller, H. Krasemann and T. F. Walsh, The 3 Gluon Decay of Quarkonium, Z. Phys. C 1, 71 (1979)

108. T. A. DeGrand, Y. G. Ng. and S. H. H. Tye, Jet Structure in $e^{+} e^{-}$Annihilation as a Test of QCD and the Quark-Confining String, Phys. Rev. D 16, 3251 (1977)

109. A. de Rujula, J. Ellis, E. G. Floratos and M. K. Gaillard, QCD Predictions for Hadronic Final States in $e^{+} e^{-}$Annihilation, Nucl. Phys. B 138, 387 (1978)

110. A. Ore and J. L. Powell, Three Photon Annihilation of an Electron-Positron Pair, Phys. Rev. 75, 1696 (1949)

111. Ch. Berger et al. [PLUTO Collaboration], Topology of the Upsilon Decay, Z. Phys. C8, $101(1981)$

112. B. R. Stella and H. J. Meyer, $\Upsilon(9.46 \mathrm{GeV})$ and the Gluon Discovery (A critical recollection of PLUTO results), Eur. Phys. J. H36, 203 (2011)

113. K. Koller and H. Krasemann, Excluding Scalar Gluons, Phys. Lett. B 88, 119 (1979)

114. T. F. Walsh and P. M. Zerwas, Upsilon(9.46) Decays Do Test QCD, Phys. Lett. B 93, $53(1980)$

115. B. Niczyporuk et al. [LENA Collaboration], Charged Hadron Production in $e^{+} e^{-}$Annihilation in the Upsilon and Upsilon' Region, Z. Phys. C 9, 1 (1981)

116. K. Berkelman, Upsilon Spectroscopy at CESR, Phys. Rep. 98, 145 (1983)

117. H. Albrecht et al. [ARGUS Collaboration], An Upper Limit for Two-Jet Production in Direct Upsilon(1S) Decays, Z. Phys. C 31, 181 (1986)

118. G. Gidal, B. Armstrong and A. Rittenberg, "Major Detectors in Elementary Particle Physics," LBL 91-Supplement (March 1983; updated May 1985)

119. H.L. Lynch, "Detectors for High Energy $e^{+} e^{-}$Physics", in Ref. 54

120. H. Georgi and D. V. Nanopoulos, $t$-Quark Mass in a Superunified Theory, Phys. Lett.

B 82, 392 (1979)

121. H. Fritzsch, Quark Masses and Flavor Mixing, Nucl. Phys. B 155, 189 (1979) 
122. R. Brandelik et al. [TASSO Collaboration], $e^{+} e^{-}$Annihilation at High-Energies and Search for the $t$-Quark Continuum Contribution, Z. Phys. C 4, 87 (1980)

123. C. Berger et al. [PLUTO Collaboration], Search for a 'Top' Threshold in Hadronic $e^{+} e^{-}$ Annihilation at Energies between $22 \mathrm{GeV}$ and $31.6 \mathrm{GeV}$, Phys. Lett. B 86, 413 (1979)

124. N. Cabibbo, Unitary Symmetry and Leptonic Decays, Phys. Rev. Lett. 10, 531 (1963)

125. M. Kobayashi and T. Maskawa, CP Violation in the Renormalizable Theory of Weak Interaction, Prog. Theor. Phys. 49, 652 (1973)

126. [Tevatron Electroweak Working Group and CDF and D0 Collaboration], Combination of CDF and D0 Results on the Mass of the Top Quark, FERMILAB-TM-2427E, TEVEWWG-TOP-2009-03, CDF-NOTE-9717, D0-NOTE-5899, arXiv:0903.2503 [hepex]] (2009)

127. A. Ali, J. G. Körner, J. Willrodt and G. Kramer, Indications of $b \bar{b}$ Production and Decay at Petra, Phys. Lett. B 83, 375 (1979)

128. C. Berger et al. [PLUTO Collaboration], Cross-Sections and Event Topologies in $e^{+} e^{-}$ Annihilation at $13 \mathrm{GeV}$ and $17 \mathrm{GeV}$ Observed with the Pluto Detector, Phys. Lett. B 81, 410 (1979)

129. R. Brandelik et al. [TASSO Collaboration], Properties of Hadron final States in $e^{+} e^{-}$ Annihilation at $13 \mathrm{GeV}$ and $17 \mathrm{GeV}$ Center-of-Mass Energies, Phys. Lett. B 83, 261 (1979)

130. A. Ali, J. G. Körner, G. Kramer and J. Willrodt, Jet-Like Distributions from the Weak Decay of Heavy Quarks, Z. Phys. C 1, 203 (1979)

131. A. Ali, J. G. Körner, G. Kramer and J. Willrodt, Nonleptonic Weak Decays of Bottom Mesons, Z. Phys. C 1, 269 (1979)

132. H. Schopper, First Results from PETRA Provide New Aspects of the Basic Structure of Matter, Naturwiss. 67, 161 (1980)

133. S. L. Wu, Hadron Jets and the Discovery of the Gluon, in the Proceedings of 3rd International Symposium on the History of Particle Physics: The Rise of the Standard Model, Stanford, California, 24-27 Jun 1992, p. 600

134. J. G. Branson, Gluon Jets, Talk given at International Conference on the History of Original Ideas and Basic Discoveries in Particle Physics, Erice, Italy, 29 Jul - 4 Aug (1994)

135. P. Söding, B. Wiik, G. Wolf and S. L. Wu, The First Evidence for Three-Jet Events in $e^{+} e^{-}$Collisions at PETRA: First Direct Observation of the Gluon, Talk given at Award Ceremony of the 1995 EPS High Energy and Particle Physics Prize. Brussels, Belgium, 27 Jul - 2 Aug 1995, Report DESY-96-193 (1996) Published in Brussels EPS HEP 1995:3-14 (QCD161:I48:1995)

136. J. Ellis, Those were the Days: Discovering the Gluon, CERN Cour. 49N6, 15 (2009)

137. P. Söding, On the Discovery of the Gluon, Eur. Phys. J. H 35, 3 (2010)

138. G. C. Fox and S. Wolfram, Observables for the Analysis of Event Shapes in $e^{+} e^{-}$ Annihilation and Other Processes, Phys. Rev. Lett. 41, 1581 (1978)

139. J. R. Ellis and I. Karliner, Measuring the Spin of the Gluon in $e^{+} e^{-}$Annihilation, Nucl. Phys. B 148, 141 (1979)

140. A. Ali, J. G. Körner, Z. Kunszt, J. Willrodt, G. Kramer, G. Schierholz and E. Pietarinen, Four-Jet Production in $e^{+} e^{-}$Annihilation, Phys. Lett. B 82, 285 (1979)

141. QCD Predictions for Four-Jet Final States in $e^{+} e^{-}$Annihilation, Nucl. Phys. B 167, 454 (1980)

142. R. K. Ellis, D. A. Ross and A. E. Terrano, Calculation of Event Shape Parameters in $e^{+} e^{-}$Annihilation, Phys. Rev. Lett. 45, 1226 (1980)

143. R. K. Ellis, D. A. Ross and A. E. Terrano, The Perturbative Calculation of Jet Structure in $e^{+} e^{-}$Annihilation, Nucl. Phys. B 178, 421 (1981)

144. J. A. M. Vermaseren, K. J. F. Gaemers and S. J. Oldham, Perturbative QCD Calculation of Jet Cross-Sections in $e^{+} e^{-}$Annihilation, Nucl. Phys. B 187, 302 (1981)

145. R. K. Ellis and D. A. Ross, On the Thrust Distribution in $e^{+} e^{-}$Annihilation, Phys. Lett. B 106, 88 (1981)

146. Z. Kunszt, Magnitude of the $O\left(\alpha_{s}^{2}\right)$ Corrections to Jet Production in $e^{+} e^{-}$Annihilation, Phys. Lett. B 107, 123 (1981)

147. L. Clavelli and D. Wyler, Kinematical Bounds on Jet Variables and the Heavy Jet Mass Distribution, Phys. Lett. B 103, 383 (1981) 
148. A. Ali, The QCD Effective Coupling Constant in $e^{+} e^{-}$Annihilation, Phys. Lett. B 110, 67 (1982)

149. R. Brandelik et al. [TASSO Collaboration], Comparison of $e^{+} e^{-}$Annihilation with QCD and Determination of the Strong Coupling Constant, Phys. Lett. B 94, 437 (1980)

150. H. B. Newman, Results on Jets, QCD and Lepton Production from the Mark-J. (Talk), AIP Conf. Proc. 68, 627 (1981)

151. C. L. Basham, L. S. Brown, S. D. Ellis and S. T. Love, Energy Correlations in Electron - Positron Annihilation: Testing QCD, Phys. Rev. Lett. 41, 1585 (1978);

152. C. L. Basham, L. S. Brown, S. D. Ellis and S. T. Love, Energy Correlations in ElectronPositron Annihilation in Quantum Chromodynamics: Asymptotically Free Perturbation Theory, Phys. Rev. D 19, 2018 (1979)

153. A. Ali and F. Barreiro, An $O\left(\alpha_{s}^{2}\right)$ Calculation of Energy-Energy Correlation in $e^{+} e^{-}$ Annihilation and Comparison with Experimental Data, Phys. Lett. B 118, 155 (1982

154. A. Ali and F. Barreiro, Energy-Energy Correlations in $e^{+} e^{-}$Annihilation, Nucl. Phys. B 236, 269 (1984)

155. F. Csikor, Quark Mass Effects for Energy-Energy Correlations in High-Energy $e^{+} e^{-}$ Annihilation, Phys. Rev. D 30, 28 (1984)

156. K. H. Cho, S. K. Han and J. K. Kim, Energy-Energy Correlations in Electron Positron Annihilation: Z Boson and Heavy Quark Effects, Nucl. Phys. B 233, 161 (1984)

157. D. G. Richards, W. J. Stirling and S. D. Ellis, Second Order Corrections to the EnergyEnergy Correlation Function in Quantum Chromodynamics, Phys. Lett. B 119, 193 (1982)

158. D. G. Richards, W. J. Stirling and S. D. Ellis, Energy-Energy Correlations to Second Order in Quantum Chromodynamics, Nucl. Phys. B 229, 317 (1983)

159. W. Bartel et al. [JADE Collaboration], Measurements of Energy Correlations in $e^{+} e^{-} \rightarrow$ Hadrons, Z. Phys. C 25, 231 (1984)

160. B. Adeva et al. [MARK-J Collaboration], A Model Independent Second Order Determination of the Strong Coupling Constant $\alpha_{s}$, Phys. Rev. Lett. 50, 2051 (1983)

161. W. Braunschweig et al. [TASSO Collaboration], A Study Of Energy-Energy Correlations between $12 \mathrm{GeV}$ and $46.8 \mathrm{GeV}$ CM Energies, Z. Phys. C 36, 349 (1987)

162. C. Berger et al. [PLUTO Collaboration], A Study of Energy-Energy Correlations in $e^{+} e^{-}$Annihilations at $\sqrt{s}=34.6 \mathrm{GeV}$, Z. Phys. C 28, 365 (1985)

163. K. Fabricius, I. Schmitt, G. Schierholz and G. Kramer, Order $\alpha_{s}^{2}$ Correction to Jet Cross-Sections in $e^{+} e^{-}$Annihilation, Phys. Lett. B 97, 431 (1980)

164. K. Fabricius, I. Schmitt, G. Schierholz and G. Kramer, Higher Order Perturbative QCD Calculation of Jet Cross-Sections in $e^{+} e^{-}$Annihilation, Z. Phys. C 11, 315 (1981)

165. F. Gutbrod, G. Kramer and G. Schierholz, Higher Order QCD Corrections to the Three-Jet Cross-Sections: Bare Versus Dressed Jets, Z. Phys. C 21, 235 (1984)

166. G. Kramer and B. Lampe, Jet Cross-Sections in $e^{+} e^{-}$Annihilation, Fortsch. Phys. 37, 161 (1989)

167. F. Gutbrod, G. Kramer, G. Rudolph and G. Schierholz, Recombination Dependence of the $O\left(\alpha_{s}^{2}\right)$ Three-Jet Cross-Section in $e^{+} e^{-}$Annihilation, Z. Phys. C 35, 543 (1987)

168. S. Bethke et al. [JADE Collaboration], Experimental Investigation of the Energy Dependence of the Strong Coupling Strength, Phys. Lett. B 213, 235 (1988)

169. T. D. Gottschalk and M. P. Shatz, A Reassessment of the $O\left(\alpha_{s}^{2}\right)$ Three-Jet CrossSection for $e^{+} e^{-}$Annihilation, Phys. Lett. B 150, 451 (1985)

170. S. Komamiya et al., Determination of $\alpha_{s}$ from a Differential Jet Multiplicity Distribution at SLC and PEP, Phys. Rev. Lett. 64, 987 (1990)

171. W. A. Bardeen, A. J. Buras, D. W. Duke and T. Muta, Deep Inelastic Scattering Beyond the Leading Order in Asymptotically Free Gauge Theories, Phys. Rev. D 18, 3998 (1978)

172. R. Brock et al. [CTEQ Collaboration], Handbook of perturbative QCD: Version 1.0, Rev. Mod. Phys. 67, 157 (1995)

173. S. Bethke et al., Studies of Jet Production Rates in $e^{+} e^{-}$Annihilation at $\mathrm{E}(\mathrm{cm})=29$ GeV, Z. Phys. C 43, 325 (1989)

174. S. Bethke, An Experimental Approach to Optimize and Test Perturbative QCD to $O\left(\alpha_{s}^{2}\right)$, Z. Phys. C 43, $331(1989)$ 
175. I. H. Park et al. [AMY Collaboration], Experimental Evidence for the Non-Abelian Nature of QCD, Phys. Rev. Lett. 62, 1713 (1989)

176. W. Braunschweig et al. [TASSO Collaboration], Analysis of Multi-Jet Final States in $e^{+} e^{-}$Annihilation, Phys. Lett. B 214, 286 (1988)

177. B. Löhr et al., Variation of the Strong Coupling Constant from a Measurement of the Jet Energy Spread in $e^{+} e^{-}$Annihilation, Phys. Lett. B 122, 90 (1983)

178. H. J. Behrend et al. [CELLO Collaboration], Model Independent Limits on $\Lambda_{\mathrm{QCD}}$ from $e^{+} e^{-}$Annihilation in the Energy Range from $14 \mathrm{GeV}$ to $46 \mathrm{GeV}$, Z. Phys. C 44, 63 (1989) 179. W. Bartel et al. [JADE Collaboration], Experimental Study of Jets in Electron Positron Annihilation, Phys. Lett. B 101, 129 (1981)

180. W. Bartel et al. [JADE Collaboration], Test of Fragmentation Models by Comparison with Three-Jet Events Produced in $e^{+} e^{-} \rightarrow$ Hadrons, Phys. Lett. B 134, 275 (1984)

181. H. Aihara et al. [TPC/Two Gamma Collaboration], Tests of Models for Quark and Gluon Fragmentation in $e^{+} e^{-}$Annihilation at $\sqrt{s}=29 \mathrm{GeV}$, Z. Phys. C 28, 31 (1985)

182. M. Althoff et al. [TASSO Collaboration], A Study of Three-Jet Events in $e^{+} e^{-}$Annihilation into Hadrons at 34.6 GeV Center-of-Mass Energy, Z. Phys. C 29, 29 (1985)

183. P. D. Sheldon et al., A Comparison of the Particle Flow in Three-Jet and Radiative Two-Jet Events from $e^{+} e^{-}$Annihilation at $\mathrm{E}(\mathrm{cm})=29 \mathrm{GeV}$, Phys. Rev. Lett. 57, 1398 (1986)

184. G. Altarelli and G. Parisi, Asymptotic Freedom in Parton Language, Nucl. Phys. B 126, 298 (1977)

185. L. N. Lipatov, The Parton Model and Perturbation Theory, Sov. J. Nucl. Phys. 20, 94 (1975) [Yad. Fiz. 20, 181 (1974)]

186. V. N. Gribov and L. N. Lipatov, Deep Inelastic ep Scattering in Perturbation Theory, Sov. J. Nucl. Phys. 15, 438 (1972) [Yad. Fiz. 15, 781 (1972)]

187. Y. L. Dokshitzer, Calculation of the Structure Functions for Deep Inelastic Scattering and $e^{+} e^{-}$Annihilation by Perturbation Theory in Quantum Chromodynamics, Sov. Phys. JETP 46, 641 (1977) [Zh. Eksp. Teor. Fiz. 73, 1216 (1977)]

188. Y. I. Azimov, Y. L. Dokshitzer, V. A. Khoze and S. I. Troyan, Humpbacked QCD Plateau in Hadron Spectra, Z. Phys. C 31, 213 (1986)

189. P. Abreu et al. [DELPHI Collaboration], Measurement of the Gluon Fragmentation Function and a Comparison of the Scaling Violation in Gluon and Quark Jets, Eur. Phys. J. C 13, 573 (2000)

190. C. P. Fong and B. R. Webber, Higher Order QCD Corrections to Hadron Energy Distributions in Jets, Phys. Lett. B 229, 289 (1989)

191. M. Z. Akrawy et al. [OPAL Collaboration], A Study of Coherence of Soft Gluons in Hadron Jets, Phys. Lett. B 247, 617 (1990)

192. P. Abreu et al. [DELPHI Collaboration], The Scale-dependence of the Hadron Multiplicity in Quark and Gluon Jets and a Precise Determination of $C(A) / C(F)$, Phys. Lett. B 449, 383 (1999) arXiv:hep-ex/9903073.

193. I. M. Dremin and J. W. Gary, "Hadron multiplicities, Phys. Rept. 349, 301 (2001) arXiv:hep-ph/0004215

194. Y. L. Dokshitzer, V. A. Khoze and S. I. Troian, Specific Features of Heavy Quark Production. 1. Leading Quarks, Leningrad Report LU-TP-92-10 (1992)

195. C. Pahl, S. Bethke, S. Kluth, J. Schieck and the Jade collaboration, Study of Moments of Event Shapes and a Determination of $\alpha_{s}$ using $e^{+} e^{-}$Annihilation Data from Jade, Eur. Phys. J. C 60, 181 (2009) [Erratum-ibid. C 62, 451 (2009)] arXiv:0810.2933 [hep-ex]]

196. P. Zerwas, W \& Z Physics at LEP, Eur. Phys. J. C 34, 41 (2004) and in Prestigious discoveries at CERN, ed. R. Cashmore, G. Maiani and J.-P. Revol, Springer Verlag, (2004)

197. P. Abreu et al. [DELPHI Collaboration], Energy-dependence of Event Shapes and of $\alpha_{s}$ at LEP2, Phys. Lett. B 456, 322 (1999)

198. P. Pfeifenschneider et al. [JADE collaboration and OPAL Collaboration], QCD Analyses and Determinations of $\alpha_{s}$ in $e^{+} e^{-}$Annihilation at Energies between $35 \mathrm{GeV}$ and 189 GeV, Eur. Phys. J. C 17, 19 (2000)

199. P. Achard et al. [L3 Collaboration], Determination of $\alpha_{s}$ from Hadronic Event Shapes in $e^{+} e^{-}$Annihilation at $192 \mathrm{GeV} \leq \sqrt{s} \leq 208 \mathrm{GeV}$, Phys. Lett. B 536, 217 (2002) 
200. A. Heister et al. [ALEPH Collaboration], Studies of QCD at $e^{+} e^{-}$Centre-of-Mass Energies between $91 \mathrm{GeV}$ and $209 \mathrm{GeV}$, Eur. Phys. J. C 35, 457 (2004)

201. S. Catani, G. Turnock, B. R. Webber and L. Trentadue, Thrust Distribution in $e^{+} e^{-}$ Annihilation," Phys. Lett. B 263, 491 (1991)

202. S. Catani, G. Turnock and B. R. Webber, Heavy Jet Mass Distribution in $e^{+} e^{-}$annihilation," Phys. Lett. B 272, 368 (1991)

203. S. Catani, L. Trentadue, G. Turnock and B. R. Webber, Resummation of Large Logarithms in $e^{+} e^{-}$Event Shape Distributions, Nucl. Phys. B 407, 3 (1993)

204. L. Lönnblad, Ariadne Version 4: A Program for Simulation of QCD Cascades Implementing the Color Dipole Model, Comput. Phys. Commun. 71, 15 (1992)

205. Y. L. Dokshitzer and B. R. Webber, Calculation of Power Corrections to Hadronic Event Shapes, Phys. Lett. B 352, 451 (1995) arXiv:hep-ph/9504219.

206. Y. L. Dokshitzer, A. Lucenti, G. Marchesini and G. P. Salam, Universality of 1/Q Corrections to Jet-shape Observables Rescued, Nucl. Phys. B 511, 396 (1998) [Erratumibid. B 593, 729 (2001)]

207. Y. L. Dokshitzer, A. Lucenti, G. Marchesini and G. P. Salam, On the Universality of the Milan Factor for 1/Q Power Corrections to Jet Shapes, JHEP 9805, 003 (1998)

208. M. Dasgupta and G. P. Salam, Event shapes in e+ e- annihilation and deep inelastic scattering, J. Phys. G 30, R143 (2004) arXiv:hep-ph/0312283

209. G. Dissertori, A. Gehrmann-De Ridder, T. Gehrmann, E. W. N. Glover, G. Heinrich and H. Stenzel, Precise Determination of the Strong Coupling Constant at NNLO in QCD from the Three-Jet Rate in Electron-Positron Annihilation at LEP," Phys. Rev. Lett. 104, $072002(2010)$

210. A. Signer and L. J. Dixon, Electron Positron Annihilation into Four Jets at Next-toLeading Order in $\alpha_{s}$, Phys. Rev. Lett. 78, 811 (1997)

211. L. J. Dixon and A. Signer, Complete $O\left(\alpha_{s}^{3}\right)$ Results for $e^{+} e^{-} \rightarrow(\gamma, Z) \rightarrow$ Four Jets, Phys. Rev. D 56, 4031 (1997)

212. Z. Nagy and Z. Trocsanyi, Next-to-Leading Order Calculation of Four-Jet Observables in Electron Positron Annihilation, Phys. Rev. D 59, 014020 (1999) [Erratum-ibid. D 62, $099902(2000)]$

213. P. D. Acton et al. [OPAL Collaboration], A Global Determination of $\alpha_{s}\left(M_{Z}\right)$ at LEP, Z. Phys. C 55, 1 (1992)

214. R. Frederix, S. Frixione, K. Melnikov and G. Zanderighi, NLO QCD Corrections to Five-Jet Production at LEP and the Extraction of $\alpha_{s}\left(M_{Z}\right)$, JHEP 11:050 (2010)

215. T. Sjostrand, S. Mrenna and P. Z. Skands, PYTHIA 6.4 Physics and Manual, JHEP 0605, 026 (2006)

216. G. Corcella et al., HERWIG 6.5: An Event Generator for Hadron Emission Reactions with Interfering Gluons (including Supersymmetric Processes), JHEP 0101, 010 (2001)

217. S. Catani, F. Krauss, R. Kuhn and B. R. Webber, QCD Matrix Elements + Parton Showers, JHEP 0111, 063 (2001)

218. M. Bengtsson and P. M. Zerwas, Four-Jet Events in $e^{+} e^{-}$Annihilation: Testing the Three-Gluon Vertex, Phys. Lett. B 208, 306 (1988)

219. O. Nachtmann and A. Reiter, A Test for the Gluon Self Coupling in the Reactions $e^{+} e^{-} \rightarrow$ Four Jets and $Z \rightarrow$ Four Jets, Z. Phys. C 16, 45 (1982)

220. J. G. Körner, G. Schierholz and J. Willrodt, QCD Predictions for Four-Jet Final States in $e^{+} e^{-}$Annihilation: 2. Angular Correlations as a Test of the Triple Gluon Coupling, Nucl. Phys. B 185, 365 (1981)

221. Z. Nagy and Z. Trocsanyi, Four-Jet Angular Distributions and Color Charge Measurements: Leading Order versus Next-to-Leading Order, Phys. Rev. D 57, 5793 (1998) arXiv:hep-ph/9712385.

222. B. Adeva et al. [L3 Collaboration], A Test of QCD Based on Four-Jet Events from $Z$ Decays, Phys. Lett. B 248, 227 (1990)

223. G. Abbiendi et al. [OPAL Collaboration], A Simultaneous Measurement of the QCD Colour Factors and the Strong Coupling, Eur. Phys. J. C 20, 601 (2001)

224. M. Czakon, The Four-Loop QCD Beta-function and Anomalous Dimensions, Nucl. Phys. B 710, 485 (2005) 
225. T. van Ritbergen, J. A. M. Vermaseren and S. A. Larin, The Four-Loop Beta Function in Quantum Chromodynamics, Phys. Lett. B 400, 379 (1997)

226. T. Gehrmann, M. Jaquier and G. Luisoni, Hadronization Effects in Event Shape Moments, Eur. Phys. J. C 67,57 (2010)

227. G. Dissertori, A. Gehrmann-De Ridder, T. Gehrmann, E. W. N. Glover, G. Heinrich, G. Luisoni and H. Stenzel, Determination of the Strong Coupling Constant Using Matched NNLO+NLLA Predictions for Hadronic Event Shapes in $e^{+} e^{-}$Annihilations, JHEP 0908, $036(2009)$

228. S. Bethke, The 2009 Wolrd Average of $\alpha_{s}\left(M_{Z}\right)$, Eur. Phys. J. C 64, 689 (2009)

229. J. C. Collins, D. E. Soper, G. F. Sterman, Adv. Ser. Direct. High Energy Phys. 5, 1-91 (1988) hep-ph/0409313

230. We follow here the review by G. Dissertori and G.P. Salam on "Quantum Chromodynamics" in Ref. 7 .

231. P.M. Nadolsky et al., Implications of CTEQ Global Analysis for Collider Observables, Phys. Rev. D 78, 013004 (2008), and earlier references given therein

232. A.D. Martin et al., Parton Distributions for the LHC, Eur. Phys. J. C 63, 189 (2009), and earlier papers referenced therein

233. S. Catani and M.H. Seymour, A General Algorithm for Calculating Jet Cross-Sections in NLO QCD, Nucl. Phys. B 485, 291 (1997); [Erratum: Nucl. Phys. B 510, 503 (1998)]

234. Z. Nagy and Z. Troscanyi, Multijet Cross-Sections in Deep Inelastic Scattering at Nextto-Leading Order, Phys. Rev. Lett. 87, 082001 (2001)

235. C. Amsler et al. (Particle Data Group), Review of Particle Physics, Phys. Lett. B 667, 1 (2008)

236. F.D. Aaron et al. [H1 Collaboratio], Jet Production in ep Collisions at High $Q^{2}$ and Determination of $\alpha_{s}$, Eur. Phys. J. C 65, 363 (2010)

237. S. Chekanov et al. [ZEUS Collaboration], Inclusive Jet Cross Sections in the Breit frame in Neutral Current Deep Inelastic Scattering at HERA and Determination of $\alpha_{s}$, Phys. Lett. B 547, 164 (2002)

238. S. Chekanov et al. [ZEUS Collaboration], Inclusive-Jet and Dijet Cross Sections in Deep Inelastic Scattering at HERA, Nucl. Phys. B 765, 1 (2007)

239. A. Aktas et al. [H1 Collaboration], Measurement of Inclusive Jet Production in DeepInelastic Scattering at High $Q^{2}$ and Determination of the Strong Coupling, Phys. Lett. B 653, 134 (2007)

240. S. Chekanov et al. [ZEUS Collaboration], Jet-Radius Dependence of Inclusive-Jet Cross-Sections in Deep Inelastic Scattering at HERA, Phys. Lett. B 649, 12 (2007)

241. C. F. von Weizsäcker, Radiation Emitted in Collisions of Very Fast Electrons (in German), Z. Phys. 88, 612 (1934)

242. E.J. Williams, Nature of the High-Energy Particles of Penetrating Radiation and Status of Ionization and Radiation Formulae, Phys. Rev. 45, 729 (1934)

243. S. Frixione et al., Improving the Weizsacker-Williams Approximation in Electron Proton Collisions, Phys. Lett. B 319, 339 (1993)

244. C. H. Llewellyn Smith, QCD Predictions for Processes Involving Real Photons, Phys. Lett. B 79, 83 (1978)

245. S. J. Brodsky, T. A. DeGrand, J. F. Gunion and J. H. Weis, Production of Large Transverse Momentum Jets in Photon - Photon Collisions, Phys. Rev. Lett. 41, 672 (1978)

246. M. Klasen and G. Kramer, Inclusive Two-Jet Production at HERA: Direct and Resolved Cross-Sections in Next-to-Leading Order QCD, Z. Phys. C 76, 67 (1997

247. M. Klasen, T. Kleinwort and G. Kramer, Inclusive Jet Production in $\gamma p$ and $\gamma \gamma$ Processes: Direct and Resolved Photon Cross-Sections in Next-to-Leading Order QCD, Eur. Phys. J. direct C 1, 1 (1998)

248. B.W. Harris and J.F. Owen, Jet Photoproduction and the Structure of the Photon, Phys. Rev. D 57, 5555 (1998)

249. S. Frixione and G. Ridolfi, Jet Photoproduction at HERA, Nucl. Phys. B507, 315 (1997)

250. A. Aktas et al. [H1 Collaboration], Photoproduction of Dijets with High Transverse Momenta at HERA, Phys. Lett. B 639, 21 (2006) 
251. S. Chekanov et al. [ZEUS Collaboration], High- $E_{T}$ Dijet Photoproduction at HERA, Phys. Rev. D 76, 072011 (2007)

252. S. Chekanov et al. [ZEUS Collaboration], High Mass Dijet Cross-Sections in Photoproduction at HERA, Phys. Lett. B 531, 9 (2002)

253. S. Chekanov et al. [ZEUS Collaboration], Dijet Photoproduction at HERA and the Structure of the Photon, Eur. Phys. J. C 23, 615 (2002)

254. P. Aurenche, M. Fontannaz and J. P. Guillet, New NLO Parametrizations of the Parton Distributions in Real Photons, Eur. Phys. J. C 44, 395 (2005)

255. F. Cornet, P. Jankowski and M. Krawczyk, A New 5 Flavour NLO Analysis and Parametrizations of Parton Distributions of the Real Photon, Phys. Rev. D 70, 093004 (2004)

256. S. Chekanov et al. [ZEUS Collaboration], Scaling Violations and Determination of $\alpha_{s}$ from Jet Production in $\gamma p$ Interactions at HERA, Phys. Lett. B 560, 7 (2003)

257. P. Aurenche, J. P. Guillet and M. Fontannaz, Parton Distributions in the Photon, Z. Phys. C 64, 621 (1994)

258. M. Glück, E. Reya and A. Vogt, Parton Structure of the Photon Beyond the Leading Order, Phys. Rev. D 45, 3896 (1992)

259. M. Gluck, E. Reya and A. Vogt, Photonic Parton Distributions, Phys. Rev. D 46, 1973 (1992)

260. W. Slominski, H. Abramowicz and A. Levy, NLO Photon Parton Parametrization Using $e^{+} e^{-}$and $e p$ Data, Eur. Phys. J. C 45, 633 (2006)

261. V. S. Fadin, E. A. Kuraev and L. N. Lipatov, On the Pomeranchuk Singularity in Asymptotically Free Theories, Phys. Lett. B 60, 50 (1975)

262. I. I. Balitsky and L. N. Lipatov, The Pomeranchuk Singularity in Quantum Chromodynamics, Sov. J. Nucl. Phys. 28, 822 (1978) [Yad. Fiz. 28, 1597 (1978)]

263. M. Dittmar et al., Summary Report for the HERA - LHC Workshop Proceedings: Parton Distributions, Report FUM-936-FT, arXiv:0901.2504 [hep-ph] (2009)

264. L. McLerran, Gluon Evolution and Saturation Proceedings, presented at the Gribov Memorial Workshop on Quantum Chromodynamics and Beyond, arXiv:1011.3202 [hep$\mathrm{ph}]](2010)$

265. For the concept and definitions of the terms Color Glass Condensate, see, L. McLerran, The CGC and the Glasma: Two Lectures at the Yukawa Insitute, arXiv:1011.3204 [hep$\mathrm{ph}] \mathrm{(2010)}$

266. See, for a review, E. Iancu and R. Venugopalan, The Color Glass Condensate and High Energy Scattering in QCD, in Quark gluon plasma, edited by R. Hwa and X.N. Wang, World Scientific, 2003, arXiv:hep-ph/0303204 (2003)

267. S. J. Brodsky, F. E. Close and J. F. Gunion, Phenomenology of Photon Processes, Vector Dominance and Crucial Tests for Parton Models, Phys. Rev. D6, 177 (1972)

268. J. Kwiecinski, B. M. Badelek, Analysis of the Electroproduction Structure Functions in the Low $\mathrm{Q}^{* *} 2$ Region combining the Vector Meson Dominance and the Parton Model with possible Scaling Violation," Z. Phys. C43, 251 (1989)

269. G. Abbiendi et al. [OPAL Collaboration], Di-jet Production in Photon Photon Collisions at $\sqrt{s}_{e e}=$ from $189 \mathrm{GeV}$ to $209 \mathrm{GeV}$, Eur. Phys. J. C 31, 307 (2003)

270. J. Abdallah et al. [DELPHI Collaboration], Di-jet Production in Gamma-Gamma Collisions at LEP2, Eur. Phys. J. C 58, 531 (2008)

271. P. Achard et al. [L3 Collaboration], Inclusive Jet Production in Two-Photon Collisions at LEP, Phys. Lett. B 602, 157 (2004)

272. G. Abbiendi et al. [OPAL Collaboration], Inclusive Jet Production in Photon-Photon Collisions at $\sqrt{s}_{e e}=$ from 189 to $209 \mathrm{GeV}$, Phys. Lett. B 658, 185 (2008)

273. V. M. Abazov et al. [D0 Collaboration], Measurement of the Inclusive Jet Cross-Section in $p \bar{p}$ Collisions at $\sqrt{s}=1.96 \mathrm{TeV}$, Phys. Rev. Lett. 101, 062001 (2008)

274. Measurement of the Inclusive Jet Cross Section in $p p$ Collisions at $7 \mathrm{TeV}, \mathrm{CMS}$ Physics Analysis Summary, CMS PAS QCD-10-011 (2010)

275. A. Bhatti and D. Lincoln, Jet Physics at the Tevatron, FERMILAB-PUB-10-028-EPPD [arXiv:1002.1708 [hep-ex]] (2010) 
276. T. Aaltonen et al. [CDF Collaboration], Measurement of the Inclusive Jet Cross Section at the Fermilab Tevatron $p \bar{p}$ Collider Using a Cone-Based Jet Algorithm, Phys. Rev. D 78, 052006 (2008) [Erratum-ibid. D 79, 119902 (2009]

277. A. Abulencia et al. [CDF - Run II Collaboration], Measurement of the Inclusive Jet Cross Section Using the $\boldsymbol{k}_{\mathrm{T}}$ Algorithm in $\boldsymbol{p} \overline{\boldsymbol{p}}$ Collisions at $\sqrt{\boldsymbol{s}}=1.96 \mathrm{TeV}$ with the CDF II Detector, Phys. Rev. D 75, 092006 (2007) [Erratum-ibid. D 75, 119901 (2007)]

278. S. D. Ellis, J. Huston, K. Hatakeyama, P. Loch and M. Tonnesmann, Jets in hadronhadron collisions, Prog. Part. Nucl. Phys. 60, 484 (2008) arXiv:0712.2447 [hep-ph]]

279. Z. Nagy, Three-jet Cross Sections in Hadron Hadron Collisions at Next-to-leading Order, Phys. Rev. Lett. 88, 122003 (2002)

280. M. Bähr et al., Herwig++ Physics and Manual, Eur. Phys. J. C 58, 639 (2008)

281. A. Buckley et al., arXiv:1101.2599 [hep-ph].

282. E. Eichten, K. D. Lane and M. E. Peskin, New Tests for Quark and Lepton Substructure, Phys. Rev. Lett. 50, 811 (1983)

283. U. Baur, I. Hinchliffe and D. Zeppenfeld, Excited Quark Production at Hadron Colliders, Int. J. Mod. Phys. A 2, 1285 (1987)

284. U. Baur, M. Spira and P. M. Zerwas, Excited Quark and Lepton Production at Hadron Colliders, Phys. Rev. D 42, 815 (1990)

285. P. H. Frampton and S. L. Glashow, Chiral Color: An Alternative to the Standard Model, Phys. Lett. B 190, 157 (1987)

286. J. Bagger, C. Schmidt and S. King, Axigluon Production in Hadronic Collisions, Phys. Rev. D 37, 1188 (1988)

287. R. S. Chivukula, A. G. Cohen and E. H. Simmons, New Strong Interactions at the Tevatron?, Phys. Lett. B 380, 92 (1996)

288. E. H. Simmons, Coloron phenomenology, Phys. Rev. D 55, 1678 (1997)

289. K. D. Lane and M. V. Ramana, Walking Technicolor Signatures at Hadron Colliders, Phys. Rev. D 44, 2678 (1991)

290. K. Lane and S. Mrenna, The Collider Phenomenology of Technihadrons in the Technicolor Straw Man Model, Phys. Rev. D 67, 115011 (2003)

291. R. Foadi, M. T. Frandsen, T. A. Ryttov and F. Sannino, Minimal Walking Technicolor: Set Up for Collider Physics, Phys. Rev. D 76, 055005 (2007)

292. A. Belyaev et al., Technicolor Walks at the LHC, Phys. Rev., D 79, 035006 (2009)

293. T. Aaltonen et al. [CDF Collaboration], Search for New Particles Decaying into Dijets in Proton-Antiproton Collisions at $\sqrt{s}=1.96 \mathrm{TeV}$, Phys. Rev. D 79, 112002 (2009)

294. G. Aad et al. [ATLAS Collaboration], Search for New Particles in Two-Jet Final States in $7 \mathrm{TeV}$ Proton-Proton Collisions with the ATLAS Detector at the LHC, Phys. Rev. Lett. 105, $161801(2010)$

295. V. Khachatryan et al. [CMS Collaboration], Search for Dijet Resonances in $7 \mathrm{TeV}$ pp Collisions at CMS, Phys. Rev. Lett. 105, 211801 (2010)

296. R. K. Ellis, G. Martinelli and R. Petronzio, Lepton Pair Production at Large Transverse Momentum in Second Order QCD, Nucl. Phys. B 211, 106 (1983)

297. P. B. Arnold and M. H. Reno, The Complete Computation of High $p_{t} W$ and $Z$ Production in 2nd Order QCD, Nucl. Phys. B 319, 37 (1989) [Erratum-ibid. B 330, 284 (1990)]

298. P. B. Arnold, R. K. Ellis and M. H. Reno, High $p_{t} W$ and $Z$ Production at the Tevatron, Phys. Rev. D 40, 912 (1989)

299. W. T. Giele, E. W. N. Glover and D. A. Kosower, Higher Order Corrections to Jet Cross-Sections in Hadron Colliders, Nucl. Phys. B 403, 633 (1993)

300. J. M. Campbell and R. K. Ellis, Next-to-Leading Order Corrections to $W+2$ Jet and $Z+$ 2Jet Production at Hadron Colliders, Phys. Rev. D 65, 113007 (2002)

301. J. M. Campbell, R. K. Ellis and D. L. Rainwater, Next-to-Leading Order QCD Predictions for $W+$ 2Jet and $Z+$ 2Jet Production at the CERN LHC, Phys. Rev. D 68, 094021 (2003)

302. F. A. Berends, W. T. Giele, H. Kuijf, R. Kleiss and W. J. Stirling, Multi - Jet Production in $W, Z$ Events at $p \bar{p}$ Colliders, Phys. Lett. B 224, 237 (1989) 
303. F. A. Berends, H. Kuijf, B. Tausk and W. T. Giele, On the Production of a $W$ and Jets at Hadron Colliders, Nucl. Phys. B 357, 32 (1991)

304. J. M. Campbell and R. K. Ellis, MCFM for the Tevatron and the LHC, Nucl. Phys. Proc. Suppl. 205-206, 10 (2010); MCFM homepage, http://mcfm.fnal.gov

305. K. Melnikov and F. Petriello, Electroweak Gauge Boson Production at Hadron Colliders through $O\left(\alpha_{s}^{2}\right)$, Phys. Rev. D 74, 114017 (2006)

306. S. Catani, L. Cieri, G. Ferrera, D. de Florian and M. Grazzini, Vector Boson Production at Hadron Colliders: A Fully Exclusive QCD Calculation at NNLO, Phys. Rev. Lett. 103, 082001 (2009)

307. G. A. Ladinsky and C. P. Yuan, The Nonperturbative Regime in QCD Resummation for Gauge Boson Production at Hadron Colliders, Phys. Rev. D 50, 4239 (1994)

308. C. Balazs and C. P. Yuan, Soft Gluon Effects on Lepton Pairs at Hadron Colliders, Phys. Rev. D 56, 5558 (1997)

309. V. M. Abazov et al. [D0 Collaboration], Measurement of the Normalized $Z / \gamma^{*} \rightarrow \mu^{+} \mu^{-}$ Transverse Momentum Distribution in $p \bar{p}$ Collisions at $\sqrt{s}=1.96 \mathrm{TeV}$, Phys. Lett. B 693, $522(2010)$

310. Measurement of $Z / \gamma^{*} \rightarrow \mu^{+} \mu^{-}+$Jets Production Cross Section, CDF Note 10216, July 10, 2010 (CDF Collaboration); URL: http://www-cdf-fnal.gov

311. For a recent discussion of the standard electroweak model, see the review by J. Erler and P. Langacker: "Electroweak model and constraints on new physics" in Ref. [7.

312. D. E. Acosta et al. [CDF Collaboration], Measurement of the $W^{+} W^{-}$Production Cross Section in $p \bar{p}$ Collisions at $\sqrt{s}=1.96 \mathrm{TeV}$ using Dilepton Events, Phys. Rev. Lett. 94, $211801(2005)$

313. T. Aaltonen et al. [CDF Collaboration], First Measurement of $Z Z$ Production in $p \bar{p}$ Collisions at $\sqrt{s}=1.96 \mathrm{TeV}$, ibid 100, 201801 (2008)

314. V. M. Abazov et al. [D0 Collaboration], Measurement of the $W W$ Production Cross Section in $p \bar{p}$ Collisions at $\sqrt{s}=1.96 \mathrm{TeV}$, Phys. Rev. Lett. 94, 151801 (2005) [Erratumibid. 100, 139901 (2008)]

315. V. M. Abazov et al. [D0 Collaboration], Evidence of $W W+W Z$ Production with Lepton + Jets Final States in Proton-Antiproton Collisions at $\sqrt{s}=1.96 \mathrm{TeV}$, Phys. Rev. Lett. 102, 161801 (2009)

316. T. Aaltonen et al. [CDF Collaboration], First Observation of Vector Boson Pairs in a Hadronic Final State at the Tevatron Collider, Phys. Rev. Lett. 103, 091803 (2009)

317. J. M. Campbell and R. K. Ellis, An Update on Vector Boson Pair Production at Hadron Colliders, Phys. Rev. D 60, 113006 (1999)

318. T. Aaltonen et al. [The CDF Collaboration], Measurement of the Top Quark Mass and $p \bar{p} \rightarrow t \bar{t}$ Cross Section in the All-Hadronic Mode with the CDFII Detector, Phys. Rev. D 81, $052011(2010)$

319. A. Djouadi, M. Spira and P. M. Zerwas, QCD Corrections to Hadronic Higgs Decays, Z. Phys. C 70, 427 (1996)

320. J. M. Butterworth, A. R. Davison, M. Rubin and G. P. Salam, Jet Substructure as a New Higgs Search Channel at the LHC, Phys. Rev. Lett. 100 (2008) 242001

321. T. Aaltonen et al. [CDF Collaboration], Search for Standard Model Higgs Boson Production in Association with a $W$ Boson at CDF, Phys. Rev. D 78, 032008 (2008)

322. T. Aaltonen et al. [The CDF Collaboration], Search for Doubly Charged Higgs Bosons with Lepton-Flavor-Violating Decays involving Tau Leptons, Phys. Rev. Lett. 101, 121801 (2008)

323. T. Aaltonen et al. [CDF Collaboration], A Search for the Associated Production of the Standard-Model Higgs Boson in the All-Hadronic Channel, Phys. Rev. Lett. 103, 221801 (2009)

324. T. Plehn, G. P. Salam and M. Spannowsky, Fat Jets for a Light Higgs, Phys. Rev. Lett. 104, $111801(2010)$

325. J. M. Butterworth, J. R. Ellis and A. R. Raklev, Reconstructing Sparticle Mass Spectra using Hadronic Decays, JHEP 0705 (2007) 033

326. J. M. Butterworth, B. E. Cox and J. R. Forshaw, $W W$ Scattering at the CERN LHC, Phys. Rev. D 65, 096014 (2002) 
327. U. Baur and L. H. Orr, Searching for $t \bar{t}$ Resonances at the Large Hadron Collider, Phys. Rev. D 77, 114001 (2008)

328. S. Moch, J. A. M. Vermaseren and A. Vogt, The Three-loop Splitting Functions in QCD: The Non-Singlet Case, Nucl. Phys. B 688, 101 (2004)

329. A. Vogt, S. Moch and J. A. M. Vermaseren, The Three-loop Splitting Functions in QCD: The Singlet Case," Nucl. Phys. B 691, 129 (2004) 